Universidade de São Paulo

Instituto de Física

\title{
Resposta não linear do vácuo a distribuições de correntes na ausência de um campo externo de fundo e suas aplicações a pulsares e estrelas de quarks
}

\author{
Caio Vinícius Costa Lopes
}

Dissertação de mestrado apresentada ao

Instituto de Física para a obtenção do título de Mestre em Ciências

Orientador: Prof. Dr. Dimitri Maximovitch Gitman

Banca Examinadora:

Prof. Dr. Josif Frenkel (IF-USP)

Prof. Dr. Paulo Teotônio Sobrinho (IF-USP)

Prof. Dr. José Abdalla Helayel-Neto (CBPF) 


\section{Resumo}

Nesta dissertação de mestrado, nós encontramos a correção não linear aos campos elétrico e magnético produzidos por distribuições de cargas estacionárias, como o monopolo elétrico e o dipolo magnético homogeneamente polarizado em repouso, no vácuo, ou seja, imersas em um campo externo de fundo nulo. A função de resposta não linear é dada pelo tensor de polarização de quarta ordem, devido ao espalhamento de dois fótons, e é escrita dentro da aproximação da ação efetiva em eletrodinâmica quântica. Como aplicações realizamos estimativas numéricas para a correção dos campos produzidos em pulsares e estrelas de quarks, e concluímos quão relevantes são tais correções. Além disto, justificamos a divergência da autoenergia de uma carga eletrostática puntiforme (catástrofe ultravioleta) através do efeito de não linearidade. 


\begin{abstract}
On this master's dissertation, we find a nonlinear correction to the electric and magnetic fields produced by stationary charge distributions, like the electric monopole and the magnetic dipole uniformly polarized, at rest, in the vacuum, that is, it is immersed in a null external background field. The nonlinear response function is given by the fourth rank polarization tensor due to the scattering of two photons, and it is written within the approximation of effective action in quantum electrodynamics. As applications, numerical estimates are evaluated for the correction of the fields produced in quark stars and pulsars, and we conclude how relevant are such corrections. Furthermore, we explaned the self-energy's divergence of a puntiform electrostatic charge (ultraviolet catastroph) through the nonlinear effect.
\end{abstract}




\section{Agradecimentos}

Ao meu orientador Professor Dr. Dmitri M. Gitman e a todos os professores do Instituto de Física da USP que contribuíram para a minha formação.

Ao Professor Dr. Anatoly E. Shabad, colaborador do Grupo Quanta, por seu papel crucial no desenvolvimento do tema abordado.

À minha mãe Sra. Celia Regina Maria Costa Lopes, ao meu pai Sr. Carlos Roberto Lopes e minha tia Sra. Marlene Costa Gallo pelo incentivo e apoio.

Ao Tiago Carlos Adorno, João Luis Meloni Assirati e Rodrigo Fresneda pela amizade e discussões técnicas.

Aos amigos Edmilson Macedo (in memoriam), Marcelo Meireles dos Santos, Alberto Pereira, Maxim Dvornikov, Ignat Fialkovskiy, e aos inúmeros amigos no IFUSP e outros institutos.

A Deus, sobretudo, que me capacitou para realizar essa dissertação, por um propósito de vida, e a Ele seja a glória. 
$A$ realidade está bem distante $e$ é muito profunda; quem pode descobri-la?

Por isso dediquei-me a aprender, a investigar, a buscar a sabedoria e a razão de ser das coisas, para compreender a insensatez da impiedade e a loucura da insensatez. Eclesiastes 7:24-25 
Dedico este trabalho a Deus e seu Reino. 


\section{Sumário}

1 Notações $r$

2 Introdução $r$

3 Eletrodinâmica não linear em um campo de fundo constante 13

4 Efeito magneto-elétrico $\quad 16$

4.1 Aproximação infravermelho . . . . . . . . . . . . . . . . . . . 16

4.2 Campo magnético de uma carga estática em um campo magnético externo de fundo . 17

4.3 Lei de Coulomb anisotrópica . . . . . . . . . . . . . . . . . . . . . . . . . . 19

4.4 Diagrama de três fótons . . . . . . . . . . . . . . . . . . . . . . 20

5 Problemas estacionários em um campo de fundo nulo 21

5.1 Resposta linear e tensor de polarização de terceira ordem . . . . . . . . . . . . . . . . 21

5.2 Corrente não linearmente induzida . . . . . . . . . . . . . . . . . . . . . 22

6 Equações de Maxwell não lineares perturbativas e estacionárias 24

6.1 Soluções das equações inomogêneas . . . . . . . . . . . . . . . . . . . . . . . . 25

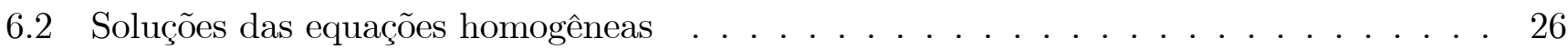

6.3 Magnetização, polarização e campos auxiliares não lineares . . . . . . . . . . . . . . 27

7 Aplicações a correntes estacionárias $r$

7.1 Correções para monopolos eletrostáticos . . . . . . . . . . . . . . . . . . . . . . 29

7.1.1 Simetria esférica: A esfera carregada . . . . . . . . . . . . . . . 29

7.1.2 Simetria cilíndrica com translação: O plano, o fio e a espira infinitos, e carregados 30

7.2 Correções para dipolos eletrostáticos e magnetostáticos . . . . . . . . . . . . . . . 32

7.2.1 Simetria cilíndrica: A esfera uniformemente magnetizada . . . . . . . . . . . . 32

7.2.1.1 Campo magnético produzido por pulsares de Rádio, Raios-X, e Mag-

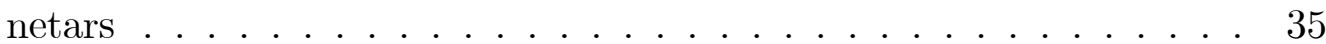

7.2.1.2 Momento magnético anômalo dos Bárions $\Xi^{* 0}, \Delta^{0}$ e $\Sigma^{* 0} \ldots \ldots 35$

7.2.2 Simetria cilíndrica: A esfera uniformemente polarizada . . . . . . . . . . 35

8 Modelos estacionários de solução exata e a auto-energia do elétron 37

8.1 O modelo de Born-Infeld . . . . . . . . . . . . . . . . . . . . . . . 37

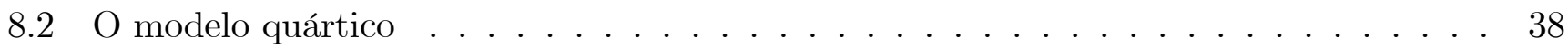

8.2.1 Equações para os campos . . . . . . . . . . . . . . . . . 39

8.2.2 Uma solução exata para o modelo quártico . . . . . . . . . . . . . . . . . . 40

8.2.3 Soluções assintóticas . . . . . . . . . . . . . . . . . . 40 
8.2.3.1 Solução perturbativa . . . . . . . . . . . . . . . . . . . 40

8.2.3.2 Solução para campos fortes . . . . . . . . . . . . . . 41

8.2.4 Finitude da energia do campo produzido por uma carga puntiforme . . . . . . 42

8.2.5 Cálculo da massa do campo elétrico em QED . . . . . . . . . . . . . . . . 43

8.3 O modelo polinomial . . . . . . . . . . . . . . . . . . . . . . . 43

9 Conclusão $\quad 45$

$\begin{array}{lr}\text { A Derivadas variacionais } & \mathbf{4 7}\end{array}$

A.1 Séries de Taylor variacionais e resolução das equações de Euler-Lagrange perturbativas 47

A.2 Derivadas variacionais da ação efetiva . . . . . . . . . . . . . . . . . . . . 48

A.2.1 Primeira ordem . . . . . . . . . . . . . . . . . . . . . . 48

A.2.2 Segunda ordem . . . . . . . . . . . . . . . . . . . . . . . . . . . . . . . . . . . . . . . . 49

A.2.3 Terceira ordem . . . . . . . . . . . . . . . . . . . . . . . . . . . . . . . . . 49

A.2.4 Quarta ordem . . . . . . . . . . . . . . . . . 49

B Existência das soluções das equações de Maxwell extacionárias: Os operadores de projeção

B.1 Operador de projeção longitudinal . . . . . . . . . . . . . . . . . . 51

B.2 Operador de projeção transversal . . . . . . . . . . . . . . . . . . . 52

C Unicidade das soluções das equações de Maxwell estacionárias $\quad 54$

D Solução de equações cúbicas: A fórmula de Tartaglia-Cardano 55 


\section{Capítulo 1}

\section{Notações}

Coordenadas do espaço de Minkowski são denotadas por:

$$
x=\left(x^{\mu}\right)=\left(x^{0}, x^{i}\right), \mu=0,1,2,3, i=1,2,3 .
$$

O sistema de unidades utilizado é tal que $c=\hbar=\varepsilon_{0}=1, e=\sqrt{4 \pi \alpha}$, onde $c$ é a velocidade da luz no vácuo, $\hbar$ é a constante de Planck reduzida, $e$ é a carga elementar do elétron, $\varepsilon_{0}$ é a permissividade do vácuo, e $\alpha$ é a constante de estrutura fina.

Assim, as coordenadas espaço-temporais são denotadas por:

$$
x^{0}=t, \mathbf{r}=\left(x^{i}\right)=\left(x^{1}, x^{2}, x^{3}\right), x=(t, \mathbf{r}) .
$$

Índices gregos em notação tensorial assumem os valores 0,1,2,3 e índices latinos 1,2,3 A métrica no espaço-tempo é determinada pelos tensores de Minkowski:

$$
\eta_{\mu \nu}=\operatorname{diag}(1,-1,-1,-1), \eta_{\mu \alpha} \eta^{\alpha \nu}=\delta_{\mu}^{\nu} .
$$

Via $\epsilon^{\mu \nu \alpha \beta}$, denotamos o tensor completamente anti-simétrico de Levi-Civita em $3+1$ dimensões com a normalização $\epsilon^{0123}=1$. Em 3 dimensões, definimos o tensor totalmente anti-simétrico de Levi-Civita $\epsilon^{i j k}=\epsilon^{0 i j k}$.

Abreviações:

- QED: Quantum electrodynamics (Eletrodinâmica quântica).

- QCD: Quantum chromodynamics (Cromodinâmica quântica).

- IR: Infrared (Infravermelho). 


\section{Capítulo 2}

\section{Introdução}

Em 2013, foi publicado um artigo [1] sobre eletrodinâmica não linear no regime estacionário (em campo de fundo nulo). Nele apresentamos uma generalização da Lei de Coulomb e da Lei de BiotSavart levando em conta o efeito de polarização do vácuo, aplicados a distribuições simples, como uma esfera homogeneamente carregada e uma esfera homogeneamente magnetizada. Essa dissertação é uma continuação natural desse artigo, onde discutimos e generalizamos os resultados obtidos, e aplicamos a corpos intensamente carregados, como estrelas de quarks, e corpos fortemente magnetizados, como pulsares, os tratando com modelos simplificados. No entanto, várias questões ficaram em aberto, como por exemplo o tratamento analítico de problemas que foram resolvidos de forma aproximada no artigo (onde foi usado o cálculo perturbativo até terceira ordem), o que possibilitaria, tendo o campo elétrico exato (de acordo com o modelo considerado) em mãos, o cálculo das integrais envolvidas na energia eletrostática. Dessa forma, nessa dissertação não só encontramos uma expressão mais realística para o campo eletrostático coulombiano, como também mostramos que a introdução de um mínimo de não linearidade já implica na remoção das divergências que surgem naturalmente da teoria de Maxwell.

É bem aceito atualmente que a teoria que melhor descreve o eletromagnetismo é a QED [2], em que os campos elétricos e magnéticos são vistos como operadores atuando no espaço de Fock. Através do formalismo da ação efetiva [3], podemos calcular correções quânticas ao campo eletromagnético. $\mathrm{Na}$ teoria quântica de campos, a ação efetiva é uma expressão modificada para a ação, que considera as correções da mecânica quântica, da seguinte maneira: Na teoria clássica, as equações de movimento podem ser derivadas a partir da ação pelo princípio de mínima ação. Este não é o caso da mecânica quântica, onde as amplitudes de todos os movimentos possíveis são somados numa integração funcional. No entanto, se a ação é substituída pela ação efetiva, as equações de movimento para os valores esperados do vácuo dos campos pode ser calculada a partir da exigência de que a ação efetiva seja estacionária. Além disso, a ação efetiva pode ser usada ao invés da ação, no cálculo das funções de correlação.

Graças ao princípio da correspondência, a compreensão precisa e objetiva de toda teoria quântica se manifesta diante da possibilidade de identificar a sua teoria clássica correspondente. Essa teoria, por sua vez, quando submetida a um processo formal e rigoroso de quantização, implica na correspondente teoria quântica, conforme desenvolvido, por exemplo, em [4].

A eletrodinâmica quântica é uma teoria não linear que inclui interação efetiva entre campos eletromagnéticos, devido a criação de pares virtuais de partículas carregadas, elétrons e pósitrons, que interagem com tais campos antes de se aniquilarem. A não linearidade se torna usualmente essencial quando os campos eletromagnéticos envolvidos no problema alcançam e excedem o valor crítico de Schwinger [5] na ordem de $m^{2} / e$, onde $m$ e e são a massa do elétron e a carga. Este valor 
é $B_{S c h}=4,4 \times 10^{13} G$ para o campo magnético e $E_{S c h}=1,3 \times 10^{16} \mathrm{~V} / \mathrm{cm}$ para o campo elétrico. Ou seja, em outras palavras, se os campos eletromagnéticos envolvidos no problema são fracos, podemos usar as equações de Maxwell para encontrá-los, dada uma fonte. Elas são lineares, e providenciam a validade do princípio da superposição, isto é, eles não interagem entre si, e eles podem ser linearmente combinados independentemente. Mas se os campos eletromagnéticos são suficientemente fortes, há uma amplitude de probabilidade importante de criação de um par elétron-pósitron pelo campo, digamos um fóton. Como consequência, haverá espalhamento de fótons que darão origem a campos não lineares, ou seja, um mecanismo que permite campos eletromagnéticos interagirem entre si.

No ano de 2012, foi publicado um artigo sobre o efeito magneto-elétrico [6], que leva em conta efeitos não lineares da produção de um campo magnético, devido uma certa distribuição de cargas eletrostáticas em repouso, em um referencial de Lorentz especial, imersa em um campo magnético externo de fundo constante, além da produção de um campo elétrico coulombiano anisotrópico (devido à existência de uma direção privilegiada, que é a do campo de fundo). Este efeito é genuinamente quântico, e tem origem no espalhamento de fótons com 3-vértices, isto é, o diagrama de um fóton separando-se em dois (e também dois fótons unindo-se em um). Entretanto, quando levamos em conta um campo externo de fundo nulo, o diagrama de fótons com 4-vértices (espalhamento fótonpor-fóton) é o mecanismo responsável pela não linearidade. Este efeito magneto-elétrico tem sido verificado em estudos recentes sobre estrelas de quarks fortemente magnetizadas [7].

Neste contexto, estendemos o formalismo da ação efetiva, progredimos e generalizamos o estudo do efeito magneto-elétrico, tratando agora como meio o vácuo, isto é, quando tomamos o limite de campo externo de fundo nulo. Conforme mencionado no primeiro parágrafo, objetivamos investigar os fenômenos provenientes da polarização do vácuo, e quais são as implicações das correções quânticas à eletrodinâmica não-linear.

Em [8], diversos modelos de eletrodinâmica não linear são levados em conta. Nesta obra, o principal foco de estudo é a eletrodinâmica não linear invariante de calibre $U(1)$, que inclui eletrodinâmica quântica. Além disto, há outros modelos bem conhecidos, como, por exemplo, o modelo de Born-Infeld [9], que propicia a eletrodinâmica não linear no vácuo, e teoria não-comutativa de calibre $U_{\star}(1)$, considerada, por exemplo, em $[10,11]$. Como nossa primeira hipótese, estamos considerando a aproximação infravermelho, isto é, que toma em conta o limite local do campo de fundo constante, e os campos variam lentamente. Quando efeitos não lineares são considerados, com esse campo externo constante de fundo, e aplicados a uma certa distribuição de cargas em repouso, em um referencial de Lorentz especial, suponhamos um campo magnético, o efeito magneto-elétrico surge e o meio pode ser tratado como anisotrópico, onde o campo coulombiano também apresenta anisotropia [6]. Este fenômeno é devido ao tensor de polarização de terceira ordem, cuja origem está no espalhamento de fótons com 3 vértices, isto é, não apenas espalhamento fóton-por-fóton, mas também o diagrama de um fóton separando-se em dois (também dois fótons unindo-se em um).

Entretanto, quando levamos em conta um campo nulo de fundo, o tensor de polarização de terceira ordem é zero, e o tensor de polarização de quarta ordem surge como a primeira correção não linear do campo. Neste caso, o diagrama de fótons com 4 vértices é o mecanismo responsável pela não linearidade. Além disso, a resposta linear (tensor de polarização de segunda ordem) é zero quando levamos em conta a ausência de campos externos de fundo.

As condições para que tais campos existam estão presentes em corpos celestes fortemente carregados ou magnetizados. Além da habitual referência a campos magnéticos suficientemente fortes que existiram no início do Universo [12], ou estão existindo [13, 14] em pulsares e magnetars, foi recentemente descoberto que os campos elétricos acima do valor crítico de Schwinger são esperados [15] em estrelas de quarks, e estes campos magnéticos intensos são também formados por um curto tempo quando partículas carregadas aceleradas (íons pesados) colidem com um parâmetro de impacto não 
nulo [16]. Nesta dissertação estimamos o efeito da não-linearidade na magnetosfera de pulsares e magnetars, avaliando a correção quântica que é atribuida ao campo estacionário, calculando modelos simplificados como uma esfera uniformemente magnetizada, bem como o campo elétrico em estrelas de quarks, tomando como modelo um monopolo elétrico uniformemente carregado.

A partir do formalismo da ação efetiva, que é não linear, introduzimos a noção da corrente não linearmente induzida, em termos do campo eletromagnético no caso mais geral, após a obtenção das equações de Euler-Lagrange. Com esta corrente em mãos, estudamos alguns casos estacionários, onde resolvemos as equações não lineares da eletrostática e magnetostática, cuja fonte é uma corrente não linearmente induzida e, além disto, estacionária, consideramos uma modificação invariante de calibre da eletrodinâmica clássica (quártica no campo) que possui uma regularização que torna a energia de uma carga puntiforme finita. Aqui consideramos teorias que incluem auto-interações, como o modelo de Euler-Heisenberg e de Born-Infeld. Este modelo admite soluções exatas para campos centralmente simétricos. Atendo-se ao caso eletrostático, i.e., o problema de Coulomb, pretendemos calcular de forma analítica a correção quântica à Lei de Coulomb, e então calcular a energia eletrostática contida em uma carga puntiforme, e verificar que a não linearidade sana o problema da catástrofe ultravioleta da teoria de Maxwell, evidenciando que há uma dependência dessa energia com a constante de acoplamento que a teoria dispõe, mesmo no regime onde a não linearidade é mínima (modelo quártico). Expandindo a solução para o campo em séries de potências, encontramos o campo não linear como uma perturbação, que produz uma correção até terceira ordem do campo eletromagnético. Aplicamos a correção ao campo devido a um monopolo e a um dipolo, tanto elétrico quanto magnético, e discutimos sobre o aspecto singular da teoria não linear. Como exemplo já existente na literatura [2], mostramos que o campo não linear $E$ produzido por uma carga $q$ a uma distância $r$ da origem é dado por:

$$
E=\mathcal{E}\left(1-\frac{2 \alpha}{45 \pi}\left(\frac{e \mathcal{E}}{m^{2}}\right)^{2}\right)
$$

onde $\mathcal{E}=q /\left(4 \pi r^{2}\right)$ é a intensidade do campo elétrico de Coulomb no regime linear [17], $\alpha \approx 1 / 137$ é a constante de estrutura fina, $e$ o módulo da carga elementar do elétron, e $m$ a massa de repouso do elétron.

Como uma aplicação, consideramos os casos onde as correções são consideráveis, como dipolos magnéticos que modelam pulsares [19], porque eles possuem uma magnetização intensa, quando comparado a outros corpos celestes no universo visível, como o caso do magnetar SGR 0418+5729 [20], que possui um campo magnético na sua superfície em torno de $1 / 4$ do valor crítico de Schwinger. Estima-se que efeitos não lineares são observáveis também em monopolos elétricos, que modelam estrelas de quarks $[25,15]$, cujos campos estão na ordem de grandeza de $10^{18}-10^{19} \mathrm{~V} / \mathrm{cm}$, e núcleos de átomos não tão pesados, suficientemente perto deles. Algumas estimativas numéricas serão consideradas neste mesmo capítulo para comparar os dados experimentais [26], para investigar a validade deste modelo, levando em conta a lagrangeana de Euler-Heisenberg, que é uma aproximação assintótica em eletrodinâmica quântica, para campos fracos. Se os campos envolvidos são maiores que o valor característico de Schwinger $\left(\mathrm{m}^{2} / e\right)=4,4 \cdot 10^{13}$ no sistema de unidades cgse [5], isto é, $1,3 \cdot 10^{16}$ $\mathrm{V} / \mathrm{cm}$ quando se mede um campo elétrico, e $4,4 \cdot 10^{13} \mathrm{G}$ quando se mede um campo magnético, o vácuo se torna instável, e é necessário levar em conta o efeito de Schwinger da criação espontânea de pares elétron-pósitron [27]. Estima-se que efeitos não lineares são observáveis também em monopolos elétricos, que modelam estrelas de quarks [25]. Para estrela de quarks do tipo strange, quando a matéria está no estado supercondutor [28], este limite pode ser atingido na sua superfície [29]. 


\section{Capítulo 3}

\section{Eletrodinâmica não linear em um campo de fundo constante}

Para campos externos de fundo constantes, há uma renormalização das intensidades do campo e de uma corrente que resulta em uma função de Green para o campo gerado por uma partícula imersa nesse campo eletromagnético prescrito. A corrente de vácuo associada a diagramas de espalhamento de fótons é descrita através da variação de uma ação, implicando em um comportamento não linear do campo.

Nós descrevemos a ação efetiva deste problema como a transformação de Legendre das funções de Green [3], que são, por sua vez, um funcional gerador de vértices redutíveis a uma partícula, que é fornecido por alguma teoria de QED. Podemos impor o princípio da mínima ação para encontrar as equações de Euler-Lagrange, e elas providenciam equações para o campo eletromagnético, cuja parte linear é equivalente às equações de Maxwell no limite de correntes fracas, de fato, mas em um meio equivalente produzido por um tensor de polarização, definido com a segunda derivada variacional da ação efetiva, tomada em um campo externo de fundo. A ação efetiva é dada por

$$
\Gamma=\int \mathfrak{L}(z) \mathrm{d}^{4} z
$$

onde $\mathfrak{L}$ é a lagrangeana efetiva, que depende dos invariantes relativísticos $\mathfrak{F}(x)=\frac{1}{4} F_{\mu \nu}(x) F^{\mu \nu}(x)$, $\mathfrak{G}(x)=\frac{1}{4} F_{\mu \nu}(x) \tilde{F}^{\mu \nu}(x)$, onde as intensidades dos campos são $F_{\mu \nu}(x)=\partial_{\mu} A_{\nu}(x)-\partial_{\nu} A_{\mu}(x), A_{\mu}(x)$ são os campos potenciais, e $\tilde{F}_{\mu \nu}(x)=\frac{1}{2} \epsilon_{\mu \nu \alpha \beta} F^{\alpha \beta}(x)$ é seu tensor dual. Podemos pensar sobre quaisquer lagrangeanas, mas não estamos considerando a dependência nas derivadas do campo. Conforme mencionado anteriormente, assumimos a hipótese dos campos que variam lentamente. Assim, podemos calcular o tensor de quarta ordem, e portanto a corrente não linearmente induzida, que é responsável pela correção do campo eletromagnético, tendo em mente que o tensor de terceira ordem é nulo na aproximação de ausência de campo externo de fundo.

Para encontrar e resolver as equações de Euler-Lagrange na qual $A^{\mu}(x)$ deve satisfazer, dada uma corrente $J_{\beta}(x)$, então precisamos aplicar o princípio variacional à ação total $S_{t o t}$, definida por

$$
\begin{aligned}
S_{\text {tot }} & =S_{\text {livre }}+S_{\text {int }}+\Gamma, \\
S_{\text {free }} & =-\int \mathfrak{F}(z) \mathrm{d}^{4} z, \\
S_{\text {int }} & =-\int J_{\beta}(z) A^{\beta}(z) \mathrm{d}^{4} z .
\end{aligned}
$$


Este princípio impõe mínima ação de $S_{\text {tot }}$, i.e., $\frac{\delta S_{\text {tot }}}{\delta A^{\beta}(x)}=0$, portanto $-\frac{\delta S_{\text {int }}}{\delta A^{\beta}(x)}=\frac{\delta S_{\text {livre }}}{\delta A^{\beta}(x)}+\frac{\delta \Gamma}{\delta A^{\beta}(x)}$, e obtemos as equações de Euler-Lagrange

$$
J_{\mu}(z)=\frac{\delta S_{\text {livre }}}{\delta A^{\mu}(x)}+\frac{\delta \Gamma}{\delta A^{\mu}(x)} .
$$

Subdividindo esta corrente em duas partes: $J_{\beta}(x)=j_{\beta}(x)+\mathcal{J}_{\beta}(x)$, onde $\mathcal{J}_{\beta}(x)=\left.\frac{\delta S}{\delta A^{\beta}(x)}\right|_{A=\mathcal{A}}$, tal que $S=S_{\text {livre }}+\Gamma$, e $\mathcal{A}^{\beta}(x)$ representa o campo externo, devemos encontrar soluções na forma $A^{\beta}(x)=\mathcal{A}^{\beta}(x)+a^{\beta}(x)$. Nossa primeira hipótese é sobre $a^{\beta}(x)$, que deve ser pequeno o suficiente para que possamos expandir $\frac{\delta \Gamma}{\delta A^{\beta}(x)}$ em potências. Assim, podemos expandir a ação não linear em séries de potencias de $a$, em torno do ponto $A=\mathcal{A}$, e então (3.3) fornece ${ }^{1}$ (veja os cálculos no Apêndice A1)

$$
\begin{aligned}
j_{\mu}(x) & =\left[\square \eta_{\mu \tau}-\partial_{\mu} \partial_{\tau}\right] a^{\tau}(x)+\int \mathrm{d}^{4} y \Pi_{\mu \tau}(x, y) a^{\tau}(y) \\
& +\frac{1}{2} \int \mathrm{d}^{4} y \mathrm{~d}^{4} u \Pi_{\mu \tau \sigma}(x, y, u) a^{\tau}(y) a^{\sigma}(u) \\
& +\frac{1}{6} \int \mathrm{d}^{4} y \mathrm{~d}^{4} u \mathrm{~d}^{4} v \Pi_{\mu \tau \sigma \rho}(x, y, u, v) a^{\tau}(y) a^{\sigma}(u) a^{\rho}(v)+\ldots
\end{aligned}
$$

onde $j_{\mu}(x)^{2}$ é uma fonte pequena do campo, e

$$
\begin{gathered}
\Pi_{\mu \tau}\left(x, x^{\prime}\right)=\left.\frac{\delta^{2} \Gamma}{\delta A^{\mu}(x) \delta A^{\tau}\left(x^{\prime}\right)}\right|_{A=\mathcal{A}}, \\
\Pi_{\mu \tau \sigma}\left(x, x^{\prime}, x^{\prime \prime}\right)=\left.\frac{\delta^{3} \Gamma}{\delta A^{\mu}(x) \delta A^{\tau}\left(x^{\prime}\right) \delta A^{\sigma}\left(x^{\prime \prime}\right)}\right|_{A=\mathcal{A}}, \\
\Pi_{\mu \tau \sigma \rho}\left(x, x^{\prime}, x^{\prime \prime}, x^{\prime \prime \prime}\right)=\left.\frac{\delta^{4} \Gamma}{\delta A^{\mu}(x) \delta A^{\tau}\left(x^{\prime}\right) \delta A^{\sigma}\left(x^{\prime \prime}\right) \delta A^{\rho}\left(x^{\prime \prime \prime}\right)}\right|_{A=\mathcal{A}}, \\
\text { etc. }
\end{gathered}
$$

Podemos calcular (3.5) e (3.6) aplicando repetidamente a relação (veja os cálculos no Apêndice A2)

$$
\frac{\delta \Gamma}{\delta A^{\mu}(x)}=\int\left[\frac{\partial \mathfrak{L}(\mathfrak{F}(z), \mathfrak{G}(z))}{\partial \mathfrak{F}(z)} F_{\alpha \mu}(z)+\frac{\partial \mathfrak{L}(\mathfrak{F}(z), \mathfrak{G}(z))}{\partial \mathfrak{G}(z)} \tilde{F}_{\alpha \mu}(z)\right] \frac{\partial}{\partial z_{\alpha}} \delta^{4}(x-z) \mathrm{d}^{4} z .
$$

Dizemos que o tensor de polarização de segunda ordem $\Pi_{\mu \tau}$ é a resposta linear à corrente $j_{\mu}$, pois é um operador que atua em $a$, enquanto que os tensores de polarização de ordens superiores $\Pi_{\mu \tau \sigma}, \Pi_{\mu \tau \sigma \rho}$, etc. são a resposta não linear a essa corrente, onde cada um desses tensores atuam em

\footnotetext{
${ }^{1} \mathrm{O}$ operador $\nabla$, o Laplaciano $\triangle$, e o d'Alembertiano $\square$ são definidos por:

$$
\begin{aligned}
& \nabla=\mathbf{e}_{i} \nabla_{i}=\mathbf{e}_{i} \partial_{i}=\left(\partial_{i}\right), \\
& \triangle=\nabla^{2}=\partial_{i}^{2}, \square=\partial_{\mu} \partial^{\mu}=\partial_{0}^{2}-\triangle .
\end{aligned}
$$

${ }^{2}$ A quadricorrente é definida por $j^{\mu}(x)=\left(j^{0}(x), \mathbf{j}(x)\right)$, onde $j^{0}(x)$ denota a densidade de cargas, e $\mathbf{j}(x)$, a densidade de corrente. Analogamente, $a^{\mu}(x)=\left(a^{0}(x), \mathbf{a}(x)\right)$
} 
uma potência de em a que é dada cada um pela sua respectiva ordem decrescida de uma unidade, conforme exposto em (3.4), por exemplo, $\Pi_{\mu \tau \sigma}$ atua em um termo quadrático de $a$, e $\Pi_{\mu \tau \sigma \rho}$ atua em um termo cúbico de $a$, e assim por diante.

Daqui em diante, trataremos apenas dos campos externos $\mathcal{F}_{\alpha \beta}=\partial_{\alpha} \mathcal{A}_{\beta}^{\text {ext }}-\partial_{\beta} \mathcal{A}_{\alpha}^{\text {ext }}$, que são constantes no espaço-tempo. Além disto, para QED e outras teorias de interesse, se a lagrangeana efetiva $\mathfrak{L}(z)$ depende da coordenada $z_{\mu}$, é apenas devido ao campo tensorial $F_{\alpha \beta}(z)$ e suas derivadas espaçotemporais, e não explicitamente. Esta hipótese implica que o campo constante satisfaz exatamente a equação do campo na ausência de fontes $\left.\frac{\delta S}{\delta A^{\beta}(x)}\right|_{A=\mathcal{A}}=0$, porque estamos assumindo que o campo externo está sujeito à equação de campos sem fontes. A razão é que a derivada variacional atuando nele é

$$
\left.\frac{\delta S}{\delta A_{\beta}(x)}\right|_{A=\mathcal{A}_{\text {ext }}}=\left.2 \sum_{n} \int \frac{\delta S}{\delta F_{\alpha \beta}^{(n)}(z)}\right|_{F=\mathcal{F}} \frac{\partial}{\partial z^{\alpha}} \delta^{4(n)}(x-z) \mathrm{d}^{4} z .
$$

Entretanto, quando tomamos o campo constante $F_{\mu \nu}(z)=\mathcal{F}_{\mu \nu}$, a derivada variacional $\frac{\delta S}{\delta F_{\alpha \beta}^{(n)}(z)}$ não depende de $z$, e por integração por partes, esta integral é zero.

Por esta razão, (3.4) é a equação do campo para pequenos campos $a^{\beta}(x)=A^{\beta}(x)-\mathcal{A}^{\beta}(x)$ sob o campo externo de uma intensidade do campo $\mathcal{A}^{\beta}(x)$ constante, causado por uma corrente $j_{\rho}(x)$.

A invariância sobre transformação de calibre da expansão de $\Gamma$, em potências do campo $a^{\nu}(x)$, é garantida pela propriedade de transversalidade dos tensores de polarização de quaisquer ordens a $\Pi_{\mu \tau \ldots \sigma}\left(x, x^{\prime}, \ldots x^{\prime \prime}\right)$, que satisfazem as relações de continuidade com respeito a cada argumentos $\mathrm{e}$ índices

$$
\frac{\partial}{\partial x_{\tau}^{\prime}} \Pi_{\mu \ldots \tau \ldots \sigma}\left(x, \ldots x^{\prime}, \ldots x^{\prime \prime}\right)=0
$$

necessárias para providenciar a invariância de cada termo na expansão de $\Gamma$ em potências do campo $a^{\nu}(x)$ sobre sua transformação de calibre. Isto implica no fato de a invariância translacional permanecer válida, desde que $\Gamma$ dependa das intensidades do campo e suas derivadas espaço-temporais somente, e estamos interessados em campos externos que são independentes das coordenadas espaçotemporais. Como uma consequência, tensores de polarização de quaisquer ordens dependem da diferença entre as coordenadas.

Com a definição do propagador de fótons $D_{\mu \nu}\left(x, x^{\prime}\right)$

$$
D_{\mu \nu}^{-1}\left(x-x^{\prime}\right)=\left[\eta_{\mu \nu} \square-\partial_{\mu} \partial_{\nu}\right] \delta^{(4)}\left(x^{\prime}-x\right)+\Pi_{\mu \nu}\left(x-x^{\prime}\right),
$$

as equações para os campos não lineares (3.4) tomam forma do conjunto de equações integrais

$$
a^{\lambda}(x)=\int d^{4} y D^{\lambda \rho}(x-y) j_{\rho}(y)+\int d^{4} y D^{\lambda \rho}(x-y) j_{\rho}^{\mathrm{nl}}(y),
$$

tal que

$$
\begin{aligned}
& j_{\mu}^{\mathrm{nl}}(x)=-\frac{1}{2} \int \mathrm{d}^{4} y \mathrm{~d}^{4} u \Pi_{\mu \tau \sigma}(x, y, u) a^{\tau}(y) a^{\sigma}(u) \\
& -\frac{1}{6} \int \mathrm{d}^{4} y \mathrm{~d}^{4} u \mathrm{~d}^{4} v \Pi_{\mu \tau \sigma \rho}(x, y, u, v) a^{\tau}(y) a^{\sigma}(u) a^{\rho}(v) \\
& + \text { termos de ordens superiores, }
\end{aligned}
$$

onde definimos a corrente não linearmente induzida $j_{\mu}^{\mathrm{nl}}(x)$. 


\section{Capítulo 4}

\section{Efeito magneto-elétrico}

Neste capítulo descrevemos o problema de uma carga em repouso em um certo referencial, sob um campo magnético de fundo uniforme e constante, dentro da aproximação infravermelho, descrita

a seguir. A correção não linear ao campo é puramente magnética. É o que denominamos efeito magneto-elétrico [30].

\subsection{Aproximação infravermelho}

A partir de agora, devemos nos restringir somente a campos $a^{\lambda}(x)$ que variam lentamente e, correspondentemente, à consideração de fontes $j_{\rho}(x)$ que dão origem a tais campos por intermédio das equações (3.11), (3.12). Para este fim, tomamos a ação efetiva no seu limite local, onde as dependências nas derivadas do campo são desconsideradas nesse funcional.

Daqui em diante, estaremos restritos em um caso especial, quando o campo externo de fundo é constante e puramente magnético em uma certa classe de referenciais, chamados aqui de especiais. Como em outros referenciais de Lorentz o campo elétrico está também presente, nos referimos a esse caso como caso magnético. As condições a serem impostas aos campos invariantes para especializar o caso magnético são $\mathfrak{F}>0, \mathfrak{G}=0$. Uma vez que o invariante $\mathfrak{G}$ é pseudoescalar, a lagrangeana de uma teoria P-invariante, incluindo QED, pode conte-la apenas em uma potência par. Assim, todas derivadas ímpares de $\mathfrak{L}(\mathfrak{F}, \mathfrak{G})$ em relação a este invariante desaparecem depois da adoção do campo no caso magnético, no limite de campo externo constante:

$$
\left.\frac{\partial \mathfrak{L}(\mathfrak{F}, \mathfrak{G})}{\partial \mathfrak{G}}\right|_{F=\mathcal{F}, \mathfrak{G}=0}=\left.\frac{\partial^{2} \mathfrak{L}(\mathfrak{F}, \mathfrak{G})}{\partial \mathfrak{G} \partial \mathfrak{F}}\right|_{F=\mathcal{F}, \mathfrak{G}=0}=\left.\frac{\partial^{3} \mathfrak{L}(\mathfrak{F}, \mathfrak{G})}{\partial \mathfrak{G} \partial \mathfrak{F}^{2}}\right|_{F=\mathcal{F}, \mathfrak{G}=0}=\left.\frac{\partial^{3} \mathfrak{L}(\mathfrak{F}, \mathfrak{G})}{\partial \mathfrak{G}^{3}}\right|_{F=\mathcal{F}, \mathfrak{G}=0}=0 .
$$

Logo, levando (4.1) em conta e usando as notações

$$
\begin{aligned}
& \mathfrak{L}_{\mathfrak{F}}=\left.\frac{\mathrm{d} \mathfrak{L}(\mathfrak{F}, 0))}{\mathrm{d} \mathfrak{F}}\right|_{F=\mathcal{F}}, \quad \mathfrak{L}_{\mathfrak{F} \mathfrak{F}}=\left.\frac{\mathrm{d}^{2} \mathfrak{L}(\mathfrak{F}, 0)}{\mathrm{d} \mathfrak{F}^{2}}\right|_{F=\mathcal{F}}, \quad \mathfrak{L}_{\mathfrak{G} \mathfrak{G}}=\left.\frac{\partial^{2} \mathfrak{L}(\mathfrak{F}, \mathfrak{G})}{\partial \mathfrak{G}^{2}}\right|_{F=\mathcal{F}, \mathfrak{G}=0}, \\
& \mathfrak{L}_{\mathfrak{F} \mathfrak{F} \mathfrak{F}}=\left.\frac{\mathrm{d}^{3} \mathfrak{L}(\mathfrak{F}, 0)}{\mathrm{d} \mathfrak{F}^{3}}\right|_{F=\mathcal{F}}, \quad \mathfrak{L}_{\mathfrak{F} \mathfrak{G} \mathfrak{G}}=\left.\frac{\mathrm{d}}{\mathrm{d} \mathfrak{F}} \frac{\partial^{2} \mathfrak{L}(\mathfrak{F}, \mathfrak{G})}{\partial \mathfrak{G}^{2}}\right|_{F=\mathcal{F}, \mathfrak{G}=0},
\end{aligned}
$$

todos com a substituição $F_{\mu \nu}=\mathcal{F}_{\mu \nu}$ (assim, agora, $\mathfrak{F}=\frac{1}{4} \mathcal{F}_{\rho \sigma} \mathcal{F}^{\rho \sigma}$ e $\mathfrak{G}=\frac{1}{4} \mathcal{F}^{\rho \sigma} \tilde{\mathcal{F}}_{\rho \sigma}=0$ ), nós obtemos o tensor de polarização de segunda ordem 


$$
\begin{aligned}
& \Pi_{\mu \tau}^{\mathrm{IR}}(x-y)=\mathfrak{L}_{\mathfrak{F}}\left(\frac{\partial^{2}}{\partial x^{\tau} \partial x^{\mu}}-\eta_{\mu \tau} \square\right) \delta^{4}(x-y) \\
& -\left\{\mathfrak{L}_{\mathfrak{F F}} \mathcal{F}_{\alpha \mu} \mathcal{F}_{\beta \tau}+\mathfrak{L}_{\mathfrak{G G} \mathfrak{\mathcal { F }}} \tilde{\mathcal{F}}_{\alpha \mu} \tilde{\mathcal{F}}_{\beta \tau}\right\} \frac{\partial}{\partial x_{\alpha}} \frac{\partial}{\partial x_{\beta}} \delta^{4}(x-y),
\end{aligned}
$$

e o tensor de polarização de terceira ordem no limite infravermelho

$$
\begin{aligned}
& \Pi_{\mu \tau \sigma}^{\mathrm{IR}}(x-u, y-u)= \\
& =-\mathcal{O}_{\mu \tau \sigma \alpha \beta \gamma} \frac{\partial}{\partial x_{\alpha}}\left(\left(\frac{\partial}{\partial x_{\beta}} \delta^{4}(y-x)\right)\left(\frac{\partial}{\partial x_{\gamma}} \delta^{4}(x-u)\right)\right),
\end{aligned}
$$

onde

$$
\begin{aligned}
& \mathcal{O}_{\mu \tau \sigma \alpha \beta \gamma}=\mathfrak{L}_{\mathfrak{G G}}\left(\widetilde{\mathcal{F}}_{\gamma \sigma} \epsilon_{\alpha \mu \beta \tau}+\widetilde{\mathcal{F}}_{\alpha \mu} \epsilon_{\beta \tau \gamma \sigma}+\widetilde{\mathcal{F}}_{\beta \tau} \epsilon_{\alpha \mu \gamma \sigma}\right) \\
& +\mathfrak{L}_{\mathfrak{F F} \mathfrak{F}}\left[\left(\eta_{\mu \tau} \eta_{\alpha \beta}-\eta_{\mu \beta} \eta_{\alpha \tau}\right) \mathcal{F}_{\gamma \sigma}+\mathcal{F}_{\alpha \mu}\left(\eta_{\tau \sigma} \eta_{\gamma \beta}-\eta_{\beta \sigma} \eta_{\gamma \tau}\right)+\mathcal{F}_{\beta \tau}\left(\eta_{\mu \sigma} \eta_{\gamma \alpha}-\eta_{\alpha \sigma} \eta_{\gamma \mu}\right)\right] \\
& +\mathfrak{L}_{\mathfrak{F} \mathfrak{G G}}\left(\left[\mathcal{F}_{\alpha \mu} \widetilde{\mathcal{F}}_{\beta \tau} \widetilde{\mathcal{F}}_{\gamma \sigma}+\widetilde{\mathcal{F}}_{\alpha \mu} \mathcal{F}_{\beta \tau} \widetilde{\mathcal{F}}_{\gamma \sigma}+\widetilde{\mathcal{F}}_{\alpha \mu} \widetilde{\mathcal{F}}_{\beta \tau} \mathcal{F}_{\gamma \sigma}\right]\right)+\mathfrak{L}_{\mathfrak{F F F}} \mathcal{F}_{\alpha \mu} \mathcal{F}_{\beta \tau} \mathcal{F}_{\gamma \sigma} .
\end{aligned}
$$

As duas condições de transversalidade (3.9) para (4.2) implicam na antissimetria sob cada permutação de índices $\tau \Longleftrightarrow \beta$ and $\sigma \Longleftrightarrow \gamma$ no tensor (4.4). Graças a essas duas antissimetrias, usando (4.3), (4.4) em (3.12), obtemos a corrente não linearmente induzida

$$
j_{\mu}^{\mathrm{nl}}(x)=-\frac{1}{8} \mathcal{O}_{\mu \tau \sigma \alpha \beta \gamma} \frac{\partial}{\partial x_{\alpha}}\left(f^{\beta \tau}(x) f^{\gamma \sigma}(x)\right),
$$

onde definimos $f^{\beta \tau}(x)$ como o dobro da parte antissimétrica de $\frac{\partial a^{\tau}(x)}{\partial x_{\beta}}$, i.e., $f^{\beta \tau}(x)=\frac{\partial a^{\tau}(x)}{\partial x_{\beta}}-\frac{\partial a^{\beta}(x)}{\partial x_{\tau}}$, notando que a parte simétrica de $\frac{\partial a^{\tau}(x)}{\partial x_{\beta}}$ se torna zero após a contração com o tensor $\mathcal{O}_{\mu \tau \sigma \alpha \beta \gamma}$. Portanto, a corrente não linearmente induzida é invariante de calibre: Ela depende somente das intensidades do campo, e é conservada, i.e., $\partial^{\mu} j_{\mu}^{\mathrm{nl}}(x)=0$, devida à antissmetria pela troca $\mu \Longleftrightarrow \alpha$, em (4.5).

Aproximamos o conjunto de equações não lineares (3.11), (3.12) procurando sua solução por perturbação, i.e., em uma série de potências do campo $a^{\lambda}(x)$. Na primeira iteração, substituimos a aproximação linear à solução de (3.11)

$$
a_{\nu}^{\operatorname{lin}}(x)=\int d x^{\prime} D_{\nu \rho}\left(x-x^{\prime}\right) j^{\rho}\left(x^{\prime}\right)
$$

para $a(x)$ em (3.12). Em outras palavras, devemos usar o campo eletromagnético $f_{\beta \tau}=\frac{\partial a_{\tau}^{\operatorname{lin}}(x)}{\partial x^{\beta}}-$ $\frac{\partial a_{\beta}^{\operatorname{lin}}(x)}{\partial x^{\tau}}$ linearmente produzido pela fonte $j_{\mu}(x)$ na expressão da corrente não linearmente induzida $(4.5)$.

\subsection{Campo magnético de uma carga estática em um campo magnético externo de fundo}

Nesta seção estudamos o efeito não linear da produção de um campo magnético por uma carga estática em repouso em um campo magnético constante e uniforme em um referencial especial. O 
efeito linear do campo magnético externo no campo eletrostático da carga pode ser encontrado (além da aproximação infravermelho) em [32, 33, 34].

Neste referencial, o campo magnético externo, definido por $B_{i}=(1 / 2) \epsilon_{i j k} \mathcal{F}^{j k}$, enquanto, por sua vez, o campo elétrico é nulo $E_{i}=\mathcal{F}_{0 i}=0$.

Considere agora uma carga puntiforme dada, neste referencial, pela quadricorrente $j_{\mu}(x)=$ $j_{\mu}(\mathbf{r}) \delta_{\mu 0}$. Na aproximação linear (4.6), naturalmente, somente um campo eletrostático é gerado neste referencial. Assim, as componentes nas quais $\alpha=\beta=\gamma=0, \tau, \sigma \neq 0$ não contribuem para (4.5), portanto precisamos apenas das componentes

$$
\mathcal{O}_{i 00 j m n}=\mathcal{F}_{j i}\left[-\delta_{m n} \mathfrak{L}_{\mathfrak{F} \mathfrak{F}}+\widetilde{\mathcal{F}}_{n 0} \widetilde{\mathcal{F}}_{m 0} \mathfrak{L}_{\mathfrak{F} \mathfrak{G} \mathfrak{G}}\right]-\left[\widetilde{\mathcal{F}}_{m 0} \epsilon_{0 j i n}+\widetilde{\mathcal{F}}_{n 0} \epsilon_{0 j i m}\right] \mathfrak{L}_{\mathfrak{G G}},
$$

enquanto $\mathcal{O}_{000 j m n}=0$ de acordo com (4.4). $\operatorname{Logo}, j_{0}^{\text {nl }}(\mathbf{r})=0$, i.e., não há correção não linear (quadrática) à carga estática nesta aproximação: a corrente (4.5) é puramente espacial:

$$
\begin{aligned}
& j_{i}^{\mathrm{nl}}(\mathbf{r})=-\frac{1}{4} \mathcal{O}_{i 00 j m n} \frac{\partial}{\partial x_{j}}\left(f_{m 0} f_{n 0}\right)= \\
& =\frac{1}{4}\left[\boldsymbol{\nabla} \times\left[\left(\mathfrak{L}_{\mathfrak{F} \mathfrak{F}} \mathcal{E}^{2}(\mathbf{r})-\mathfrak{L}_{\mathfrak{F} \mathfrak{G} \mathfrak{G}}(\mathbf{B} \cdot \mathcal{E}(\mathbf{r}))^{2}\right) \mathbf{B}\right]\right]_{i}-\frac{1}{2} \mathfrak{L}_{\mathfrak{G G} \mathfrak{G}}[\boldsymbol{\nabla} \times[(\mathbf{B} \cdot \mathcal{E}(\mathbf{r}))] \mathcal{E}(\mathbf{r})]_{i},
\end{aligned}
$$

onde $\mathcal{E}_{n}(\mathbf{r})=f_{n 0}^{\operatorname{lin}}(\mathbf{r})=\frac{\partial a_{0}^{\operatorname{lin}}(\mathbf{r})}{\partial x_{n}}$ é o campo eletrostático, linearmente produzido em (4.6). O campo magnético $\boldsymbol{h}(\mathbf{r})$ gerado por esta corrente, de acordo com a equação de Maxwell $\boldsymbol{\nabla} \times \boldsymbol{h}(\mathbf{r})=\mathbf{j}^{\mathrm{nl}}(\mathbf{r})$ é

$$
h_{i}(\mathbf{r})=\mathfrak{h}_{i}(\mathbf{r})+\nabla_{i} \Omega(\mathbf{r}),
$$

onde

$$
\mathfrak{h}_{i}(\mathbf{r})=\frac{B_{i}}{4}\left[\mathfrak{L}_{\mathfrak{F} \mathfrak{F}}(\mathcal{E}(\mathbf{r}))^{2}-\mathfrak{L}_{\mathfrak{F} \mathfrak{G} \mathfrak{G}}(\boldsymbol{B} \cdot \mathcal{E}(\mathbf{r}))^{2}\right]-\frac{\mathcal{E}_{i}(\mathbf{r})}{2} \mathfrak{L}_{\mathfrak{G} \mathfrak{G}}(\boldsymbol{B} \cdot \mathcal{E}(\mathbf{r})),
$$

pois $\boldsymbol{\nabla} \times \boldsymbol{\nabla} \Omega(\mathbf{r}) \equiv 0$, e a função escalar $\Omega(\mathbf{r})$ está sujeita à equação de Poisson

$$
\nabla^{2} \Omega(\mathbf{r})=-\nabla_{j} \mathfrak{h}_{j}(\mathbf{r})
$$

para fazer o campo magnético $\boldsymbol{h}(\mathbf{r})$ obedecer a outra equação de Maxwell $\boldsymbol{\nabla} \cdot \boldsymbol{h}(\mathbf{r})=0$. Assim, o campo magnético é a parte transversal de (4.10) (veja as condições de existência e unicidade de $\boldsymbol{h}(\mathbf{r})$ nos apêndices $\mathrm{C}$ e D):

$$
h_{i}(\mathbf{r})=\left(\delta_{i j}-\frac{\nabla_{i} \nabla_{j}}{\nabla^{2}}\right) \mathfrak{h}_{j}(\mathbf{r})=\mathfrak{h}_{i}(\mathbf{r})+\frac{\nabla_{i} \nabla_{j}}{4 \pi} \int \frac{\mathfrak{h}_{j}\left(\mathbf{r}^{\prime}\right)}{\left|\mathbf{r}-\mathbf{r}^{\prime}\right|} d \mathbf{r}^{\prime}
$$

Isto coincidiria com o campo magnético $\mathbf{b}(\mathbf{r})=\boldsymbol{\nabla} \times \mathbf{a}^{\mathrm{nl}}(\mathbf{r})$ se a magnetização linear do vácuo puder ser desprezada, i.e., se a correção não linear em (3.11)

$$
a_{\mathrm{nl}}^{\lambda}(x)=\int d y D_{\rho}^{\lambda}(x-y) j_{\rho}^{\mathrm{nl}}(y)
$$

poder ser tomada sem a contribuição da função de resposta linear (4.3) $\Pi_{\mu \nu}\left(x-x^{\prime}\right)$ no propagador do fóton (3.10). Levando em conta esta contribuição, resultam-se integrais mais complicadas. A situação permanece simples, entretanto, quando podemos desconsiderar a anisotropia da resposta linear magnética. O tensor de permeabilidade magnética, inerente ao tensor de polarização de segunda ordem (4.2) é, no referencial especial [35], 


$$
\mu_{i j}^{-1}=\left(1-\mathfrak{L}_{\mathfrak{F}}\right) \delta_{i j}-\mathfrak{L}_{\mathfrak{F F}} B_{i} B_{j}
$$

cujos dois autovalores ${ }^{1} \mu_{\perp}^{-1}=1-\mathfrak{L}_{\mathfrak{F}}$, e $\mu_{\|}^{-1}=1-\mathfrak{L}_{\mathfrak{F}}-\mathfrak{L}_{\mathfrak{F F}} B^{2}$ são responsáveis pelas magnetizações linearmente causadas por certa corrente constante fluindo ao longo do campo magnético externo, e perpendicular a ele, respectivamente (veja Apêndice em [36]). Em QED, os valores $\mathfrak{L}_{\mathfrak{F}}$ e $\mathfrak{L}_{\mathfrak{F} F} B^{2}$ são da ordem da constante de estrutura fina $\alpha \approx 1 / 137$, mas depende do campo $B$. Quando $B$ é muito intenso, $B \gg m^{2} / e$, onde $m$ e $e$ são a massa e carga do elétron, respectivamente, essas quantidades, como encontradas devido à lagrangeana efetiva de Euler-Heisenberg de um laço, se comporta como

$$
\mathfrak{L}_{\mathfrak{F}} \approx \frac{\alpha}{3 \pi} \ln \frac{e B}{m^{2}}, \quad \mathfrak{L}_{\mathfrak{F F}} B^{2} \approx \frac{\alpha}{3 \pi} .
$$

Logo, quando $\frac{e B}{m^{2}} \gg 2,7$, a contribuição de $\mathfrak{L}_{\mathfrak{F F}} B^{2}$ pode ser desprezada se comparada a $\mathfrak{L}_{\mathfrak{F}}$, e a magnetização linear se torna isotrópica, $\mu_{\perp}^{-1}=\mu_{\|}^{-1}$. Logo, neste limite, finalmente temos o campo magnético

$$
\mathbf{b}(\mathbf{r})=\left(1-\mathfrak{L}_{\mathfrak{F}}\right)^{-1} \mathbf{h}(\mathbf{r})
$$

\subsection{Lei de Coulomb anisotrópica}

O campo elétrico $\mathcal{E}(\mathbf{r})=-\nabla a_{0}^{\operatorname{lin}}(\mathbf{r})$ a ser substituido em (4.8) e (4.10) é aquele que é linearmente produzido via (4.6) por uma distribuição de carga estática dentro da mesma aproximação infravermelho. Para determiná-lo, note que em (4.6) somente o componente $D_{00}$ do propagador participa, que, na representação de Fourier é $D_{00}=\left(\boldsymbol{k}^{2}-\varkappa_{2}\right)^{-1}$, com $\varkappa_{2}$ sendo um (dentre três) autovalor do tensor de polarização (3.5) tomado no limite estático $k_{0}=0$ no referencial especial. Uma vez que o tensor de polarização é considerado no seu limite infravermelho (4.2), esta quantidade é dada por $[36,37] \varkappa_{2}=\boldsymbol{k}^{2} \mathfrak{L}_{\mathfrak{F}}-k_{\|}^{2} 2 \mathfrak{F} \mathfrak{L}_{\mathfrak{G G} \mathfrak{G}}$. Aqui, $\boldsymbol{k}^{2}=\boldsymbol{k}_{\perp}^{2}+k_{\|}^{2}$, e $\boldsymbol{k}_{\perp}, k_{\|}$são os componentes do momento de um pequeno campo eletromagnético perpendicular e ao longo de $\boldsymbol{B}$, respectivamente. Agora, o cálculo de (4.6) para a carga puntiforme $j_{0}(\mathbf{r})=q \delta^{3}(\mathbf{r})$ resulta, com o uso deste propagador, na lei de Coulomb anisotrópica

$$
a_{0}^{\operatorname{lin}}(\mathbf{r})=\frac{q}{4 \pi} \frac{1}{\sqrt{\varepsilon_{\perp}} \sqrt{\varepsilon_{\perp} r_{\|}^{2}+\varepsilon_{\|} r_{\perp}^{2}}},
$$

onde $\varepsilon_{\perp}=1-\mathfrak{L}_{\mathfrak{F}}$ e $\varepsilon_{\|}=1-\mathfrak{L}_{\mathfrak{F}}+2 \mathfrak{F} \mathfrak{L}_{\mathfrak{G} \mathfrak{G}}$ são autovalores do tensor dielétrico [35] $\varepsilon_{i j}=\left(1-\mathfrak{L}_{\mathfrak{F}}\right) \delta_{i j}+$ $\mathfrak{L}_{\mathfrak{G G}} B_{i} B_{j}$, responsável pelas polarizações, linearmente causadas por planos carregados uniformemente paralelos e ortogonais a $\mathbf{B}$, respectivamente, e $r_{\perp}$ e $r_{\|}$são as componentes da coordenada perpendicular e ao longo de B. Para campos magnéticos intensos, obtemos a linearidade evoluindo assintóticamente para a lagrangeana de Euler-Heisenberg $2 \mathfrak{F} \mathfrak{L}_{\mathfrak{G} \mathfrak{G}} \approx \frac{\alpha}{3 \pi} \frac{e B}{m^{2}}$. Isto significa que se $\frac{e B}{m^{2}}>\frac{3 \pi}{\alpha}$ a componente dielétrica $\varepsilon_{\|}$domina sobre $\varepsilon_{\perp}$, i.e., a eletrização se torna altamente anisotrópica, em contraposição à magnetização acima. Nesta região assintótica, (4.15) se torna (se desconsiderarmos a polarização em $\varepsilon_{\perp}$ definindo $\varepsilon_{\perp}=1$ ) o comportamento a longas distâncias do potencial de uma carga puntiforme em um campo magnético intenso calculado na aproximação linear em [32, 33] além da aproximação infravermelho do tensor de polarização. Note que (4.15), bem como seu limite de campo forte, é válido somente longe o suficiente da carga. Neste domínio, no entanto, é também ajusta quaisquer distribuições de cargas, com carga total $q$, distribuida sobre uma região finita.

\footnotetext{
${ }^{1} \mathrm{O}$ campo magnético constante de fundo produz um meio uniaxial em qualquer referencial especial [35].
} 


\subsection{Diagrama de três fótons}

Neste capítulo, encontramos uma expressão para o campo magnético $\mathbf{h}(\mathbf{r})$ produzido por uma carga estática $q$ alocada em um campo magnético externo de fundo B, nas eqs. (4.10), (4.11). Mostramos que em QED este efeito magneto-elétrico ocorre já na aproximação mais simples, onde a lagrangeana efetiva $\mathfrak{L}$ é tomada no seu limite infravermelho, e somente a segunda potência da carga $q$ e/ou seu campo elétrico $\mathcal{E}(\mathbf{r})$ são mantidos. Como para o campo magnético de fundo $\mathbf{B}$, para notar o efeito basta tomar em conta a aproximação linear, $\sim B$, embora o campo magnético $B$ de intensidade arbitrária está incluído nestes resultados, também. As fórmulas finais dependem das primeiras três derivadas da ação efetiva $\mathfrak{L}$ com respeito aos invariantes do campo externo, que cumprem o fato de que, minimalmente, diagramas de três vértices de fótons são responsáveis pelo efeito nesta dada aproximação. 


\section{Capítulo 5}

\section{Problemas estacionários em um campo de fundo nulo}

Neste capítulo, tomamos o campo externo de fundo $\mathcal{F}_{\alpha \beta}$ como sendo zero em todo espaço, e escolhemos referenciais especiais onde podemos considerar problemas estacionários. Logo, as derivadas variacionais em $(3.5)$ e (3.6) podem ser calculadas em termos das derivadas de $\mathfrak{L}(\mathfrak{F}, \mathfrak{G})$ com respeito aos campos invariantes reduzidos a campo externos nulos. Pretendemos exibir, em primeira aproximação não linear, a corrente não linearmente induzida desse problema, tendo em mente que estamos sujeitos à aproximação infra-vermelho.

\subsection{Resposta linear e tensor de polarização de terceira or- dem}

De agora em diante, encontraremos uma equação para a corrente (3.4) em termos das intensidades de campo eletromagnéticos e das derivadas da lagrangeana $\mathfrak{L}(\mathfrak{F}(z), \mathfrak{G}(z))$, considerando que o campo externo de fundo é nulo. Definindo $\mathfrak{L}_{\mathfrak{F}}=\left.\frac{\partial \mathfrak{L}(\mathfrak{F}(z), \mathfrak{G}(z))}{\partial \mathfrak{F}}\right|_{\mathfrak{F}=0, \mathfrak{G}=0}, \mathfrak{L}_{\mathfrak{G}}=\left.\frac{\partial \mathfrak{L}(\mathfrak{F}(z), \mathfrak{G}(z))}{\partial \mathfrak{G}}\right|_{\mathfrak{F}=0, \mathfrak{G}=0}, \mathfrak{L}_{\mathfrak{F} \mathfrak{F}}=$ $\left.\frac{\partial^{2} \mathfrak{L}(\mathfrak{F}(z), \mathfrak{G}(z))}{\partial \mathfrak{F}^{2}}\right|_{\mathfrak{F}=0, \mathfrak{G}=0}, \quad \mathfrak{L}_{\mathfrak{F} \mathfrak{G}}=\left.\frac{\partial^{2} \mathfrak{L}(\mathfrak{F}(z), \mathfrak{G}(z))}{\partial \mathfrak{F} \partial \mathfrak{G}}\right|_{\mathfrak{F}=0, \mathfrak{G}=0}$ e $\mathfrak{L}_{\mathfrak{G} \mathfrak{G}}=\left.\frac{\partial^{2} \mathfrak{L}(\mathfrak{F}(z), \mathfrak{G}(z))}{\partial \mathfrak{G}^{2}}\right|_{\mathfrak{F}=0, \mathfrak{G}=0}$. Por $(3.5)$ e $(3.6)$, podemos calcular os seguintes tensores no vácuo polarizado (tomados em $\mathfrak{F}=0, \mathfrak{G}=0$ ) (ver Apêndice A2)

$$
\begin{aligned}
& \Pi_{\mu \tau}(x, y)=\int \mathrm{d}^{4} z\left\{\mathfrak{L}_{\mathfrak{F}}\left(\eta_{\mu \tau} \eta_{\alpha \beta}-\eta_{\mu \beta} \eta_{\alpha \tau}\right)\right. \\
& \left.+\mathfrak{L}_{\mathfrak{G}} \epsilon_{\alpha \mu \beta \tau}\right\} \frac{\partial \delta^{4}(x-z)}{\partial z_{\alpha}} \frac{\partial \delta^{4}(y-z)}{\partial z_{\beta}}, \\
& \Pi_{\mu \tau \sigma}(x, y, u)=0,
\end{aligned}
$$

Podemos integrar para obter o tensor de polarização

$$
\Pi_{\mu \tau}(x, y)=-\left[\mathfrak{L}_{\mathfrak{F}}\left(\eta_{\mu \tau} \eta_{\alpha \beta}-\eta_{\mu \beta} \eta_{\alpha \tau}\right)+\mathfrak{L}_{\mathfrak{G}} \epsilon_{\alpha \mu \beta \tau}\right] \frac{\partial}{\partial x_{\alpha}} \frac{\partial \delta^{4}(y-x)}{\partial x_{\beta}} .
$$

Para o limite de ausência de campo externo, $\mathfrak{L}_{\mathfrak{F}}=\mathfrak{L}_{\mathfrak{G}}=0$, graças ao princípio da correspondência, que prevê que no limite infravermelho, as equações de Maxwell padrões devem ser exatas, i.e., não 
estão sujeitas a quaisquer correções. Portanto a resposta não linear é zero

$$
\int \mathrm{d}^{4} y \Pi_{\mu \tau}(x, y) a^{\tau}(y)=0 .
$$

\subsection{Corrente não linearmente induzida}

A resposta não linear (3.7) é devida ao tensor de quarta ordem (veja sua forma explícita no Apêndice A2). Ela pode ser escrita como

$$
\begin{aligned}
& \Pi_{\mu \tau \sigma \rho}(x, y, u, v)=\left.\frac{\delta^{4} \Gamma}{\delta A^{\mu}(x) \delta A^{\tau}(y) \delta A^{\sigma}(u) \delta A^{\rho}(v)}\right|_{\mathfrak{F}=0, \mathfrak{G}=0}= \\
& =\int \mathrm{d}^{4} z\left\{\mathcal{P}_{\alpha \beta \gamma \lambda \mu \tau \sigma \rho} \frac{\partial \delta^{4}(x-z)}{\partial z_{\alpha}} \frac{\partial \delta^{4}(y-z)}{\partial z_{\beta}} \frac{\partial \delta^{4}(u-z)}{\partial z_{\gamma}} \frac{\partial \delta^{4}(z-v)}{\partial z_{\lambda}}\right\},
\end{aligned}
$$

onde definimos o tensor constante $\mathcal{P}_{\alpha \beta \gamma \lambda \mu \tau \sigma \rho}$

$$
\begin{aligned}
& \mathcal{P}_{\alpha \beta \gamma \lambda \mu \tau \sigma \rho}=\mathfrak{L}_{\mathfrak{F F}}\left[\left(\eta_{\alpha \lambda} \eta_{\rho \mu}-\eta_{\mu \lambda} \eta_{\alpha \rho}\right)\left(\eta_{\tau \sigma} \eta_{\beta \gamma}-\eta_{\beta \sigma} \eta_{\tau \gamma}\right)\right. \\
& \left.+\left(\eta_{\beta \lambda} \eta_{\rho \tau}-\eta_{\tau \lambda} \eta_{\beta \rho}\right)\left(\eta_{\mu \sigma} \eta_{\alpha \gamma}-\eta_{\alpha \sigma} \eta_{\mu \gamma}\right)+\left(\eta_{\gamma \lambda} \eta_{\rho \sigma}-\eta_{\sigma \lambda} \eta_{\gamma \rho}\right)\left(\eta_{\mu \tau} \eta_{\alpha \beta}-\eta_{\mu \beta} \eta_{\alpha \tau}\right)\right] \\
& +\mathfrak{L}_{\mathfrak{G G} \mathfrak{G}}\left(\epsilon_{\alpha \mu \beta \tau} \epsilon_{\lambda \rho \gamma \sigma}+\epsilon_{\lambda \rho \alpha \mu} \epsilon_{\beta \tau \gamma \sigma}+\epsilon_{\lambda \rho \beta \tau} \epsilon_{\alpha \mu \gamma \sigma}\right) .
\end{aligned}
$$

Integrando (5.3) por partes

$$
\Pi_{\mu \tau \sigma \rho}(x, y, u, v)=-\mathcal{P}_{\alpha \beta \gamma \lambda \mu \tau \sigma \rho} \frac{\partial}{\partial x_{\alpha}}\left\{\left[\frac{\partial \delta^{4}(y-x)}{\partial x_{\beta}}\right]\left[\frac{\partial \delta^{4}(u-x)}{\partial x_{\gamma}}\right]\left[\frac{\partial \delta^{4}(x-v)}{\partial x_{\lambda}}\right]\right\} .
$$

Vamos definir o potencial linear $a_{\text {lin }}^{\lambda}(x)$ como sendo aquele que satisfaz as equações de Maxwell lineares, cuja fonte é a corrente $j_{\mu}(x)$, i.e.,

$$
j_{\mu}(x)=\left(\square \eta_{\mu \tau}-\partial_{\mu} \partial_{\tau}\right) a_{\operatorname{lin}}^{\lambda}(x) .
$$

Mostramos que o tensor de terceira ordem é nulo na ausência de campos externos (5.1). Portanto, a primeira importante correção (3.12) é devida ao tensor de quarta ordem, onde a corrente não linearmente induzida é dada por

$$
j_{\mu}^{\mathrm{nl}}(x)=-\frac{1}{6} \int \mathrm{d}^{4} y \mathrm{~d}^{4} u \mathrm{~d}^{4} v \Pi_{\mu \tau \sigma \rho}(x, y, u, v) a^{\tau}(y) a^{\sigma}(u) a^{\rho}(v)
$$

Logo, usando (5.5)

$$
\begin{array}{r}
j_{\mu}^{\mathrm{nl}}(x)=\frac{\mathcal{P}_{\alpha \beta \gamma \lambda \mu \tau \sigma \rho}}{6} \frac{\partial}{\partial x_{\alpha}}\left[\int \frac{\partial \delta^{4}(y-x)}{\partial x_{\beta}} a^{\tau}(y) \mathrm{d}^{4} y \int \frac{\partial \delta^{4}(u-x)}{\partial x_{\gamma}} a^{\sigma}(u) \mathrm{d}^{4} u\right. \\
\left.\quad \times \int \frac{\partial \delta^{4}(x-v)}{\partial x_{\lambda}} a^{\rho}(v) \mathrm{d}^{4} v\right]=\frac{\mathcal{P}_{\alpha \beta \gamma \lambda \mu \tau \sigma \rho}}{6} \frac{\partial}{\partial x_{\alpha}}\left[\frac{\partial a^{\tau}(x)}{\partial x_{\beta}} \frac{\partial a^{\sigma}(x)}{\partial x_{\gamma}} \frac{\partial a^{\rho}(x)}{\partial x_{\lambda}}\right]
\end{array}
$$

e definindo $f_{\beta \tau}(x)$ como o dobro da parte antissimétrica de $\frac{\partial a^{\tau}(x)}{\partial x_{\beta}}$, i.e., $f^{\beta \tau}(x)=\frac{\partial a^{\tau}(x)}{\partial x_{\beta}}-\frac{\partial a^{\beta}(x)}{\partial x_{\tau}}$, notando que a parte simétrica de $\frac{\partial a^{\tau}(x)}{\partial x_{\beta}}$ se torna zero após a contração com o tensor $\mathcal{P}_{\alpha \beta \gamma \lambda \mu \tau \sigma \rho}$ 
(porque ele é antissimétrico com respeito aos respectivos pares de índices: $\beta \Leftrightarrow \tau, \gamma \Leftrightarrow \sigma$ e $\lambda \Leftrightarrow \rho$ ), obtemos

$$
j_{\mu}^{\mathrm{nl}}(x)=\frac{\mathcal{P}_{\alpha \beta \gamma \lambda \mu \tau \sigma \rho}}{48} \frac{\partial}{\partial x_{\alpha}}\left[f^{\beta \tau}(x) f^{\gamma \sigma}(x) f^{\lambda \rho}(x)\right] .
$$

E, por (5.4), a corrente não linearmente induzida é dada por

$$
j_{\mu}^{\mathrm{nl}}(x)=\frac{1}{4} \mathfrak{L}_{\mathfrak{F} \mathfrak{F}} \frac{\partial}{\partial x_{\alpha}}\left[f_{\alpha \mu}(x) f_{\beta \gamma}(x) f^{\beta \gamma}(x)\right]+\frac{1}{4} \mathfrak{L}_{\mathfrak{G} \mathfrak{G}} \frac{\partial}{\partial x_{\alpha}}\left[\tilde{f}_{\alpha \mu}(x) f_{\beta \gamma}(x) \tilde{f}^{\beta \gamma}(x)\right] .
$$

Em termos dos campos explícitos $\mathbf{E}=\mathbf{E}(x)$ e $\mathbf{B}=\mathbf{B}(x)$, definidos por $E_{i}(x)=f_{0 i}(x)$, e $B_{i}(x)=$ $\tilde{f}_{0 i}(x)=\frac{1}{2} \epsilon_{i j k} f^{j k}(x)$, e tendo em mente que $f_{\beta \gamma}(x) f^{\beta \gamma}(x)=2\left(\mathbf{B}^{2}(x)-\mathbf{E}^{2}(x)\right)$ e $f_{\beta \gamma}(x) \tilde{f}^{\beta \gamma}(x)=$ $-4(\mathbf{E}(x) \cdot \mathbf{B}(x))$

$$
j_{\mu}^{\mathrm{nl}}(x)=\frac{1}{2} \mathfrak{L}_{\mathfrak{F} \mathfrak{F}} \frac{\partial}{\partial x_{\alpha}}\left[f_{\alpha \mu}(x)\left(\mathbf{B}^{2}(x)-\mathbf{E}^{2}(x)\right)\right]-\mathfrak{L}_{\mathfrak{G} \mathfrak{G}} \frac{\partial}{\partial x_{\alpha}}\left[\tilde{f}_{\alpha \mu}(x)(\mathbf{E}(x) \cdot \mathbf{B}(x))\right] .
$$

Definindo $j_{\mu}^{\mathrm{nl}}(x)=\left(j_{0}^{\mathrm{nl}}(x),-\mathbf{j}^{\mathrm{nl}}(x)\right)$, podemos obter a corrente geral em termos do campo eletromagnético

$$
\begin{aligned}
& j_{0}^{\mathrm{nl}}(x)=\frac{1}{2} \mathfrak{L}_{\mathfrak{F} \mathfrak{F}} \boldsymbol{\nabla} \cdot\left[\left(\mathbf{B}^{2}-\mathbf{E}^{2}\right) \mathbf{E}\right]-\mathfrak{L}_{\mathfrak{G G}} \boldsymbol{\nabla} \cdot[(\mathbf{E} \cdot \mathbf{B}) \mathbf{B}], \\
& \mathbf{j}^{\mathrm{nl}}(x)=-\frac{1}{2} \mathfrak{L}_{\mathfrak{F} \mathfrak{F}}\left(\frac{\partial}{\partial t}\left[\left(\mathbf{B}^{2}-\mathbf{E}^{2}\right) \mathbf{E}\right]+\boldsymbol{\nabla} \times\left[\left(\mathbf{B}^{2}-\mathbf{E}^{2}\right) \mathbf{B}\right]\right) \\
& +\mathfrak{L}_{\mathfrak{G G}}\left(\frac{\partial}{\partial t}[(\mathbf{E} \cdot \mathbf{B}) \mathbf{B}]+\boldsymbol{\nabla} \times[(\mathbf{E} \cdot \mathbf{B}) \mathbf{E}]\right) .
\end{aligned}
$$

Note que corrente $j^{\mathrm{nl}}(x)$ se conserva, pois satisfaz a equação de continuidade $\partial^{\mu} j_{\mu}^{\mathrm{nl}}(x)=\boldsymbol{\nabla}$. $\mathrm{j}^{\mathrm{nl}}(x)+\frac{\partial}{\partial t} j_{0}^{\mathrm{nl}}(x)=0$, fato decorrente diretamente da transversalidade do tensor de polarização (3.9), e assim podemos atribuir significado físico a essa corrente não linear, e, de fato, tratar como uma (densidade de) corrente. 


\section{Capítulo 6}

\section{Equações de Maxwell não lineares perturbativas e estacionárias}

Agora podemos considerar teoria de perturbação de primeira ordem para obter a primeira correção não linear para o campo $a^{\lambda}(x)$. Chamemos esta correção de $a_{(1)}^{\lambda}(x)$. De (3.4) e (5.6), a aproximação de primeira ordem para o campo é dada por $a_{(0)}^{\lambda}(x)=a_{\operatorname{lin}}^{\lambda}(x)=\int d^{4} y D^{\lambda \rho}(x-y) j_{\rho}(y)$. A perturbação é obtida por iteração deste campo, substituindo o campo em (5.7), i.e.,

$$
\begin{aligned}
j_{\rho}^{\mathrm{nl}(0)}(x)= & \frac{1}{6} \int \mathrm{d}^{4} y \mathrm{~d}^{4} u \mathrm{~d}^{4} v \Pi_{\mu \tau \sigma \rho}(x, y, u, v) a_{(0)}^{\tau}(y) a_{(0)}^{\sigma}(u) a_{(0)}^{\rho}(v), \\
& {\left[\square \eta_{\mu \tau}-\partial_{\mu} \partial_{\tau}\right] a_{(1)}^{\tau}(x)=j_{\mu}(x)+j_{\mu}^{\mathrm{nl}(0)}(x) . }
\end{aligned}
$$

Dividimos o campo em duas partes, o campo linear $a_{\text {lin }}^{\lambda}(x)$ e o campo $a_{\mathrm{nl}}^{\lambda}(x)$ devido à corrente não linearmente induzida:

$$
\begin{aligned}
& a_{(1)}^{\lambda}(x)=a_{\operatorname{lin}}^{\lambda}(x)+a_{\mathrm{nl}}^{\lambda}(x), \\
& a_{\operatorname{lin}}^{\lambda}(x)=a_{(0)}^{\lambda}(x) \\
& a_{\mathrm{nl}}^{\lambda}(x)=\int d^{4} y D^{\lambda \rho}(x-y) j_{\rho}^{\mathrm{nl}(0)}(y) .
\end{aligned}
$$

Queremos solucionar (6.1) impondo independência temporal. Para o campo linear $a_{\text {lin }}^{\lambda}(x)$, resolvemos as equações de Maxwell lineares. Para o campo não linear, definimos os potenciais não lineares e suas derivadas do campo eletromagnético

$$
\begin{aligned}
& \mathbf{E}_{\mathrm{nl}}(\mathbf{r})=\nabla a_{\mathrm{nl}}^{0}(\mathbf{r}), \\
& \mathbf{B}_{\mathrm{nl}}(\mathbf{r})=\nabla \times \mathbf{a}_{\mathrm{nl}}(\mathbf{r}) .
\end{aligned}
$$

Agora, usando as propriedades do propagador (3.10), notamos que o campo elétrico não linear $\mathbf{E}_{\mathrm{nl}}(\mathbf{r})$ e o campo magnético não linear $\mathbf{B}_{\mathrm{nl}}(\mathbf{r})$ são as soluções para as seguintes equações de Maxwell com fonte estacionária $j_{\mu}^{\mathrm{nl}}(x)$

$$
\begin{aligned}
\nabla \cdot \mathbf{E}_{\mathrm{nl}}(\mathbf{r}) & =j_{0}^{\mathrm{nl}}(\mathbf{r}), \\
\nabla \times \mathbf{E}_{\mathrm{nl}}(\mathbf{r}) & =\mathbf{0} \\
\nabla \cdot \mathbf{B}_{\mathrm{nl}}(\mathbf{r}) & =0 \\
\nabla \times \mathbf{B}_{\mathrm{nl}}(\mathbf{r}) & =\mathbf{j}^{\mathrm{nl}}(\mathbf{r})
\end{aligned}
$$


A unicidade das soluções $\mathbf{E}_{\mathrm{nl}}(\mathbf{r})$ e $\mathbf{B}_{\mathrm{nl}}(\mathbf{r})$ podem ser verificadas no Apêndice $\mathrm{C}$, se impormos

$$
\lim _{r \rightarrow \infty} r \mathbf{E}_{\mathrm{nl}}(\mathbf{r})=\lim _{r \rightarrow \infty} r \mathbf{B}_{\mathrm{nl}}(\mathbf{r})=0 .
$$

Por (6.1) e (6.2), e pela definição dos potenciais eletromagnéticos estacionários no regime linear $\mathbf{E}_{\text {lin }}(\mathbf{r})=\nabla a_{\text {lin }}^{0}(\mathbf{r})$, e $\mathbf{B}_{\text {lin }}(\mathbf{r})=\boldsymbol{\nabla} \times \mathbf{a}_{\text {lin }}(\mathbf{r})$, os campos corrigidos $\mathbf{E}(\mathbf{r})$ e $\mathbf{B}(\mathbf{r})$ são dados por:

$$
\begin{aligned}
& \mathbf{E}(\mathbf{r})=\mathbf{E}_{\text {lin }}(\mathbf{r})+\mathbf{E}_{\mathrm{nl}}(\mathbf{r}) \\
& \mathbf{B}(\mathbf{r})=\mathbf{B}_{\text {lin }}(\mathbf{r})+\mathbf{B}_{\mathrm{nl}}(\mathbf{r}) .
\end{aligned}
$$

\subsection{Soluções das equações inomogêneas}

Para o problema eletrostático, i.e., definindo $\mathbf{j}^{\mathrm{nl}}(\mathbf{r})=0$, começamos com a correção do campo elétrico não linear, devido ao tensor de polarização de quarta ordem, e assim encontramos a densidade de carga não linearmente induzida, por (5.9):

$$
4 \pi j_{0}^{\mathrm{nl}}(\mathbf{r})=-\frac{1}{2} \mathfrak{L}_{\mathfrak{F} \mathfrak{F}} \boldsymbol{\nabla} \cdot\left[\mathbf{E}_{\text {lin }}(\mathbf{r}) E_{\text {lin }}^{2}(\mathbf{r})\right] .
$$

Usando a primeira equação de (6.3), podemos expressar o divergente na forma vetorial

$$
\nabla \cdot\left[\mathbf{E}_{\mathrm{nl}}(\mathbf{r})+\frac{1}{2} \mathfrak{L}_{\mathfrak{F} \mathfrak{F}} \mathbf{E}_{\text {lin }}(\mathbf{r}) E_{\text {lin }}^{2}(\mathbf{r})\right]=0
$$

Para a solução geral da equação acima, podemos considerar um certo campo vetorial $\boldsymbol{\Omega}(\mathbf{r})$, tal que

$$
\mathbf{E}_{\text {lin }}(\mathbf{r})=-\frac{1}{2} \mathfrak{L}_{\mathfrak{F} \mathfrak{F}} \mathbf{E}_{\text {lin }}(\mathbf{r}) E_{\text {lin }}^{2}(\mathbf{r})+[\boldsymbol{\nabla} \times \boldsymbol{\Omega}(\mathbf{r})] .
$$

A segunda equação de (6.3) nos fornece a outra equação, que fixa o campo $\boldsymbol{\Omega}(\mathbf{r})$.

Podemos calcular o campo magnético da mesma forma. Neste caso, dada uma corrente que produz um campo magnético linear, agora definindo $j_{0}^{\mathrm{nl}}(\mathbf{r})=0$, a corrente nao linear é calculada devida a (5.9)

$$
\mathbf{j}^{\mathrm{nl}}(\mathbf{r})=-\frac{1}{2} \mathfrak{L}_{\mathfrak{F} \mathfrak{F}} \boldsymbol{\nabla} \times\left[\mathbf{B}_{\text {lin }}(\mathbf{r}) B_{\operatorname{lin}}^{2}(\mathbf{r})\right] .
$$

Podemos usar a quarta equação de (6.3), para o campo magnético, para encontrar

$$
\nabla \times\left[\mathbf{B}_{\mathrm{nl}}(\mathbf{r})+\frac{1}{2} \mathfrak{L}_{\mathfrak{F F}} \mathbf{B}_{\text {lin }}(\mathbf{r}) B_{\text {lin }}^{2}(\mathbf{r})\right]=0 .
$$

Pela mesma razão do caso eletrostático, definimos um certo campo escalar $\Phi(\mathbf{r})$, tal que

$$
\mathbf{B}_{\mathrm{nl}}(\mathbf{r})=-\frac{1}{2} \mathfrak{L}_{\mathfrak{F} \mathfrak{F}} \mathbf{B}_{\text {lin }}(\mathbf{r}) B_{\text {lin }}^{2}(\mathbf{r})+\nabla \Phi(\mathbf{r}) .
$$

Analogamente, mutatis mutandis, a terceira equação de (6.3), nos fornece a outra equação, que fixa $\Omega(\mathbf{r})$. 


\subsection{Soluções das equações homogêneas}

Podemos obter o resultado geral de (6.7), para o caso $\mathbf{j}^{\mathrm{nl}}(\mathbf{r})=0$. Definindo o campo

$$
\mathcal{E}(\mathbf{r})=-\frac{1}{2} \mathfrak{L}_{\mathfrak{F} \mathfrak{F}} \mathbf{E}_{\operatorname{lin}}(\mathbf{r}) E_{\operatorname{lin}}^{2}(\mathbf{r}) .
$$

Portanto,

$$
\mathcal{E}(\mathbf{r})=\mathbf{E}_{\mathrm{nl}}(\mathbf{r})-[\boldsymbol{\nabla} \times \boldsymbol{\Omega}(\mathbf{r})] .
$$

Pela segunda equação de (6.3)

$$
[\boldsymbol{\nabla} \times[\boldsymbol{\nabla} \times \boldsymbol{\Omega}(\mathbf{r})]]=-[\boldsymbol{\nabla} \times \mathcal{E}(\mathbf{r})] .
$$

O campo vetorial $\Omega(\mathbf{r})$ deve satisfazer a seguinte equação de Poisson

$$
-\boldsymbol{\nabla}^{2} \boldsymbol{\Omega}(\mathbf{r})+\boldsymbol{\nabla}(\boldsymbol{\nabla} \cdot \boldsymbol{\Omega}(\mathbf{r}))=-[\boldsymbol{\nabla} \times \mathcal{E}(\mathbf{r})] .
$$

Podemos escolher $\boldsymbol{\nabla} \cdot \boldsymbol{\Omega}=0$ como uma escolha de calibre: Definindo a transformação de calibre $\boldsymbol{\Omega}^{\prime}(\mathbf{r})=\boldsymbol{\Omega}(\mathbf{r})+\nabla \lambda(\mathbf{r})$, onde $\lambda(\mathbf{r})$ é alguma função analítica, cujo gradiente não altera o rotacional, i.e., $\left[\boldsymbol{\nabla} \times \boldsymbol{\Omega}^{\prime}(\mathbf{r})\right]=[\boldsymbol{\nabla} \times \boldsymbol{\Omega}(\mathbf{r})]+[\boldsymbol{\nabla} \times \boldsymbol{\nabla} \lambda(\mathbf{r})]=[\boldsymbol{\nabla} \times \boldsymbol{\Omega}(\mathbf{r})]$. Encontremos a função $\lambda(\mathbf{r})$, tal que $\nabla \cdot \Omega^{\prime}(\mathbf{r})=0$ :

$$
\boldsymbol{\Omega}^{\prime}=\boldsymbol{\Omega}+\boldsymbol{\nabla} \lambda \rightarrow \nabla \cdot \boldsymbol{\Omega}^{\prime}=\boldsymbol{\nabla} \cdot \boldsymbol{\Omega}+\boldsymbol{\nabla} \cdot \boldsymbol{\nabla} \lambda=0
$$

Portanto, $\lambda(\mathbf{r})$ está sujeito à equação de Poisson

$$
\nabla^{2} \lambda(\mathbf{r})=-\nabla \cdot \Omega(\mathbf{r}) .
$$

Logo,

$$
\boldsymbol{\nabla}^{2} \boldsymbol{\Omega}(\mathbf{r})=[\boldsymbol{\nabla} \times \mathcal{E}(\mathbf{r})] .
$$

Assim, cada componente de $\boldsymbol{\Omega}(\mathbf{r})$ satisfaz a equação de Poisson acima, bem como obriga o campo elétrico $\mathbf{E}_{\mathrm{nl}}(\mathbf{r})$ a obedecer a condição $\boldsymbol{\nabla} \times \mathbf{E}_{\mathrm{nl}}(\mathbf{r})=\mathbf{0}$.

Então, tendo em mente (6.11), $\mathbf{E}_{\mathrm{nl}}(\mathbf{r})$ é a projeção longitudinal do campo $\mathcal{E}(\mathbf{r})$, i.e.,

$$
E_{\mathrm{nl}}^{i}(\mathbf{r})=\frac{\nabla_{i} \nabla_{j}}{\nabla^{2}} \mathcal{E}_{j}(\mathbf{r})=-\frac{\nabla_{i} \nabla_{j}}{4 \pi} \int \frac{\mathcal{E}_{j}\left(\mathbf{r}^{\prime}\right)}{\left|\mathbf{r}-\mathbf{r}^{\prime}\right|} \mathrm{d} \mathbf{r}^{\prime},
$$

ou, na forma vetorial (Apêndice C1), se torna

$$
\mathbf{E}_{\mathrm{nl}}(\mathbf{r})=-\frac{1}{4 \pi} \boldsymbol{\nabla} \int \frac{\boldsymbol{\nabla}^{\prime} \cdot \mathcal{E}\left(\mathbf{r}^{\prime}\right)}{\left|\mathbf{r}-\mathbf{r}^{\prime}\right|} \mathrm{d} \mathbf{r}^{\prime} .
$$

Note que a substituição do campo de uma carga puntiforme em (6.13) através de (6.10) causaria a divergência na integral (6.13) próximo da origem $r=0:$ a presente aproximação falha perto da carga, pôsto que ela não é verificada para campos muito intensos. Lidar com cargas puntiformes requer ir além da aproximação infra-vermelho seguida nesta seção. Não obstante, (6.13) é aplicável a cargas extensas.

Podemos definir o potencial escalar não linear por

$$
a_{\mathrm{nl}}^{0}(\mathbf{r})=\frac{1}{4 \pi} \int \frac{\nabla^{\prime} \cdot \mathcal{E}\left(\mathbf{r}^{\prime}\right)}{\left|\mathbf{r}-\mathbf{r}^{\prime}\right|} \mathrm{d} \mathbf{r}^{\prime},
$$


e podemos comparar a (6.1).

Analogamente, mutatis mutandis, para o caso magnetostático, i.e., definindo novamente $j_{0}^{\mathrm{nl}}(\mathbf{r})=$ 0 , podemos calcular o campo magnético. Definindo

$$
\mathfrak{h}(\mathbf{r})=-\frac{1}{2} \mathfrak{L}_{\mathfrak{F} \mathfrak{F}} \mathbf{B}_{\operatorname{lin}}(\mathbf{r}) B_{\operatorname{lin}}^{2}(\mathbf{r}),
$$

logo:

$$
\mathfrak{h}(\mathbf{r})=\mathbf{B}_{\mathrm{nl}}(\mathbf{r})-\nabla \Phi(\mathbf{r})
$$

Pela terceira equação de (6.3)

$$
\boldsymbol{\nabla} \cdot \mathbf{B}_{\mathrm{nl}}(\mathbf{r})=\boldsymbol{\nabla} \cdot \mathfrak{h}(\mathbf{r})+\nabla^{2} \Phi(\mathbf{r})=0 .
$$

Portanto, como o caso elétrico, o campo escalar $\Phi(\mathbf{r})$ deve satisfazer a seguinte equação de Poisson

$$
\nabla^{2} \Phi(\mathbf{r})=-\nabla \cdot \mathfrak{h}(\mathbf{r}) .
$$

Assim, podemos encontrar o campo magnético $\mathbf{B}_{\mathrm{nl}}(\mathbf{r})$, tendo em mente (6.16), como sendo a projeção transversal do campo $\mathfrak{h}(\mathbf{r})$, i.e.,

$$
B_{\mathrm{nl}}^{i}(\mathbf{r})=\left(\delta_{i j}-\frac{\nabla_{i} \nabla_{j}}{\nabla^{2}}\right) \mathfrak{h}_{j}(\mathbf{r})=\mathfrak{h}_{i}(\mathbf{r})+\frac{\nabla_{i} \nabla_{j}}{4 \pi} \int \frac{\mathfrak{h}_{j}\left(\mathbf{r}^{\prime}\right)}{\left|\mathbf{r}-\mathbf{r}^{\prime}\right|} \mathrm{d} \mathbf{r}^{\prime} .
$$

ou, na forma vetorial (Apêndice C2),

$$
\mathbf{B}_{\mathrm{nl}}(\mathbf{r})=\mathfrak{h}(\mathbf{r})+\frac{1}{4 \pi} \boldsymbol{\nabla} \int \frac{\boldsymbol{\nabla}^{\prime} \cdot \mathfrak{h}\left(\mathbf{r}^{\prime}\right)}{\left|\mathbf{r}-\mathbf{r}^{\prime}\right|} \mathrm{d} \mathbf{r}^{\prime}=\frac{1}{4 \pi} \boldsymbol{\nabla} \times \int \frac{\boldsymbol{\nabla}^{\prime} \times \mathfrak{h}\left(\mathbf{r}^{\prime}\right)}{\left|\mathbf{r}-\mathbf{r}^{\prime}\right|} \mathrm{d} \mathbf{r}^{\prime} .
$$

Novamente, podemos definir o potencial vetor não linear

$$
\mathbf{a}_{\mathrm{nl}}(\mathbf{r})=\frac{1}{4 \pi} \int \frac{\nabla^{\prime} \times \mathfrak{h}\left(\mathbf{r}^{\prime}\right)}{\left|\mathbf{r}-\mathbf{r}^{\prime}\right|} \mathrm{d} \mathbf{r}^{\prime}
$$

e podemos comparar a (6.1).

\subsection{Magnetização, polarização e campos auxiliares não line- ares}

No caso magnetostático $\left(j_{0}^{\mathrm{nl}}(\mathbf{r})=0\right)$, na ausência de correntes livres, como no regime linear $\boldsymbol{\nabla} \times$ $\mathbf{B}_{\text {lin }}(\mathbf{r})=\mathbf{j}(\mathbf{r})=\boldsymbol{\nabla} \times \mathbf{M}(\mathbf{r})$ [17], convém definir a generalização não linear da magnetização $\mathbf{M}_{\mathrm{nl}}(\mathbf{r})$, devido a (6.8), i.e.,

$$
\mathbf{M}_{\mathrm{nl}}(\mathbf{r})=\mathfrak{h}(\mathbf{r})=-\frac{1}{2} \mathfrak{L}_{\mathfrak{F F}} \mathbf{B}_{\text {lin }}(\mathbf{r}) B_{\operatorname{lin}}^{2}(\mathbf{r}) .
$$

Por analogia com o campo magnético auxiliar linear $\mathbf{H}(\mathbf{r})$, definimos $\mathbf{H}^{\mathrm{nl}}(\mathbf{r})$ como solução das seguintes equações

$$
\begin{aligned}
\boldsymbol{\nabla} \times \mathbf{H}_{\mathrm{nl}}(\mathbf{r}) & =0, \\
\boldsymbol{\nabla} \cdot \mathbf{H}_{\mathrm{nl}}(\mathbf{r}) & =-\boldsymbol{\nabla} \cdot \mathbf{M}^{\mathrm{nl}}(\mathbf{r}),
\end{aligned}
$$


que são satisfeitas por:

$$
\mathbf{H}_{\mathrm{nl}}(\mathbf{r})=\frac{1}{4 \pi} \boldsymbol{\nabla} \int \frac{\boldsymbol{\nabla}^{\prime} \cdot \mathbf{M}_{\mathrm{nl}}\left(\mathbf{r}^{\prime}\right)}{\left|\mathbf{r}-\mathbf{r}^{\prime}\right|} \mathrm{d} \mathbf{r}^{\prime} .
$$

Imediatamente reconhecemos que o campo magnético é a projeção transversal da magnetização, e o campo magnético auxiliar, com o sinal trocado, é a projeção longitudinal da magnetização, i.e., $\mathbf{B}_{\mathrm{nl}}(\mathbf{r})=\mathbf{H}_{\mathrm{nl}}(\mathbf{r})+\mathbf{M}_{\mathrm{nl}}(\mathbf{r})$.

$\mathrm{O}$ caso eletrostático $\left(\mathbf{j}_{\mathrm{nl}}(\mathbf{r})=\mathbf{0}\right)$ é muito similar, tendo em mente que, quando não há cargas livres, $\boldsymbol{\nabla} \cdot \mathbf{E}_{\text {lin }}(\mathbf{r})=j_{0}(\mathbf{r})=-\boldsymbol{\nabla} \cdot \mathbf{P}(\mathbf{r})$. A partir de (6.6), definimos a polarização não linear $\mathbf{P}_{\mathrm{nl}}(\mathbf{r})$ por

$$
\mathbf{P}_{\mathrm{nl}}(\mathbf{r})=-\mathcal{E}(\mathbf{r})=\frac{1}{2} \mathfrak{L}_{\mathfrak{F} \mathfrak{F}} \mathbf{E}_{\text {lin }}(\mathbf{r}) E_{\text {lin }}^{2}(\mathbf{r})
$$

Podemos definir o campo elétrico auxiliar $\mathbf{D}_{\mathrm{nl}}(\mathbf{r})$, que satisfaz, na ausência de cargas livres, as equações análogas ao campo elétrico auxiliar $\mathbf{D}(\mathbf{r})$

$$
\begin{aligned}
\nabla \times \mathbf{D}_{\mathrm{nl}}(\mathbf{r}) & =\boldsymbol{\nabla} \times \mathbf{P}_{\mathrm{nl}}(\mathbf{r}) \\
\boldsymbol{\nabla} \cdot \mathbf{D}_{\mathrm{nl}}(\mathbf{r}) & =0
\end{aligned}
$$

Portanto,

$$
\mathbf{D}_{\mathrm{nl}}(\mathbf{r})=\mathbf{P}_{\mathrm{nl}}(\mathbf{r})+\frac{1}{4 \pi} \boldsymbol{\nabla} \int \frac{\boldsymbol{\nabla}^{\prime} \cdot \mathbf{P}_{\mathrm{nl}}\left(\mathbf{r}^{\prime}\right)}{\left|\mathbf{r}-\mathbf{r}^{\prime}\right|} \mathrm{d} \mathbf{r}^{\prime} .
$$

Como no caso linear, o campo elétrico, com o sinal trocado, é a projeção longitudinal da polarização, e o campo elétrico auxiliar é a projeção transversal da polarização, i.e., $\mathbf{D}_{\mathrm{nl}}(\mathbf{r})=\mathbf{E}_{\mathrm{nl}}(\mathbf{r})+\mathbf{P}_{\mathrm{nl}}(\mathbf{r})$.

Suponha que estes campos auxiliares obedeçam à condição

$$
\lim _{r \rightarrow \infty} r \mathbf{H}_{\mathrm{nl}}(\mathbf{r})=\lim _{r \rightarrow \infty} r \mathbf{D}_{\mathrm{nl}}(\mathbf{r})=0
$$

Então estas soluções são, como no caso (6.4), únicas (veja demonstração no Apêndice D). 


\section{Capítulo 7}

\section{Aplicações a correntes estacionárias}

Neste capítulo, encontramos algumas aplicações, como a correção não linear dos campos devido a uma esfera, um fio infinito e um plano infinito, carregados eletricamente, uma esfera magnetizada e uam esfera polarizada. Mostramos que o dipolo possui uma correção mais importante que a do monopolo no que se refere ao comportamento a longas distâncias, e modelamos estrelas de quarks, pulsares e alguns bárions.

\subsection{Correções para monopolos eletrostáticos}

Os casos especiais de monopolos eletrostáticos possuem a propriedade que o operador de projeção longitudinal $\nabla_{i} \nabla_{j} \Delta^{-1}$ atua no campo $\mathcal{E}_{i}(\mathbf{r})$ como o operador identidade $\delta_{i j}$. Devido a esse fato, o campo elétrico não linear é o próprio campo $\mathcal{E}(r)=-\frac{1}{2} \mathfrak{L}_{\mathfrak{F} \mathfrak{F}} \mathbf{E}_{\text {lin }}(\mathbf{r}) E_{\text {lin }}^{2}(\mathbf{r})$. Tendo a expressão para o campo elétrico em mãos, podemos modelar e calcular o campo gerado por corpos fortemente carregados, como uma estrela hipotética existente no universo: a estrela de quarks.

\subsubsection{Simetria esférica: A esfera carregada}

Considere uma esfera com carga total $q$ em repouso na origem de um sistema de coordenadas ${ }^{1}$. Se o raio da carga não é muito pequeno (o campo deve variar lentamente, por hipótese da aproximação infravermelho), pode-se descrever o campo produzido pelo núcleo de alguns átomos não tão pesados. O campo elétrico externo é dado por

$$
\mathbf{E}_{\mathrm{lin}}(\mathbf{r})=\frac{q}{4 \pi r^{2}} \mathbf{e}_{r}
$$

onde $\mathbf{e}_{r}$ é o vetor unitário ao longo da direção radial. Portanto, $\mathcal{E}(r)$ possui a forma $\mathcal{E}_{i}(\mathbf{r})=x_{i} \Lambda(r)$, onde $\Lambda$ é uma função escalar que depende somente da distância $r=|\mathbf{r}|$ e por diferenciação direta, observamos que

$$
\nabla_{i} \nabla_{j}\left(x_{j} \Lambda(r)\right)=\nabla^{2} x_{i} \Lambda(r)
$$

i.e., esta projeção atua como o operador identidade: $\frac{\nabla_{i} \boldsymbol{\nabla}_{j}}{\nabla^{2}}=\delta_{i j}$.

Por (6.13) e (7.2) os campo elétrico não linear é dado por $\mathbf{E}_{\mathrm{nl}}(\mathbf{r})=\mathcal{E}(r)=-\frac{1}{2} \mathfrak{L}_{\mathfrak{F} \mathfrak{F}} E_{\text {lin }}^{2}(r) \mathbf{E}_{\text {lin }}(r)$. Como resultado, a correção não linear para o campo elétrico (6.5) é dada pela soma do campo linear

\footnotetext{
${ }^{1}$ Denotamos o versor radial das coordenadas esféricas por $\mathbf{e}_{r}=\frac{r}{r}$, e o versor zenital denotamos por $\mathbf{e}_{\theta}$.
} 
(7.1) e do campo não linear $(6.10)^{2}$

$$
\mathbf{E}(\mathbf{r})=\mathbf{E}_{\text {lin }}(\mathbf{r})\left(1-\frac{1}{2} \mathfrak{L}_{\mathfrak{F} \mathfrak{F}} E_{\text {lin }}^{2}(r)\right) .
$$

Para a lagrangeana de Euler-Heisenberg [2], $\mathfrak{L}_{\mathfrak{F} \mathfrak{F}}=\frac{4 \alpha e^{2}}{45 \pi m^{4}}$, a lei de Coulomb corrigida é dada por

$$
\mathbf{E}(\mathbf{r})=\mathbf{E}_{\text {lin }}(\mathbf{r})\left(1-\frac{2 \alpha}{45 \pi}\left(\frac{e E_{\text {lin }}(r)}{m^{2}}\right)^{2}\right)
$$

se $E(\mathbf{r})<<\frac{m^{2}}{e}$, o valor crítico de Schwinger. Conforme mencionado antes, se o campo é comparável a esse valor crítico, o vácuo se torna instável, e é necessário levar em conta criação de pares elétronpósitron $[27]$.

Para o campo produzido por um átomo com $q=Z e$ e $Z$ em torno de algumas dezenas, encontramos uma correção não linear típica de $10^{-5}$ à distância equivalente ao comprimento de Compton do elétron.

A equação (7.4) pode ser aplicada a uma carga $q$ esfericamente simétrica, homogeneamente carregada em uma esfera de raio $R$. Definimos duas regiões $r<R$ e $r>R$, e o campo elétrico linear é dado por

$$
\mathbf{E}_{\text {lin }}(r)=\Theta(R-r) \mathbf{E}^{<}(r)+\Theta(r-R) \mathbf{E}^{>}(r),
$$

onde

$$
\mathbf{E}^{<}(r)=\frac{q r}{4 \pi R^{3}} \mathbf{e}_{r}, \quad \mathbf{E}^{>}(r)=\frac{q}{4 \pi r^{2}} \mathbf{e}_{r} .
$$

Substituimos esse campo em (7.4) para modelar monopolos macroscópicos altamente carregados, como as estrelas de quarks. Não há registro oficial da existência de tais tipos de estrelas, mas se existirem, sua alta densidade de carga propicia o tratamento adequado do seu campo elétrico por meio desse modelo simetricamente esférico.

\subsubsection{Simetria cilíndrica com translação: O plano, o fio e a espira infini- tos, e carregados}

Outros três casos, quando as projeções em (6.12) ou em (6.17) se reduzem a identidades triviais.

Um deles é fornecido pela invariância de translação ao longo de todo plano ortogonal a uma dada direção ${ }^{3}$ (direção $\mathbf{e}_{3}$, por exemplo), típico de um plano infinito com uma certa espessura, carregado homogeneamente ao longo das direções $\mathbf{e}_{1}$ e $\mathbf{e}_{2}$. Então $\mathcal{E}_{1,2}(\mathbf{r})=0$, e $\mathcal{E}_{3}(\mathbf{r})=x_{3} \Lambda\left(x_{3}\right)$ satisfaz as condições tais que, quando aplicado ao campo $\mathcal{E}(\mathbf{r})$, o operador de projeção é a identidade: $\frac{\nabla_{i} \nabla_{j}}{\nabla^{2}}=\delta_{i j}$. Logo, ao invés de (7.4) teremos

$$
E_{3}\left(x_{3}\right)=E_{3}^{\operatorname{lin}}\left(x_{3}\right)\left(1-\frac{2 \alpha}{45 \pi}\left(\frac{e E^{\operatorname{lin}}\left(x_{3}\right)}{m^{2}}\right)^{2}\right), \quad E_{1,2}=0 .
$$

Se, além disso, a densidade de carga volumétrica $\kappa$ é também homogênea ao longo do eixo 3 , então finalmente $\kappa=$ const, e o plano esta disposto ao longo do plano tal que $x_{3}=0$, seu campo elétrico

\footnotetext{
${ }^{2}$ Para uma carga não pontual, i.e., com um raio finito $R$, o campo interno também é dado por (7.1), mas neste caso o campo linear é dado por $\mathbf{E}(r)=\frac{q r}{4 \pi R^{3}} \mathbf{e}_{r}$.

${ }^{3}$ Os versores $\mathbf{e}_{1}, \mathbf{e}_{2}$, e $\mathbf{e}_{3}$ denotamos como vetores unitários mutuamente ortogonais, na referência do sistema cartesiano ao longo dos eixos $x_{1}, x_{2}$, e $x_{3}$ respectivamente.
} 
linear

$$
\begin{gathered}
\mathbf{E}^{\operatorname{lin}}\left(x_{3}\right)=\Theta\left(\frac{d}{2}-\left|x_{3}\right|\right) \mathbf{E}^{<}\left(x_{3}\right)+\Theta\left(\left|x_{3}\right|-\frac{d}{2}\right) \mathbf{E}^{>}\left(x_{3}\right), \\
\mathbf{E}^{<}\left(x_{3}\right)=\kappa x_{3} \mathbf{e}_{3}, \quad \mathbf{E}^{>}\left(x_{3}\right)=\frac{\kappa d}{2} \operatorname{sgn}\left(x_{3}\right) \mathbf{e}_{3},
\end{gathered}
$$

onde $d$ é a espessura do plano, deve ser usado em (7.7) para se obter a correção não linear para o campo produzido por esse plano carregado ${ }^{4}$.

Outro caso que trivializa o operador de projeção é a simetria cilíndrica sobre rotações em torno do eixo 3 fornecida pela invariância sobre translações ao longo desse eixo ${ }^{5}$. Então $\mathcal{E}_{3}(\mathbf{r})=0$, e $\mathcal{E}_{1,2}(\mathbf{r})=\rho_{1,2} \Lambda(\rho)$ está na direção do plano $(1,2)$. Nesse caso, novamente o operador de projeção é a identidade (pois $\boldsymbol{\nabla}(\boldsymbol{\nabla} \cdot \boldsymbol{\rho} \Lambda(\rho))=\nabla^{2} \boldsymbol{\rho} \Lambda(\rho)$ ). Então, ao invés de (7.4) a relação

$$
E_{1,2}(\rho)=E_{1,2}^{\operatorname{lin}}(\rho)\left(1-\frac{2 \alpha}{45 \pi}\left(\frac{e\left|\mathbf{E}^{\operatorname{lin}}(\rho)\right|}{m^{2}}\right)^{2}\right), \quad E_{3}=0 .
$$

deverá ser estabelecida. Se, além disso, a densidade de carga volumétrica $\kappa$ é independente também do raio $\rho, \kappa=$ const, o campo eletrico linear do fio infinito homogeneamente carregado, com raio $R$ e seu eixo coincidindo com o eixo 3

$$
\begin{gathered}
\mathbf{E}^{\operatorname{lin}}(\rho)=\Theta(R-\rho) \mathbf{E}^{<}(\rho)+\Theta(\rho-R) \mathbf{E}^{>}(\rho), \\
\mathbf{E}^{<}(\rho)=\frac{\kappa \rho}{2} \mathbf{e}_{\rho}, \mathbf{E}^{>}(\rho)=\frac{\kappa R^{2}}{2 \rho} \mathbf{e}_{\rho} .
\end{gathered}
$$

deve ser substituido em (7.10).

A mesma simetria do fio infinito acima é aplicável a uma espira circular carregando uma corrente constante com uma densidade invariante-O(2). Porém, agora o campo (6.15) tem a forma $\mathfrak{h}(\mathbf{r})=$ $\boldsymbol{e}_{3} \times \mathbf{r} \Lambda\left(\left|\boldsymbol{e}_{3} \times \mathbf{r}\right|\right)$. Agora $\boldsymbol{\nabla} \cdot \mathfrak{h}(\mathbf{r})=0$, e o operador de projeção em (6.17) é a identidade novamente, ou seja, $B_{i}^{\text {nl }}(\mathbf{r})=\mathfrak{h}_{i}(\mathbf{r})$.

Agora se tomamos uma corrente uniforme de densidade $\mathbf{j}$ por unidade de área, fluindo na direção positiva, e calcularmos o campo magnético linear produzido por ela, obtemos a relação linear

$$
\begin{gathered}
\mathbf{B}^{\operatorname{lin}}(\rho)=\Theta(R-\rho) \mathbf{B}^{<}(\rho)+\Theta(\rho-R) \mathbf{B}^{>}(\rho), \\
\mathbf{B}^{<}(\rho)=\frac{j \rho}{2} \mathbf{e}_{\phi}, \mathbf{B}^{>}(\rho)=\frac{j R^{2}}{2 \rho} \mathbf{e}_{\phi}
\end{gathered}
$$

a ser usada na expressão

$$
B_{\phi}(\rho)=B_{\phi}^{\operatorname{lin}}(\rho)\left(1-\frac{2 \alpha}{45 \pi}\left(\frac{e B_{\phi}^{\operatorname{lin}}(\rho)}{m^{2}}\right)^{2}\right)
$$

que segue diretamente de (6.17) e (6.15) para o presente tipo de simetria do problema.

\footnotetext{
${ }^{4} \operatorname{sgn}(x)=\left\{\begin{array}{c}0, \text { se } x=0 \\ \frac{x}{|x|}, \text { se } x \neq 0\end{array}\right.$ é a função sinal.

${ }^{5} \mathrm{~A}$ coordenada radial do sistema de coordenadas cilíndricas é representada por $\rho$, e o versor nessa direção é dado por $\mathbf{e}_{\rho}=\frac{\rho}{\rho}$. O versor azimutal é denotado por $\mathbf{e}_{\phi}=\frac{\mathbf{e}_{3} \times \boldsymbol{\rho}}{\rho}$.
} 


\subsection{Correções para dipolos eletrostáticos e magnetostáticos}

Nesta seção, damos continuidade aos outros casos de simetria cilíndrica, mas agora sem quaisquer invariâncias de translação. Calculamos o campo não linear produzidos por distribuições de correntes estacionárias e não livres, como, por exemplo, dipolos magnéticos e elétricos, e usamos tais distribuições como modelo para exibirmos aplicações, como o cálculo do campo magnético produzido por pulsares, e uma justificativa para explicar o momento magnético anômalo de alguns bárions, como o $\Xi^{* 0}, \Delta^{0}$ e $\Sigma^{* 0}$, devido ao efeito de não linearidade, e comparar ocm alguns resultados já conhecidos.

\subsubsection{Simetria cilíndrica: A esfera uniformemente magnetizada}

Considere uma esfera de raio $R$ com magnetização uniforme $\mathbf{M}(\mathbf{r})=M \Theta(R-r) \mathbf{e}_{3}$ em repouso $^{6}$, na origem, e calculemos o campo magnético em todo espaço. Em última análise, substituimos a lagrangeana efetiva pela lagrangeana de Euler-Heisenberg, e tomamos o limite de grandes distâncias.

É bem conhecido o campo magnético devido a este dipolo em todo espaço ${ }^{7}$ [17], e é dado, em coordenadas esféricas, por

$$
\begin{aligned}
& \mathbf{B}_{\text {lin }}(\mathbf{r})=\left(\frac{2}{3} M \cos \theta \mathbf{e}_{r}-\frac{2}{3} M \sin \theta \mathbf{e}_{\theta}\right) \Theta(R-r) \\
& +\left(\frac{2}{3} \frac{R^{3}}{r^{3}} M \cos \theta \mathbf{e}_{r}+\frac{1}{3} \frac{R^{3}}{r^{3}} M \sin \theta \mathbf{e}_{\theta}\right) \Theta(r-R) .
\end{aligned}
$$

Calculemos (6.15). A magnetização é dada por

$$
\begin{aligned}
& \frac{-54}{\mathfrak{L}_{\mathfrak{F} \mathfrak{F}} M^{3}} \mathbf{M}_{\mathrm{nl}}(\mathbf{r})=\frac{27}{M^{3}} \mathbf{B}_{\text {lin }}(\mathbf{r}) B_{\operatorname{lin}}^{2}(\mathbf{r})=\left(8 \cos \theta \mathbf{e}_{r}-8 \sin \theta \mathbf{e}_{\theta}\right) \Theta(R-r) \\
& +\left[\left(2 \frac{R^{9}}{r^{9}} \cos \theta+6 \frac{R^{9}}{r^{9}} \cos ^{3} \theta\right) \mathbf{e}_{r}+\left(4 \frac{R^{9}}{r^{9}} \sin \theta-3 \frac{R^{9}}{r^{9}} \sin ^{3} \theta\right) \mathbf{e}_{\theta}\right] \Theta(r-R) .
\end{aligned}
$$

E sua divergência, em termos dos harmônicos esféricos ${ }^{8} Y_{1}^{0}(\Omega)=\frac{1}{2} \sqrt{\frac{3}{\pi}} \cos \theta$

e $Y_{3}^{0}(\Omega)=\frac{1}{4} \sqrt{\frac{7}{\pi}}\left(5 \cos ^{3} \theta-3 \cos \theta\right)$, por

$$
\begin{aligned}
& \frac{-54}{\mathfrak{L}_{\mathfrak{F} \mathfrak{F}} M^{3}} \boldsymbol{\nabla} \cdot \mathbf{M}_{\mathrm{nl}}(\mathbf{r})=-16 \delta(r-R) \sqrt{\frac{\pi}{3}} Y_{1}^{0}(\Omega) \\
& +\frac{R^{9}}{r^{9}} \delta(r-R)\left(\frac{56}{5} \sqrt{\frac{\pi}{3}} Y_{1}^{0}(\Omega)+\frac{24}{5} \sqrt{\frac{\pi}{7}} Y_{3}^{0}(\Omega)\right) \\
& +\frac{R^{9}}{r^{10}} \Theta(r-R)\left(-72 \sqrt{\frac{\pi}{3}} Y_{1}^{0}(\Omega)-24 \sqrt{\frac{\pi}{7}} Y_{3}^{0}(\Omega)\right) .
\end{aligned}
$$

${ }^{6} \Theta(x)=\left\{\begin{array}{c}0, \text { se } x<0, \\ 1 / 2, \text { se } x=0, \text { é a função de Heaviside. } \\ 1, \text { se } x>0 .\end{array}\right.$

${ }^{7}$ Esta distribuição de dipolo é bem representada por uma corrente de polarização singular na superfície, girando em sentido anti-horário em relação ao eixo $x_{3}$. Ou seja, esta corrente pode ser representada por $\mathbf{j}(\mathbf{r})=3 \frac{\mathcal{M} \times \mathbf{r}}{r^{4}} \delta(r-R)$ onde $\mathcal{M}=\frac{\mathbf{M}}{4 \pi R^{3}}$ é o momento de dipolo magnético total associado a esta esfera.

${ }^{8}$ No sistema de coordenadas esféricas, os harmônicos esféricos são denotados por $Y_{l}^{m}(\Omega)=Y_{l}^{m}(\theta, \varphi)$, onde $\Omega$ é o ângulo sólido representado pelo ângulo zenital $\theta$ e o ângulo azimutal $\varphi, m$ é o número inteiro azimutal, e $l$ é o número inteiro zenital. 
Separamos variáveis nas partes radiais e angulares (esféricos harmônicos normalizados)

$$
\frac{-54}{\mathfrak{L}_{\mathfrak{F F}} M^{3}} \nabla \cdot \mathbf{M}_{\mathrm{nl}}(\mathbf{r})=\sum_{j} R_{j}(r) Y_{j}^{0}(\Omega),
$$

onde, usando a relação de ortogonalidade em $\int Y_{j}^{0}(\Omega) Y_{l}^{* m}(\Omega) d \Omega=\delta_{j l} \delta_{0 m}$.

$$
R_{j}(r)=\frac{-54}{\mathfrak{L}_{\mathfrak{F F}} M^{3}} \int \nabla \cdot \mathbf{M}_{\mathrm{nl}}(\mathbf{r}) Y_{j}^{0}(\Omega) \mathrm{d} \Omega .
$$

Para a corrente calculada em (7.18), obtemos

$$
\begin{aligned}
& R_{j}(r)=\left[\left(-16+\frac{56}{5} \frac{R^{9}}{r^{9}}\right) \delta(r-R)-72 \frac{R^{9}}{r^{10}} \Theta(r-R)\right] \sqrt{\frac{\pi}{3}} \delta_{1 j} \\
& +\left[\frac{24}{5} \frac{R^{9}}{r^{9}} \delta(r-R)-24 \frac{R^{9}}{r^{10}} \Theta(r-R)\right] \sqrt{\frac{\pi}{7}} \delta_{3 j} .
\end{aligned}
$$

Escrevemos a função de Green da seguinte forma [18]:

$$
\frac{1}{\left|\mathbf{r}-\mathbf{r}^{\prime}\right|}=\sum_{l=0}^{\infty} \sum_{m=-l}^{l} \frac{4 \pi}{2 l+1} Y_{l}^{m}(\Omega) Y_{l}^{* m}\left(\Omega^{\prime}\right)\left\{\begin{array}{ll}
\frac{r^{\prime l}}{r^{l+1}}, & \text { se } r>r^{\prime} \\
\frac{r^{l}}{r^{\prime l+1}}, & \text { se } r<r^{\prime}
\end{array} .\right.
$$

Calculemos (6.17), usando (6.15), (7.19), (7.21) e (7.22). Separamos as integrais em duas regiões do espaço, e, por ortogonalidade dos esféricos harmônicos, a integral sobre o ângulo sólido seleciona apenas os termos tais que $m=0$ (simetria azimutal) e $l=j$ :

$$
\begin{aligned}
& \frac{-54}{\mathfrak{L}_{\mathfrak{F F}} M^{3}} \mathbf{B}_{\mathrm{nl}}(\mathbf{r})=\frac{-54}{\mathfrak{L}_{\mathfrak{F} \mathfrak{F}} M^{3}} \mathbf{M}_{\mathrm{nl}}(\mathbf{r}) \\
& +\sum_{j} \frac{1}{2 j+1} \boldsymbol{\nabla}\left(\int_{0}^{r} R_{j}\left(r^{\prime}\right) \frac{r^{\prime j+2}}{r^{j+1}} \mathrm{~d} r^{\prime}+\int_{r}^{\infty} R_{j}\left(r^{\prime}\right) \frac{r^{j}}{r^{\prime j-1}} \mathrm{~d} r^{\prime}\right) Y_{j}^{0}(\Omega) .
\end{aligned}
$$

Agora atuamos o gradiente em coordenadas esféricas para obter

$$
\begin{aligned}
& \frac{-54}{\mathfrak{L}_{\mathfrak{F} \mathfrak{F}} M^{3}} \mathbf{B}_{\mathrm{nl}}(\mathbf{r})=\frac{-54}{\mathfrak{L}_{\mathfrak{F} \mathfrak{F}} M^{3}} \mathbf{M}_{\mathrm{nl}}(\mathbf{r}) \\
& +\sum_{j} \frac{1}{2 j+1}\left[\Sigma_{j}(r) Y_{j}^{0}(\Omega) \mathbf{e}_{r}+\Xi_{j}(r) \frac{\partial}{\partial \theta} Y_{j}^{0}(\Omega) \mathbf{e}_{\theta}\right],
\end{aligned}
$$

onde

$$
\begin{aligned}
& \Sigma_{j}(r)=-(j+1) \int_{0}^{r} R_{j}\left(r^{\prime}\right) \frac{r^{\prime j+2}}{r^{j+2}} \mathrm{~d} r^{\prime}+j \int_{r}^{\infty} R_{j}\left(r^{\prime}\right) \frac{r^{j-1}}{r^{\prime j-1}} \mathrm{~d} r^{\prime} \\
& \mathrm{e} \\
& \Xi_{j}(r)=\int_{0}^{r} R_{j}\left(r^{\prime}\right) \frac{r^{\prime j+2}}{r^{j+2}} \mathrm{~d} r^{\prime}+\int_{r}^{\infty} R_{j}\left(r^{\prime}\right) \frac{r^{j-1}}{r^{\prime j-1}} \mathrm{~d} r^{\prime}
\end{aligned}
$$


Portanto, por (7.21)

$$
\begin{aligned}
& \Sigma_{j}(r)=\sqrt{\frac{\pi}{3}}\left[-\frac{64}{5} \delta_{1 j}+\frac{432}{55} \frac{r^{2}}{R^{2}} \delta_{3 j}\right] \Theta(R-r) \\
& +\sqrt{\frac{\pi}{3}}\left[\left(\frac{168}{5} \frac{R^{3}}{r^{3}}-32 \frac{R^{9}}{r^{9}}\right) \delta_{1 j}+\left(\frac{24}{5} \frac{R^{5}}{r^{5}}-\frac{336}{11} \frac{R^{9}}{r^{9}}\right) \delta_{3 j}\right] \Theta(r-R), \\
& \mathrm{e} \\
& \Xi_{j}(r)=\sqrt{\frac{\pi}{7}}\left[-\frac{64}{5} \delta_{1 j}+\frac{144}{55} \frac{r^{2}}{R^{2}} \delta_{3 j}\right] \Theta(R-r) \\
& +\sqrt{\frac{\pi}{7}}\left[\left(-\frac{84}{5} \frac{R^{3}}{r^{3}}+4 \frac{R^{9}}{r^{9}}\right) \delta_{1 j}+\left(-\frac{6}{5} \frac{R^{5}}{r^{5}}+\frac{42}{11} \frac{R^{9}}{r^{9}}\right) \delta_{3 j}\right] \Theta(r-R) .
\end{aligned}
$$

Substituindo (7.17) e (7.25) em (7.23), encontramos o campo magnético em todo espaço:

$$
\begin{aligned}
& \frac{-54}{\mathfrak{L}_{\mathfrak{F} \mathfrak{F}} M^{3}} \mathbf{B}_{\mathrm{nl}}(\mathbf{r})= \\
& \left\{\left[\left(\frac{88}{15}-\frac{324}{385} \frac{r^{2}}{R^{2}}\right) \cos \theta+\frac{108}{77} \frac{r^{2}}{R^{2}} \cos ^{3} \theta\right] \mathbf{e}_{r}\right. \\
& \left.+\left[\left(-\frac{88}{15}-\frac{432}{385} \frac{r^{2}}{R^{2}}\right) \sin \theta+\frac{108}{77} \frac{r^{2}}{R^{2}} \sin ^{3} \theta\right] \mathbf{e}_{\theta}\right\} \Theta(R-r) \\
& +\left\{\left[\left(\frac{28}{5} \frac{R^{3}}{r^{3}}-\frac{18}{35} \frac{R^{5}}{r^{5}}-\frac{2}{33} \frac{R^{9}}{r^{9}}\right) \cos \theta+\left(\frac{6}{7} \frac{R^{5}}{r^{5}}+\frac{6}{11} \frac{R^{9}}{r^{9}}\right) \cos ^{3} \theta\right] \mathbf{e}_{r}\right. \\
& \left.+\left[\left(\frac{14}{5} \frac{R^{3}}{r^{3}}+\frac{18}{35} \frac{R^{5}}{r^{5}}+\frac{56}{33} \frac{R^{9}}{r^{9}}\right) \sin \theta+\left(-\frac{9}{14} \frac{R^{5}}{r^{5}}-\frac{21}{22} \frac{R^{9}}{r^{9}}\right) \sin ^{3} \theta\right] \mathbf{e}_{\theta}\right\} \Theta(r-R) .
\end{aligned}
$$

Para a região interna da esfera, i.e., quando $r<R$, observamos que essa solução é regular na origem, e respeita a simetria do problema, pois $\mathbf{B}_{\mathrm{nl}}(0)=-\frac{22}{405} \mathfrak{L}_{\mathfrak{F} \mathfrak{F}} M^{3} \mathbf{e}_{3}$, na direção axial, como esperado. Para a região externa da esfera, i.e., quando $r>R$, constatamos que o campo possui três potências da razão entre o raio da esfera $R$ e a distância da origem $r$ : uma à nona potência, outra à quinta potência e outra, o caso que mais nos interessa, à terceira potência, bem como a ordem da potência linear de dipolo. Isto significa que, a grandes distâncias da origem, o campo não linear cai da mesma forma que o campo linear, diferentemente do caso do campo elétrico devido ao monopolo, cuja parte não linear cai na sexta potência, enquanto a parte linear cai à segunda potência (7.4). Logo, impondo $r>>R$,

$$
\left.\mathbf{B}_{\mathrm{nl}}(\mathbf{r})\right|_{r>>R}=-\frac{7}{135} \mathfrak{L}_{\mathfrak{F} \mathfrak{F}} \frac{M^{3} R^{3}}{r^{3}}\left(2 \cos \theta \mathbf{e}_{r}+\sin \theta \mathbf{e}_{\theta}\right) .
$$

Em termos do campo linear $\left.\mathbf{B}_{\text {lin }}(\mathbf{r})\right|_{r>R}=\frac{1}{3} \frac{M R^{3}}{r^{3}}\left(2 \cos \theta \mathbf{e}_{r}+\sin \theta \mathbf{e}_{\theta}\right)$, o campo corrigido (6.5) é a soma do campo linear com o campo não linear, i.e.,

$$
\left.\mathbf{B}(\mathbf{r})\right|_{r>>R}=\mathbf{B}_{\text {lin }}(\mathbf{r})\left(1-\frac{7}{135} \mathfrak{L}_{\mathfrak{F} \mathfrak{F}} M^{2}\right) .
$$

Para a lagrangeana de Euler-Heisenberg, $\mathfrak{L}_{\mathfrak{F F}}=\frac{4 \alpha e^{2}}{45 \pi m^{4}}$, o campo é dado por

$$
\left.\mathbf{B}(\mathbf{r})\right|_{r>>R}=\mathbf{B}_{\operatorname{lin}}(\mathbf{r})\left(1-\frac{28}{135} \frac{\alpha}{45 \pi}\left(\frac{e M}{m^{2}}\right)^{2}\right) \text {. }
$$




\subsubsection{Campo magnético produzido por pulsares de Rádio, Raios-X, e Magnetars}

Com a equação (7.26) em mãos, podemos modelar a produção de campo magnético em corpos fortemente magnetizados, como os pulsares de Rádio, de Raios-X e de magnetars. Se o campo magnético na superfície de um pulsar é próximo do valor crítico de Schwinger $4,4 \cdot 10^{13} G$, mas ainda menor, a correção (7.26), próxima à superfície do polo norte magnético de um pulsar é em torno de $1,6 \cdot 10^{9} G[19]$, i.e., $3,7 \cdot 10^{-5}$ do campo magnético linear (a não linearidade máxima). Alguns magnetars, como por exemplo SGR 0418+5729 [20], possui esta ordem de grandeza (em torno de 1/4 do valor crítico de Schwinger). A grandes distâncias do pulsar, esta razão converge para $3,2 \cdot 10^{-5}$ (a não linearidade mínima), i.e., a correção não sofre mudanças consideráveis devido à distância, porque o maior termo não linear cai da mesma forma que o termo linear. Portanto, suficientemente longe do pulsar, esta razão é constante, e efeitos não lineares podem ainda serem observados.

Prevemos que na superfície de uma estrela de nêutrons, próximo ao polo norte magnético, a não linearidade é máxima. Estimando a ordem de grandeza da razão entre $B^{\mathrm{nl}}$ e $B$ em termos da magnetização da estrela em Gauss, pulsares do tipo Raios-X, como 1E 1207.4-5209 (10 $\left.{ }^{11} G\right)$ [21], possuem correção na faixa de $10^{2}-10^{4} G$ na superfície. Pulsares de Rádio, como PSR J1119-6127 $\left(4,1 \cdot 10^{13} G\right)[22]$, possuem correção em torno de $10^{7} G$ na superfície, e a correção mais importante se aplica para pulsares e magnetars cujo campo na superfície vale em torno de $10^{13} G$, como SGR 0418+5729 mencionado anteriormente, cuja correção é em torno de $10^{8} G$ de seu campo magnético na superfície.

\subsubsection{Momento magnético anômalo dos Bárions $\Xi^{* 0}, \Delta^{0}$ e $\Sigma^{* 0}$}

Alguns bárions que possuem magnetização não tão intensa, comparada ao campo de Schwinger, podem ter seus momentos magnéticos anômalos explicados pelo efeito de não linearidade. Chamamos atenção aqui ao bárion excitado $\Xi^{* 0}$, cujo momento magnético é estimado [23] por $0,16(4)$, e o membro decupleto $\Delta^{0}$, com momento magnético -0,035(2).Então a correção linear à magnetização (assumindo que seu raio possui a mesma ordem de grandeza do neutron) é em torno de $0.23<<1$ para $\Xi^{* 0}$ e $0,01<<1$ para $\Delta^{0}$. A correção para $\Xi^{* 0}$ está dentro do alcance dos erros teóricos admitidos em [23], que evidencia que os cálculos dos momentos magnéticos se referem à estrutura de quarks e estão em congruência com a extrapolação ao valor observável da massa do píon em QCD (com auxílio da teoria de perturbação quiral). Da mesma forma para $\Delta^{0}$, a correção de 0,01 aproximadamente se enquadra no alcance admitido de $2 / 35=0,057$. Mas outros cálculos (aqueles em [24] realizados com o auxílio de teoria de perturbação quiral) fornecem -0,17(4) para o momento magnético de $\Delta^{0}$, e, correspondentemente, produzem uma correção não linear de 0,26 , enquanto que a incerteza teórica é de 0,23. Outro candidato tratável com nossas fórmulas pode ser $\Sigma^{* 0}$. Valores experimentais não existem para esses bárions, mas há predições teóricas dentro da mesma teoria que produz boa coincidência com momentos magnéticos experimentalmente medidos independente se os últimos estão disponíveis, inclusive nêutrons e prótons.

\subsubsection{Simetria cilíndrica: A esfera uniformemente polarizada}

O campo elétrico de um dipolo elétrico pode ser calculado de forma análoga, mutatis mutandis, ao caso anterior do dipolo magnético, embora usamos a projeção longitudinal (6.11) ao invés da projeção transversal. Portanto, consideremos uma esfera de raio $R$, com polarização $\mathbf{P}=P \Theta(R-r) \mathbf{e}_{3}$. O 
campo elétrico linear é bem conhecido ${ }^{9}[17]$ e é dado por

$$
\begin{aligned}
& \mathbf{E}_{\text {lin }}(\mathbf{r})=\left(-\frac{1}{3} P \cos \theta \mathbf{e}_{r}+\frac{1}{3} P \sin \theta \mathbf{e}_{\theta}\right) \Theta(R-r) \\
& +\left(\frac{2}{3} \frac{R^{3}}{r^{3}} P \cos \theta \mathbf{e}_{r}+\frac{1}{3} \frac{R^{3}}{r^{3}} P \sin \theta \mathbf{e}_{\theta}\right) \Theta(r-R) .
\end{aligned}
$$

Portanto, realizando os mesmos cálculos do caso abordado na sessão anterior, encontramos o campo elétrico:

$$
\begin{aligned}
& \frac{-54}{\mathfrak{L}_{\mathfrak{F} \mathfrak{F}} P^{3}} \mathbf{E}_{\mathrm{nl}}(\mathbf{r})= \\
& \left\{\left[\left(-\frac{13}{15}+\frac{324}{385} \frac{r^{2}}{R^{2}}\right) \cos \theta-\frac{108}{77} \frac{r^{2}}{R^{2}} \cos ^{3} \theta\right] \mathbf{e}_{r}\right. \\
& \left.+\left[\left(\frac{13}{15}+\frac{432}{385} \frac{r^{2}}{R^{2}}\right) \sin \theta-\frac{108}{77} \frac{r^{2}}{R^{2}} \sin ^{3} \theta\right] \mathbf{e}_{\theta}\right\} \Theta(R-r) \\
& +\left\{\left[\left(\frac{2}{5} \frac{R^{3}}{r^{3}}+\frac{18}{35} \frac{R^{5}}{r^{5}}+\frac{68}{33} \frac{R^{9}}{r^{9}}\right) \cos \theta+\left(-\frac{6}{7} \frac{R^{5}}{r^{5}}+\frac{60}{11} \frac{R^{9}}{r^{9}}\right) \cos ^{3} \theta\right] \mathbf{e}_{r}\right. \\
& \left.+\left[\left(\frac{1}{5} \frac{R^{3}}{r^{3}}-\frac{18}{35} \frac{R^{5}}{r^{5}}+\frac{76}{33} \frac{R^{9}}{r^{9}}\right) \sin \theta+\left(\frac{9}{14} \frac{R^{5}}{r^{5}}-\frac{45}{22} \frac{R^{9}}{r^{9}}\right) \sin ^{3} \theta\right] \mathbf{e}_{\theta}\right\} \Theta(r-R) .
\end{aligned}
$$

E, a grandes distâncias, (6.5) é dada por

$$
\left.\mathbf{E}(\mathbf{r})\right|_{r>>R}=\mathbf{E}_{\text {lin }}(\mathbf{r})\left(1-\frac{1}{270} \mathfrak{L}_{\mathfrak{F F}} P^{2}\right),
$$

onde $\left.\mathbf{E}_{\text {lin }}(\mathbf{r})\right|_{r>R}=\frac{1}{3} \frac{P R^{3}}{r^{3}}\left(2 \cos \theta \mathbf{e}_{r}+\sin \theta \mathbf{e}_{\theta}\right)$ é o campo elétrico linear externo.

Para a lagrangeana de Euler-Heisenberg, $\mathfrak{L}_{\mathfrak{F} \mathfrak{F}}=\frac{4 \alpha e^{2}}{45 \pi m^{4}}$, e o campo é dado por

$$
\left.\mathbf{E}(\mathbf{r})\right|_{r>>R}=\mathbf{E}_{\operatorname{lin}}(\mathbf{r})\left(1-\frac{2}{135} \frac{\alpha}{45 \pi}\left(\frac{e P}{m^{2}}\right)^{2}\right) \text {. }
$$

\footnotetext{
${ }^{9}$ Esta distribuição de dipolo é bem representada por uma densidade de carga de polarização singular na superfície. Ou seja, esta densidade de carga pode ser representada por $j_{0}^{\mathrm{nl}}(\mathbf{r})=3 \frac{\mathbf{p} \times \mathbf{r}}{r^{4}} \delta(r-R)$ onde $\mathbf{p}=\frac{\mathbf{P}}{4 \pi R^{3}}$ é o momento de dipolo elétrico total associado a esta esfera.
} 


\section{Capítulo 8}

\section{Modelos estacionários de solução exata e a auto-energia do elétron}

Um problema intrínseco da teoria linear de Maxwell certamente reside na inconsistência do cálculo da auto-energia de cargas puntiformes. Um modelo não perturbativo muito utilizado, em que essa inconsistência é eliminada, é o modelo de Born-Infeld [31], onde, em analogia à partícula puntiforme relativística, limitada pela velocidade da luz c, há um campo de magnitude limitada devido à dependência de uma raiz quadrada na lagrangeana do invariante relativístico $\mathfrak{F}$. Na eletrodinâmica de Born-Infeld, a energia total do campo eletromagnético é finita e o campo elétrico é regular em todos os lugares. Essa teoria apresenta propriedades interessantes relativas à propagação de ondas, tais como a ausência de ondas choques e birrefringência. Na QED, da lagrangeana de Euler-Heisenberg podemos considerar somente o espalhamento de dois fótons, implicando na seleção do termo quadrático em $\mathfrak{F}$. Nesse capítulo mostramos que tal teoria, o modelo quártico, introduz um mínimo de não linearidade que é suficiente para sanar o problema da convergência da autoenergia da carga puntiforme. Generalizando a mais número de loops, na QED, selecionamos termos polinomiais de potências arbitrárias. Chamamos esse modelo de modelo polinomial. Se truncamos (como em [1]) a série de Taylor expandida a quaisquer potências do campo invariante $\mathfrak{F}$, a solução das correspondentes equações de Maxwell para o campo eletrostático de uma carga puntiforme configura um campo de energia sempre finita. Isto significa que a ação efetiva da QED, definida como a transformação de Legendre das funções de Green do fóton [45], é tomada na aproximação local, ou infravermelho $[32,1,46]$. Isso pode ser pensado como a ação de Euler-Heisenberg calculada com a acurácia de quaisquer números de loops. Mostramos, nessa seção, que tais modelos removem a singularidade que origina divergência no cálculo da auto-energia do elétron. O foco principal está já no modelo minimalmente não-linear, o modelo quártico, que é suficiente para a convergência da integral da energia em todo o espaço.

\subsection{O modelo de Born-Infeld}

O modelo de Born-Infeld é um exemplo particular de eletrodinâmica não linear, e tem o nome de físicos Max Born e Leopold Infeld quem primeiro propôs. Foi historicamente introduzida na década de 30 para remover a divergência de auto-energia do elétron em eletrodinâmica clássica, introduzindo um limite superior do campo elétrico na origem. 
A lagrangeana de Born-Infeld é dada por

$$
L=a^{2}\left(1-\sqrt{1+\frac{2 \mathfrak{F}}{a^{2}}-\frac{\mathfrak{G}^{2}}{a^{4}}}\right) .
$$

O coeficiente devido ao termo quártico em sua expansão em séries de potências é

$$
\left.L_{\mathfrak{F} \mathfrak{F}}\right|_{\mathfrak{F}=\mathfrak{G}=\mathfrak{0}}=a^{-2} .
$$

Calculando este termo $\left.L_{\mathfrak{F} \mathfrak{F}}\right|_{\mathfrak{F}=\mathfrak{G}=\mathfrak{o}}$ diretamente da QED

$$
\left.L_{\mathfrak{F} \mathfrak{F}}\right|_{\mathfrak{F}=\mathfrak{G}=\mathfrak{o}} \equiv \gamma=\frac{e^{4}}{45 \pi^{2} m^{4}},
$$

impomos que o parâmetro livre em (8.1) seja

$$
a=m^{2} \frac{3 \sqrt{ } 5}{4 \alpha}=230 m^{2}=5,7 \times 10^{7} \mathrm{eV}^{2}=E_{\mathrm{Sch}}^{\mathrm{HL}} \frac{3 \pi \sqrt{ } 5}{e^{\mathrm{HL}}} \simeq 70 E_{\mathrm{Sch}}^{\mathrm{HL}}=26 \cdot 10^{16} \frac{\mathrm{V}}{\mathrm{cm}}
$$

A equação de movimento não linear, onde há somente um campo invariante, é

$$
\partial_{\mu} \frac{F^{\mu \nu}(x)}{\sqrt{1+\frac{2 \mathfrak{F}(x)}{a^{2}}}}=j^{\nu}(x) .
$$

Tomando $j^{0}(x)=e \delta^{3}(\mathbf{r}), \mathbf{j}(x)=\mathbf{0}$, similarmente a nosso caso, a solução esfericamente simétrica para o campo eletrostático deve satisfazer a equação

$$
\frac{E}{\sqrt{1-\frac{E^{2}}{a^{2}}}}=\frac{e}{4 \pi r^{2}} \text {. }
$$

Ou seja,

$$
E(r)=\frac{e}{4 \pi r^{2}}\left(1+\left(\frac{e}{4 \pi a r^{2}}\right)^{2}\right)^{-\frac{1}{2}}, \quad E(0)=a .
$$

Dessa forma, quando $r=0$, este campo é constante, e a singularidade é completamente removida, e essa é a grande vantagem desse modelo, que por essa razão tanto tem se popularizado.

\subsection{O modelo quártico}

No modelo de Born-Infeld, vimos que é necessário regularizar o campo elétrico de forma que seu valor na origem, onde há uma carga puntiforme singular, é finito (8.3). Com isso, o cálculo da energia contida no campo elétrico converge em todas as regiões do espaço. Nesta seção, abordaremos um modelo minimalmente não linear, e mostraremos que o mínimo de não linearidade, adicionando um termo quadrático em $\mathfrak{F}(x)$ na lagrangeana, já é suficiente para garantir a convergência da energia do campo. Definimos a a lagrangeana de uma teoria eletrodinâmica minimalmente não linear por

$$
L(x)=-\mathfrak{F}(x)+\frac{\gamma}{2}(\mathfrak{F}(x))^{2},
$$

onde a constante de acoplamento $\gamma$ é pequena o suficiente, e possui dimensão do inverso da quarta potência da massa. 
O princípio da causalidade e unitariedade aplicado à ação efetiva local de quailquer eletrodinâmica não linear resulta em alguns requisitos [32], entre as quais o primeiro é $\gamma>0$. Os outros requisitos (no presente caso, onde o segundo campo invariante $\mathfrak{G}=\mathbf{E} \cdot \mathbf{B}$ não está envolvido) se reduzem a $\frac{\mathrm{d} L}{\mathrm{~d} \mathfrak{F}} \leqslant 0$, e $\frac{\mathrm{d} L}{\mathrm{~d} \mathfrak{F}}+\frac{\mathrm{d}^{2} L}{\mathrm{~d} \mathfrak{F}^{2}} \leqslant 0$. Estas condições são satisfeitas pelo campo sempre, desde que $\mathfrak{F}<0$. Esta é uma vantagem, em comparação ao modelo de Born-Infeld [31], que se torna inconsistente a valores muito altos do campo elétrico. Aqui trataremos o caso do campo elétrico isoladamente em um referencial de Lorentz especial, $2 \mathfrak{F}(x)=-E^{2}$, nessa dissertação.

Devemos observar que o modelo (8.4) possui a propriedade desejável de finitude para o energia do campo de uma carga pontual, como o modelo de the Born-Infeld [31], diferentemente do que ocorre com outras teorias lineares, como a do eletromagnetismo e sua extensão quantizada, a QED. Se pensarmos nessa teoria para posterior quantização, estaremos de certa forma fixando a constante de acoplamento $\gamma$. O termo quártico $\mathfrak{F}^{2}$, adicinado à lagrangeana, participaria do espalhamento fóton por fóton, como por exemplo o espalhamento Delbrück, o centro de Coulomb, formação de um meio anisotrópico para a propagação do fóton em um campo externo, modificação da lei de Coulomb em um campo magnético [40], renormalização dos momentos elétricos e magnéticos no vácuo [1] ${ }^{1}$. Além desses fenômenos não lineares, somente aqueles associados com átomos tem sido recentemente observados [41]. Entretanto, a precisão experimental nesses últimos casos não é suficiente para determinar a constante $\gamma$ em (8.4). Por outro lado, o mesmo termo quártico contribui para os momentos magnéticos anômalos do elétron e do múon, medidos e calculados na QED com grande precisão, e para o espectro atômico. Recentemente um novo processo dependendo do espalhamento de fóton por fóton foi proposto para ser medido no colisor de átomos pesados no LHC [42]. Mas mesmo nesses casos experimentalmente calculáveis, uma separação direta da contribuição de $\gamma$ poderia ser difícil, e, portanto, desnecessária. É suficiente se referir à QED como a teoria perfeitamente responsável por estes dados, relacionados ao eletromagnetismo, com exceção, talvez à discrepância com o momento magnético do múon. Correspondentemente, a escala da constante de acoplamento $\gamma$ serpa extraída da QED, mais precisamente, será tomada do funcional da ação efetiva de Euler-Heisenberg [2] que contém a amplitude de espalhamento fóton por fóton no primeiro termo da expansão de Taylor do invariante do campo ${ }^{2}$.

\subsubsection{Equações para os campos}

Dentro do modelo quártico, podemos resolver a equação (3.4) de forma exata. O princípio da mínima ação aplicado ao funcional $S[A]=\int L(x) d^{4} x$ fornece equações de movimento $\frac{\delta S}{\delta A_{\nu}(x)}=0$, que são o segundo par de equações de Maxwell, que são não lineares:

$$
\partial_{\mu}\left[(1-\gamma \mathfrak{F}(x)) F_{0}^{\mu}\right]=j_{0}(x) .
$$

Estamos interessado na solução esfericamente simétrica puramente eletrostática produzida por uma carga puntiforme e em repouso, colocada na origem, i.e., dada uma fonte $j_{0}(x)=e \delta^{3}(\mathbf{r})$. Portanto, estas equações são reduzidas a

$$
\begin{gathered}
\boldsymbol{\nabla} \cdot\left[\left(1+\frac{\gamma}{2} E^{2}(x)\right) \mathbf{E}(x)\right]=j_{0}(x), \\
\boldsymbol{\nabla} \times \mathbf{E}(x)=\mathbf{0}
\end{gathered}
$$

\footnotetext{
${ }^{1}$ Certamente, outra adição quártica, $\mathfrak{G}^{2}$, contribuiria a esses processos na mesma ordem, mas isto não é importante para nosso presente propósito.

${ }^{2}$ Cf. Ref.[44], onde os autores estão baseando suas estimativas do parâmetro livre na Lagrangeana de Born-Infeld em comparação com a QED.
} 
como calculado em (6.7). Definindo $\mathbf{E}_{\operatorname{lin}}(x)=\nabla a_{\operatorname{lin}}^{0}(x)$, onde $a_{\operatorname{lin}}^{0}(x)=\int d^{4} y D^{0 \rho}(x-y) j_{0}(y)$, a solução é análoga à apresentada na seção 5.4. Impondo independência temporal, obtemos:

$$
\left(1+\frac{\gamma}{2} E^{2}(r)\right) \mathbf{E}(\mathbf{r})=\mathbf{E}_{\text {lin }}(\mathbf{r}),
$$

que satisfaz as equações de Maxwell (8.6). Neste caso, o campo linear é dado pela Lei de Coulomb padrão:

$$
\mathbf{E}_{\text {lin }}(\mathbf{r})=\frac{e}{4 \pi r^{2}} \frac{\mathbf{r}}{r}
$$

\subsubsection{Uma solução exata para o modelo quártico}

Agora a equação (8.7) se torna cúbica em termos de $E(r)$

$$
\left(1+\frac{\gamma}{2} E^{2}(r)\right) E(r)=\frac{e}{4 \pi r^{2}}
$$

que fornece a solução dada pela fórmula de Tartaglia-Cardano [43] (Apêndice 5):

$$
E(r)=\sqrt[3]{\frac{E_{\operatorname{lin}}(r)}{\gamma}+\sqrt{\left(\frac{E_{\operatorname{lin}}(r)}{\gamma}\right)^{2}+\left(\frac{2}{3 \gamma}\right)^{3}}}+\sqrt[3]{\frac{E_{\operatorname{lin}}(r)}{\gamma}-\sqrt{\left(\frac{E_{\text {lin }}(r)}{\gamma}\right)^{2}+\left(\frac{2}{3 \gamma}\right)^{3}}},
$$

$\operatorname{com} E_{\text {lin }}(r)=\left|\mathbf{E}_{\text {lin }}(\mathbf{r})\right|=\frac{e}{4 \pi r^{2}}$.

Convém salientar que nessa equação cúbica há de fato uma única solução real, e outras duas complexas. Para mostrar isso, é suficiente notar que o discriminante quadrático é sempre positivo.

\subsubsection{Soluções assintóticas}

Estudamos os limites especiais quando o campo é pequeno o suficiente, isto é, a grandes distâncias da carga, quando esperamos que, em uma primeira aproximação, recuperamos o campo de Coulomb. Mais que isso, esperamos que o próximo perturbativo da expansão em séries de potências reproduza a solução de Lifschitz-Beretetsky-Pitayevsky (7.4). Com a solução geral em mãos, podemos deduzir inferir também o comportamento a curtas distâncias, onde o campo é forte.

\subsubsection{Solução perturbativa}

Primeiramente vamos encontrar a solução perturbativa para campos a grandes distâncias. Primeiro, notemos que $E$ é uma função ímpar em $E_{\text {lin }}$, i.e.:

$$
E\left(-E_{\text {lin }}\right)=-E\left(E_{\text {lin }}\right)
$$

Seja $f$ uma função tal que:

$$
f(x)=\sqrt[3]{a x+\sqrt{(a x)^{2}+b^{3}}}+\sqrt[3]{a x-\sqrt{(a x)^{2}+b^{3}}} .
$$

Sua primeira derivada é:

$$
f^{\prime}(x)=\frac{a}{3 \sqrt{a^{2} x^{2}+b^{3}}}\left(\sqrt[3]{\sqrt{a^{2} x^{2}+b^{3}}+a x}-\sqrt[3]{a x-\sqrt{a^{2} x^{2}+b^{3}}}\right),
$$


E sua terceira derivada é:

$$
\begin{aligned}
f^{\prime \prime \prime}(x) & =-\frac{a^{3}}{27\left(a^{2} x^{2}+b^{3}\right)^{\frac{5}{2}}}\left[\sqrt[3]{a x+\sqrt{a^{2} x^{2}+b^{3}}}\left(8 b^{3}-19 a^{2} x^{2}+9 a x \sqrt{a^{2} x^{2}+b^{3}}\right)\right. \\
& \left.+\sqrt[3]{a x-\sqrt{a^{2} x^{2}+b^{3}}}\left(-8 b^{3}+19 a^{2} x^{2}+9 a x \sqrt{a^{2} x^{2}+b^{3}}\right)\right] .
\end{aligned}
$$

Calculando essas funções no ponto $x=0$ :

$$
\begin{gathered}
f^{\prime}(0)=\frac{2 a}{3 b}, \\
f^{\prime \prime \prime}(0)=-\frac{16 a^{3}}{27 b^{4}} .
\end{gathered}
$$

Então expandimos $f(x)$ em séries de Taylor (somente potências ímpares, pois $f$ é uma função ímpar):

$$
f(x)=f^{\prime}(0) x+\frac{f^{\prime \prime \prime}(0)}{3 !} x^{3}+\mathcal{O}\left(x^{5}\right) .
$$

Portanto, por (8.15) e (8.16),

$$
f(x)=\frac{2 a}{3 b} x-\frac{(2 a)^{3}}{(3 b)^{4}} x^{3}+\mathcal{O}\left(x^{5}\right) .
$$

Comparando (8.12) a (D.13) identificamos $f(x)=E\left(E_{\mathrm{lin}}\right), x=E_{\mathrm{lin}}, a=\frac{1}{\gamma}$ e $b=\frac{2}{3} \frac{1}{\gamma}$ :

$$
E=\frac{2\left(\frac{1}{\gamma}\right)}{3\left(\frac{2}{3} \frac{1}{\gamma}\right)} E_{\text {lin }}-\frac{\left[2\left(\frac{1}{\gamma}\right)\right]^{3}}{\left[3\left(\frac{2}{3} \frac{1}{\gamma}\right)\right]^{4}} E_{\operatorname{lin}}^{3}+\mathcal{O}\left(E_{\operatorname{lin}}^{5}\right)
$$

Portanto, obtemos a solução perturbativa em terceira ordem:

$$
E=E_{\text {lin }}-\frac{\gamma}{2} E_{\text {lin }}^{3}+\mathcal{O}\left(E_{\text {lin }}^{5}\right)
$$

E obtemos a esperada solução de Lifschitz-Beretetsky-Pitayevsky no limite de campos pequenos, quando a lagrangeana do problema se reduz à de Euler-Heisenberg, $\gamma=\mathfrak{L}_{\mathfrak{F} \mathfrak{F}}^{\mathrm{EH}}=\left.\frac{\mathrm{d}^{2} \mathfrak{L}^{\mathrm{EH}}(\mathfrak{F}, 0)}{\mathrm{d} \mathfrak{F}^{2}}\right|_{\mathfrak{F}=0}=$ $\frac{e^{4}}{45 \pi^{2} m^{4}}$, cujo primeiro termo já representa o campo de Coulomb:

$$
E(r)=E_{\text {lin }}(r)\left(1-\frac{\gamma}{2} E_{\text {lin }}^{2}(r)\right), \text { para } r \rightarrow \infty .
$$

\subsubsection{Solução para campos fortes}

O outro caso de interesse é quando $E_{\text {lin }}>>\sqrt{\frac{8}{27 \gamma}}$. Nesse caso, podemos desprezar o segundo termo no discriminante, logo:

$$
E^{+} \simeq \sqrt[3]{\frac{2}{\gamma} E_{\text {lin }}}
$$

e

$$
E^{-} \simeq 0
$$


Para distâncias curtas, $r \rightarrow 0$, o comportamento assintótico da solução (8.10) é:

$$
E(r) \sim\left(\frac{2 E_{\operatorname{lin}}(r)}{\gamma}\right)^{\frac{1}{3}}=\left(\frac{e}{2 \pi \gamma}\right)^{\frac{1}{3}}\left(\frac{1}{r}\right)^{\frac{2}{3}}, \text { para } r \rightarrow 0
$$

que pode inclusive ser imediatamente obtido diretamente de (8.9) se desprezamos a unidade no lado esquerdo do termo quadrático, muito maior que a unidade neste limite. A teoria quártica, regida pelas equações não lineares (8.5), difere da teoria clássica eletrostática de Maxwell a medida que nos aproximamos da origem, onde se encontra uma carga elétrica singular em repouso. Na eletrostática de Maxwell, o campo é coulombiano e, portanto, proporcional ao inverso do quadrado da distância, i.e., caindo com $r^{-2}$ a medida que nos aproximamos da origem, enquanto na eletrostática da teoria quártica o campo elétrico cai de uma forma um pouco mais lenta, com $r^{-2 / 3}$. Devemos ressaltar que a supressão da singularidade é suficiente para providenciar a convergência das integrais que fornecem a energia da configuração do campo (8.10).

Convém notar também que o potencial escalar vai a zero quando nos aproximamos da carga, i.e., $a_{0}(r \rightarrow 0) \sim r^{\frac{1}{3}} \rightarrow 0$, e, no infinito, quando estamos afastados o suficiente da carga, o potencial também vai a zero, pois decai como Coulomb $a_{0}(r \rightarrow \infty) \sim r^{-1} \rightarrow 0$. Isso nos assegura que estamos dentro do critério de convergência estabelecido em (3.4), i.e., que impõe que $a_{0}(x)$ seja finito em todas as regiões do espaço (inclusive na origem), necessário para que se possa expandir $\frac{\delta \Gamma}{\delta A^{\beta}(x)} \mathrm{em}$ séries de potências, conforme foi desenvolvido.

\subsubsection{Finitude da energia do campo produzido por uma carga pun- tiforme}

O tensor de energia-momento de Noether para a densidade de lagrangeana (8.4) é

$$
T^{\rho \nu}=(1-\gamma \mathfrak{F}(x)) F^{\mu \nu} \partial^{\rho} A_{\mu}-\eta^{\rho \nu} L(x) .
$$

Subtraindo a derivada total $\partial_{\mu}\left[\left(1-\gamma \mathfrak{F}(x) F^{\mu \nu}\right) A^{\rho}\right]$, igual a $\left[\left(1-\gamma \mathfrak{F}(x) F^{\mu \nu}\right) \partial_{\mu} A^{\rho}\right]$ devido às equações (8.5), o tensor energia-momento invariante de calibre e simétrico sobre a transposição $\rho \leftrightarrows \nu$

$$
\Theta^{\rho \nu}=(1-\gamma \mathfrak{F}(x)) F^{\mu \nu} F_{\mu}^{\rho}-\eta^{\rho \nu} L(x)
$$

é obtido. Quando há somente campo elétrico esfericamente simétrico, a densidade de energia é

$$
\Theta^{00}=\left(1+\frac{\gamma E^{2}}{2}\right) E^{2}-\frac{E^{2}}{2}\left(1+\frac{\gamma E^{2}}{4}\right)=\frac{E^{2}}{2}+\frac{3 \gamma E^{4}}{8} .
$$

Multiplicando a equação (8.9) por $E$ obtemos a relação $\frac{\gamma}{2} E^{4}(r)=E^{\operatorname{lin}}(r) E(r)-E^{2}(r)$. Levando em conta a densidade de energia, temos

$$
\Theta^{00}=-\frac{1}{4} E^{2}(r)+\frac{3}{4} E^{\operatorname{lin}}(r) E(r) .
$$

Portanto, para determinar toda a energia eletrostática $\int \Theta^{00} \mathrm{~d} \mathbf{r}$ armazenada na solução (8.10) temos que calcular duas integrais. A primeira é:

$$
\int E^{2}(r) \mathrm{d} \mathbf{r}=e^{\frac{3}{2}}\left(\frac{3}{2 \gamma(4 \pi)^{2}}\right)^{\frac{1}{4}} \frac{3}{2} I_{1}
$$


onde:

$$
I_{1}=\int_{0}^{\infty} x^{\frac{2}{3}}\left(\sqrt[3]{\sqrt{1+x^{4}}+1}-\sqrt[3]{\sqrt{1+x^{4}}-1}\right)^{2} \mathrm{~d} x=0,885
$$

A segunda é:

$$
\int E^{\operatorname{lin}}(r) E(r) \mathrm{d} \mathbf{r}=e \int_{0}^{\infty} E(r) \mathrm{d} r=e^{\frac{3}{2}}\left(\frac{3}{2 \gamma(4 \pi)^{2}}\right)^{\frac{1}{4}} I_{2}
$$

onde:

$$
I_{2}=\int_{0}^{\infty} x^{-\frac{2}{3}}\left(\sqrt[3]{\sqrt{1+x^{4}}+1}-\sqrt[3]{\sqrt{1+x^{4}}-1}\right) \mathrm{d} x=3,984 .
$$

Finalmente a energia é finita, e vale:

$$
\int \Theta^{00} \mathrm{~d} \mathbf{r}=e^{\frac{3}{2}}\left(\frac{3}{2 \gamma(4 \pi)^{2}}\right)^{\frac{1}{4}} \frac{1}{4}\left(3 I_{2}-\frac{3}{2} I_{1}\right)=2.656 e^{\frac{3}{2}}\left(\frac{3}{2 \gamma(4 \pi)^{2}}\right)^{\frac{1}{4}}<\infty .
$$

\subsubsection{Cálculo da massa do campo elétrico em QED}

Para estimar o resultado (8.27) podemos substituir o valor da constante de acoplamento $\gamma$ em (8.4) de acordo com o termo quártico correspondente a densidade lagrangeana de Euler-Heisenberg $\mathfrak{L}^{\mathrm{EH}}(\mathfrak{F}, \mathfrak{G})$ em um loop, i.e., (note que $\alpha=\frac{e^{2}}{4 \pi}=\frac{1}{137}$ no sistema de unidades de Heaviside-Lorentz) [2]

$$
\gamma=\left.\frac{\mathrm{d}^{2} \mathfrak{L}^{\mathrm{EH}}(\mathfrak{F}, 0)}{\mathrm{d} \mathfrak{F}^{2}}\right|_{\mathfrak{F}=0}=\frac{e^{4}}{45 \pi^{2} m^{4}}
$$

onde $m$ é a massa do elétron, e $e$ é a carga eletrônica, tendo em mente que a carga puntiforme previamente referida possui esse valor. Com essa substituição a energia do campo eletrostático (8.27) do elétron considerado como um monopolo puramente elétrico fornece o resultado:

$$
\int \Theta^{00} \mathrm{~d}^{3} x=7.15 \alpha^{\frac{1}{4}} m=2,09 m,
$$

particamente duas vezes maior que a massa eletrônica.

Esse resultado exibe uma limitação do modelo quártico, pois espera-se que a massa do campo elétrico seja menor que $m$, pois há outras interações além da eletromagnética ocorrendo, e que devem contribuir à massa do elétron, além de que aqui foi considerado somente o diagrama de espalhamento de dois fótons, isto é, outros diagramas devem contribuir para o cálculo dessa energia. Mas a importância desse resultado reside no fato de que mesmo com um modelo minimalmente não linear já obtemos uma energia finita, o que não ocorre na teoria de Maxwell.

\subsection{O modelo polinomial}

Podemos agora generalizar nossas afirmações acerca da finitude do da energia do campo de uma carga pontual a quaisquer teorias de eletrodinâmica não lineares, com a densidade de lagrangeana efetiva $\mathfrak{L}(\mathfrak{F})$ - ao invés da função quártica $\frac{\gamma}{2}(\mathfrak{F}(x))^{2}$ usada em $(8.4)$ - sendo uma função qualquer de $\mathfrak{F}$ que cresce como uma potência finita de seu argumento, digamos $\mathfrak{F}^{n+1}, n \geqslant 1$, quando $\mathfrak{F} \rightarrow \infty$. 
Desprezando novamente a unidade na equação ao aparecer no lugar de (8.9) obtemos, ao invés de (8.30) que a singularidade do campo elétrico próximo à origem é

$$
E(r) \sim\left(\frac{e n !(-1)^{n+1}}{2^{n} 4 \pi \mathfrak{L}^{(n+1)} r^{2}}\right)^{\frac{1}{2 n+1}}
$$

onde $\mathfrak{L}^{(n+1)}$ é a $(n+1)$-ésima derivada de $\mathfrak{L}$ tomada em $\mathfrak{F}=0$. Por outro lado, o termo proncipal de contribuição próxima a origem à densidade de energia do campo calculada na energia de Noether $\Theta^{00}$ será, ao invés de $E^{4}$ em $(8.26)$, proporcional a $E^{2 n+2}$. Apesar da potência ser maior, a integral da energia do campo $\int \Theta^{00} \mathrm{~d} \mathbf{r}$ com a substituição de (8.30) converge no limite inferior como $\int_{0} r^{-\frac{2}{2 n+1}} \mathrm{~d} r$, i.e., mais rapidamente que em (8.26). A convergência a longas distâncias é sempre estabelecida pela condição de contorno que impõe que o campo deva ser o coulombiano $E(r) \sim E^{\text {lin }}(r)$, quando toda a não linearidade na equação de movimento pode ser desconsiderada. 


\section{Capítulo 9}

\section{Conclusão}

Nesta dissertação, mostramos que os resultados da não linearidade são modelo-independentes e aplicáveis a qualquer eletrodinâmica não linear, onde invariâncias U(1), Lorentz, C, P, T são respeitadas, incluindo QED. Tomando a aproximação de limite infravermelho, podemos levar em conta sistemas físicos onde as correções quânticas propostas nessa dissertação são verificadas, como, por exemplo, o campo produzido por estrelas de quarks [25] e por pulsares [7] e [19]. Se este campo é suficientemente forte, mas ainda abaixo do valor crítico de Schwinger $e / m^{2}$, a não linearidade é causada pelo espalhamento fóton por fóton (i.e., expalhamento de vértices de quatro fótons). Muitos artigos recentes foram publicados nesse contexto, como por exemplo em [1], [6] e [46], onde foi abordado o efeito magneto-elétrico.

Dentre os resultados novos obtidos nessa dissertação, foi deduzida uma fórmula geral para a corrente não linearmente induzida (5.9), a partir do tensor de polarização de quarta ordem (5.5), responsável pela polarização do vácuo devido ao espalhamento de dois fótons, necessária para se solucionar as equações de Euler-Lagrange (3.11) de uma teoria eletrodinâmica que satisfaz a aproximação infra-vermelho no vácuo.

No âmbito da eletrostática, foi obtida uma generalização para a lei de Coulomb: uma forma analítica para o campo eletrostático gerado por um monopolo, que leva em conta efeitos não lineares (8.10), que são devidos somente ao espalhamentos de dois fótons (caracterizados, segundo o modelo quártico, pela constante de acoplamento $\gamma$ ). Essa expressão analítica permite o cálculo da autoenergia de partículas puntiformes em repouso no vácuo, no qual foi mostrado em (8.27) que já no modelo minimalmente não linear, o quártico, há convergência da energia eletrostática em todo espaço (o que não ocorre na teoria linear de Maxwell). No limite de grandes distâncias com relação a um monopolo, consideramos a constante de acoplamento da QED, fornecida pela lagrangeana de Euler-Heisenberg $\gamma=\frac{4 \alpha e^{2}}{45 \pi m^{4}}$. Nesse limite, o primeiro termo da expansão em séries de potências do campo é justamente o campo Coulombiano já conhecido da teoria de Maxwell linear, e o seguinte termo, o de terceira ordem no campo, é o termo de correção fornecido pela fórmula de LifshitzBeretetsky-Pitayevsky (7.4), satisfazendo-se assim o princípio da correspondência. Como possível aplicação experimental, esse efeito pode ser apreciável nas proximidades de estrelas de quarks, onde as correções não lineares ao campo elétrico gerado por esse monopolo cai com $r^{-6}$ na região externa à esfera, ou seja, muito mais rápido que o campo Coulombiano linear, e sua não-linearidade se torna apreciável quando o medimos próximo à superfície dessa estrela.

Além disso, foi pela primeira vez calculada, perturbativamente, a generalização da lei de BiotSavart para dipolos esféricos homogeneamente magnetizados, em repouso no vácuo (7.26), analogamente também para o dipolo elétrico (7.30), e foi mostrado que a grandes distâncias a correção não linear cai da mesma forma que o campo linear, ou seja, $\sim r^{-3}$, mostrando que o dipolo magnético 
(7.28) e o dipolo elétrico (7.32) possuem uma correção altamente relevante, o que permite inferir, como possibilidade de aplicação experimental, o campo magnético não linear de pulsares fortemente magnetizados, cujas correções são da ordem de $10^{-5}$ da solução linear para o campo de magnetars, $10^{-6}$ para pulsares do tipo Rádio, e $10^{-7}-10^{-9}$ para pulsares do tipo raios-X. Além disso, esse efeito de não linearidade também justifica o momento magnético anômalo de alguns bárions, como $\Xi^{* 0}, \Delta^{0}$ e $\Sigma^{* 0}$. Outras partículas elementares, cujos momentos magnéticos são tais que seus campos magnéticos são mais intensos que o valor crítico de Schwinger, como o nêutron, não podem ser tratados pelo modelo proposto nessa dissertação, pois são altos demais para que se possa tratar perturbativamente. Com isso, não podemos tratar o momento magnético anômalo do nêutron dessa forma, sendo necessário resolver a equação (5.9) de forma analítica, o que pode ser considerado em um trabalho futuro.

Quanto aos modelos de solução exata para o cálculo do campo elétrico e sua energia, a equação (8.29) nos leva à antiga idéia, proposta por Abraham-Lorentz [47], e mais elaborada na eletrodinâmica de Born-Infeld [31], de um elétron sendo uma partícula de energia finita, ou em termos contemporâneos, o sóliton, cuja energia pode ser manifestada completamente na forma de um campo. Essa idéia foi considerada de forma bem sucedida em uma tentativa de Infeld [48], trada como uma "curiosidade histórica" em [44], para determinar a constante de estrutura fina, após equacionar o parâmetro dimensional livre, inerente ao modelo de Born-Infeld e fixado pelo requisito que a massa do elétron deve ter origem puramente no campo, com seu valor correspondente na QED. Hoje em dia há pouca fundamentação para se acreditar que a massa do elétron seja oriunda apenas de seu campo eletromagnético sozinho, porque o elétron está envolvido em outras interações também. De qualquer forma, a questão da finitude da energia de uma carga puntiforme está estritamente atrelada ao efeito da não linearidade devida aos diagramas de Feynman, cuja lagrangeana efetiva regulariza as integrais envolvidas, eliminando a divergência (8.27), mesmo quando é minimal (diagrama de espalhamento fóton por fóton), representada pelo modelo quártico, na qual todos os outros modelos são redutíveis, quando se considera a constante de acoplamento pequena. Por outro lado, o elétron real, além de ser um monopolo elétrico, é também um dipolo magnético puntiforme, portanto espera-se que a energia associada ao campo magnético é esperada para contribuir à massa total [49]. Um problema mais desafiador é tomar as duas interações mútuas simultaneamente, para que, pelo menos, o termo proporcional a $\mathfrak{G}^{2}$ seja adicionado à lagrangeana (8.4). Essa discussão pode levar a possibilidades futuras, e gerar perspectivas de cálculos mais realísticos acerca da natureza do elétron. 


\section{Apêndice A}

\section{Derivadas variacionais}

\section{A.1 Séries de Taylor variacionais e resolução das equações de Euler-Lagrange perturbativas}

Dado o funcional $\Gamma=\int \mathcal{L}(\mathfrak{F}(x), \mathfrak{G}(x)) \mathrm{d}^{4} x$, podemos expandi-lo em séries de potências variacionais de $a=A-\mathcal{A}$, em torno do ponto $A=\mathcal{A}$ :

$$
\begin{aligned}
& \frac{\delta \Gamma}{\delta A^{\rho}(x)}=\left.\frac{\delta \Gamma}{\delta A^{\rho}(x)}\right|_{A=\mathcal{A}}+\left.\int \frac{\delta^{2} \Gamma}{\delta A^{\rho}(x) \delta A^{\alpha}\left(x^{\prime}\right)}\right|_{A=\mathcal{A}} a^{\alpha}\left(x^{\prime}\right) \mathrm{d}^{4} x^{\prime} \\
& +\left.\frac{1}{2} \iint \frac{\delta^{3} \Gamma}{\delta A^{\rho}(x) \delta A^{\alpha}\left(x^{\prime}\right) \delta A^{\beta}\left(x^{\prime \prime}\right)}\right|_{A=\mathcal{A}} a^{\alpha}\left(x^{\prime}\right) a^{\beta}\left(x^{\prime \prime}\right) \mathrm{d}^{4} x^{\prime} \mathrm{d}^{4} x^{\prime \prime} \\
& +\left.\frac{1}{6} \iint \frac{\delta^{4} \Gamma}{\delta A^{\rho}(x) \delta A^{\alpha}\left(x^{\prime}\right) \delta A^{\beta}\left(x^{\prime \prime}\right) \delta A^{\gamma}\left(x^{\prime \prime \prime}\right)}\right|_{A=\mathcal{A}} a^{\alpha}\left(x^{\prime}\right) a^{\beta}\left(x^{\prime \prime}\right) a^{\gamma}\left(x^{\prime \prime \prime}\right) \mathrm{d}^{4} x^{\prime} \mathrm{d}^{4} x^{\prime \prime} \mathrm{d}^{4} x^{\prime \prime \prime}+\ldots
\end{aligned}
$$

Logo, (3.3) se torna, lembrando que $S=\Gamma+S_{\text {free }}$,

$$
\begin{aligned}
& J_{\mu}(x)=\frac{\delta S_{\text {free }}}{\delta A^{\mu}(x)}-\left.\frac{\delta S_{\text {free }}}{\delta A^{\mu}(x)}\right|_{A=\mathcal{A}}+\left.\frac{\delta S}{\delta A^{\mu}(x)}\right|_{A=\mathcal{A}}+\left.\int \frac{\delta^{2} \Gamma}{\delta A^{\mu}(x) \delta A^{\tau}\left(x^{\prime}\right)}\right|_{A=\mathcal{A}} a^{\tau}\left(x^{\prime}\right) \mathrm{d}^{4} x^{\prime} \\
& +\left.\frac{1}{2} \iint \frac{\delta^{3} \Gamma}{\delta A^{\mu}(x) \delta A^{\tau}\left(x^{\prime}\right) \delta A^{\sigma}\left(x^{\prime \prime}\right)}\right|_{A=\mathcal{A}} a^{\tau}\left(x^{\prime}\right) a^{\sigma}\left(x^{\prime \prime}\right) \mathrm{d}^{4} x^{\prime} \mathrm{d}^{4} x^{\prime \prime}+\ldots
\end{aligned}
$$

É facil de mostrar que

$$
\frac{\delta S_{\text {free }}}{\delta A^{\rho}(x)}=\left[\square \eta_{\rho \nu}-\partial_{\nu} \partial_{\rho}\right] A^{\nu}(x)=\left[\square \eta_{\rho \nu}-\partial_{\nu} \partial_{\rho}\right] a^{\nu}(x)+\left.\frac{\delta S_{\text {free }}}{\delta A^{\rho}(x)}\right|_{A=\mathcal{A}},
$$

e desta forma obtemos (5.9):

$$
\begin{aligned}
& j_{\mu}(x)=\left[\square \eta_{\mu \tau}-\partial_{\mu} \partial_{\tau}\right] a^{\tau}(x)+\left.\int \mathrm{d}^{4} y \frac{\delta^{2} \Gamma}{\delta A^{\mu}(x) \delta A^{\tau}(y)}\right|_{A=\mathcal{A}} a^{\tau}(y) \\
& +\left.\frac{1}{2} \int \mathrm{d}^{4} y \mathrm{~d}^{4} u \frac{\delta^{3} \Gamma}{\delta A^{\mu}(x) \delta A^{\tau}(y) \delta A^{\sigma}(u)}\right|_{A=\mathcal{A}} a^{\tau}(y) a^{\sigma}(u) \\
& +\left.\frac{1}{6} \int \mathrm{d}^{4} y \mathrm{~d}^{4} u \mathrm{~d}^{4} v \frac{\delta^{4} \Gamma}{\delta A^{\mu}(x) \delta A^{\tau}(y) \delta A^{\sigma}(u) \delta A^{\rho}(v)}\right|_{A=\mathcal{A}} a^{\tau}(y) a^{\sigma}(u) a^{\rho}(v)+\ldots
\end{aligned}
$$


Para calcular as derivadas variacionais de uma ação $\Gamma$, vamos escrever a ação em termos da lagrangeana $\mathcal{L}$. E é justamente neste ponto que fazemos uso da aproximação infra-vermelho: esta lagrangeana depende apenas dos invariantes $\mathfrak{F}$ e $\mathfrak{G}$, e não de suas derivadas:

$$
\Gamma=\int \mathcal{L}(\mathfrak{F}(z), \mathfrak{G}(z)) \mathrm{d}^{4} z=\int \mathcal{L}(\mathfrak{F}(x), \mathfrak{G}(x)) \delta^{4}(x-z) \mathrm{d}^{4} x \mathrm{~d}^{4} z .
$$

De uma forma operacional, cada derivada variacional pode ser calculada por

$$
\frac{\delta \Gamma}{\delta A^{\mu}(x)}=\frac{\partial \mathcal{L}(\mathfrak{F}(x), \mathfrak{G}(x))}{\partial A^{\mu}(x)}-\partial_{\nu} \frac{\partial \mathcal{L}(\mathfrak{F}(x), \mathfrak{G}(x))}{\partial\left(\partial^{\nu} A^{\mu}(x)\right)} .
$$

É claro que $\mathcal{L}(\mathfrak{F}(x), \mathfrak{G}(x))$ não depende de $A^{\mu}(x)$, mas depende somente de suas derivadas $\partial^{\nu} A^{\mu}(x)$, porque ambos invariantes $\mathfrak{F}(x)$ e $\mathfrak{G}(x)$ dependem exclusivamente de $F_{\mu \nu}$. Portanto,

$$
\begin{aligned}
& \frac{\delta \Gamma}{\delta A^{\mu}(x)}=-\int \frac{\partial}{\partial z^{\nu}}\left[\frac{\partial \mathcal{L}(\mathfrak{F}(z), \mathfrak{G}(z))}{\partial\left(\partial^{\nu} A^{\mu}(z)\right)}\right] \delta^{4}(x-z) \mathrm{d}^{4} z \\
= & \int \frac{\partial \mathcal{L}(\mathfrak{F}(z), \mathfrak{G}(z))}{\partial\left(\partial^{\nu} A^{\mu}(z)\right)} \frac{\partial \delta^{4}(x-z)}{\partial z_{\nu}} \mathrm{d}^{4} z .
\end{aligned}
$$

Tendo em mende a dependência $\mathfrak{L}=\mathfrak{L}(\mathfrak{F}(z), \mathfrak{G}(z)), \mathfrak{F}=\mathfrak{F}(z)$, e $\mathfrak{G}=\mathfrak{G}(z)$, podemos calcular as seguintes derivadas:

$$
\begin{aligned}
\frac{\partial F_{\gamma \sigma}}{\partial\left(\partial^{\lambda} A^{\rho}\right)} & =\eta_{\gamma \lambda} \eta_{\rho \sigma}-\eta_{\sigma \lambda} \eta_{\gamma \rho}, \quad \frac{\partial \tilde{F}_{\gamma \sigma}}{\partial\left(\partial^{\lambda} A^{\rho}\right)}=\epsilon_{\lambda \rho \gamma \sigma}, \\
\frac{\partial}{\partial\left(\partial^{\lambda} A^{\rho}\right)} & =\frac{\partial \mathfrak{F}}{\partial\left(\partial^{\lambda} A^{\rho}\right)} \frac{\partial}{\partial \mathfrak{F}}+\frac{\partial \mathfrak{G}}{\partial\left(\partial^{\lambda} A^{\rho}\right)} \frac{\partial}{\partial \mathfrak{G}}=\frac{1}{4 \pi}\left(F_{\lambda \rho} \frac{\partial}{\partial \mathfrak{F}}+\tilde{F}_{\lambda \rho} \frac{\partial}{\partial \mathfrak{G}}\right) .
\end{aligned}
$$

Desta forma, podmos calcular as derivadas variacionais de quaisquer ordens da ação $\Gamma$ usando (A.2), que, lembrando das definições (3.5), (3.6), e (3.7), são os tensores de polarização, quando tomados em um ponto $A=\mathcal{A}$, calculados explicitamente a seguir usando as relações (A.4).

\section{A.2 Derivadas variacionais da ação efetiva}

Calculamos explicitamente as quatro primeiras derivadas variacionais de $\Gamma$ a partir de (A.3) e (A.4). Aqui omitimos a dependência $\mathfrak{L}=\mathfrak{L}(\mathfrak{F}(z), \mathfrak{G}(z))$.

\section{A.2.1 Primeira ordem}

A derivada variacional da ação efetiva é dada por:

$$
\frac{\delta \Gamma}{\delta A^{\mu}(x)}=\int\left(F_{\alpha \mu} \frac{\partial \mathcal{L}}{\partial \mathfrak{F}}+\tilde{F}_{\alpha \mu} \frac{\partial \mathcal{L}}{\partial \mathfrak{G}}\right) \frac{\partial}{\partial z_{\alpha}} \delta^{4}(x-z) \mathrm{d}^{4} z .
$$




\section{A.2.2 Segunda ordem}

A resposta linear, que é a segunda derivada variacional da ação efetiva, é dada por:

$$
\begin{aligned}
& \frac{\delta^{2} \Gamma}{\delta A^{\mu}(x) \delta A^{\tau}(y)}=\int \mathrm{d}^{4} z\left[\frac{\partial \mathfrak{L}}{\partial \mathfrak{F}}\left(\eta_{\mu \tau} \eta_{\alpha \beta}-\eta_{\mu \beta} \eta_{\alpha \tau}\right)+\right. \\
& +\frac{\partial \mathfrak{L}}{\partial \mathfrak{G}} \epsilon_{\alpha \mu \beta \tau}+\frac{\partial^{2} \mathfrak{L}}{\partial \mathfrak{F}^{2}} F_{\alpha \mu} F_{\beta \tau}+\frac{\partial^{2} \mathfrak{L}}{\partial \mathfrak{G}^{2}} \tilde{F}_{\alpha \mu} \tilde{F}_{\beta \tau}+ \\
& \left.+\frac{\partial^{2} \mathfrak{L}}{\partial \mathfrak{F} \partial \mathfrak{G}}\left(F_{\alpha \mu} \tilde{F}_{\beta \tau}+\tilde{F}_{\alpha \mu} F_{\beta \tau}\right)\right]\left(\frac{\partial}{\partial z_{\alpha}} \delta^{4}(x-z)\right)\left(\frac{\partial}{\partial z_{\beta}} \delta^{4}(y-z)\right) .
\end{aligned}
$$

\section{A.2.3 Terceira ordem}

A resposta não linear em campo de fundo, que é a terceira derivada variacional da ação efetiva é (note a dependência do tensor $F_{\mu \nu}$ em todos os termos, que é zero quando não há campo externo de fundo):

$$
\begin{aligned}
& \frac{\delta^{3} \Gamma}{\delta A^{\mu}(x) \delta A^{\tau}(y) \delta A^{\sigma}(u)}= \\
& \int \mathrm{d}^{4} z\left\{\left(\eta_{\mu \tau} \eta_{\alpha \beta}-\eta_{\mu \beta} \eta_{\alpha \tau}\right)\left(\frac{\partial^{2} \mathfrak{L}}{\partial \mathfrak{F}^{2}} F_{\gamma \sigma}+\frac{\partial^{2} \mathfrak{L}}{\partial \mathfrak{G} \partial \mathfrak{F}} \tilde{F}_{\gamma \sigma}\right) \frac{\partial}{\partial z_{\gamma}} \delta^{4}(u-z)+\right. \\
& +\epsilon_{\alpha \mu \beta \tau}\left(\frac{\partial^{2} \mathfrak{L}}{\partial \mathfrak{F} \partial \mathfrak{G}} F_{\gamma \sigma}+\frac{\partial^{2} \mathfrak{L}}{\partial \mathfrak{G}^{2}} \tilde{F}_{\gamma \sigma}\right) \frac{\partial}{\partial z_{\gamma}} \delta^{4}(u-z) \\
& +\left(\frac{\partial^{3} \mathfrak{L}}{\partial \mathfrak{F}^{3}} F_{\alpha \mu} F_{\beta \tau} F_{\gamma \sigma}+\frac{\partial^{3} \mathfrak{L}}{\partial \mathfrak{G} \partial \mathfrak{F}^{2}} F_{\alpha \mu} F_{\beta \tau} \tilde{F}_{\gamma \sigma}\right) \frac{\partial}{\partial z_{\gamma}} \delta^{4}(u-z)+ \\
& +\frac{\partial^{2} \mathfrak{L}}{\partial \mathfrak{F}^{2}}\left[F_{\alpha \mu}\left(\eta_{\tau \sigma} \frac{\partial}{\partial z^{\beta}}-\eta_{\beta \sigma} \frac{\partial}{\partial z^{\tau}}\right)+F_{\beta \tau}\left(\eta_{\mu \sigma} \frac{\partial}{\partial z^{\alpha}}-\eta_{\alpha \sigma} \frac{\partial}{\partial z^{\mu}}\right)\right] \delta^{4}(u-z)+ \\
& +\tilde{F}_{\alpha \mu} \tilde{F}_{\beta \tau}\left(\frac{\partial^{3} \mathfrak{L}}{\partial \mathfrak{F} \partial \mathfrak{G}^{2}} F_{\gamma \sigma}+\frac{\partial^{3} \mathfrak{L}}{\partial \mathfrak{G}^{3}} \tilde{F}_{\gamma \sigma}\right) \frac{\partial}{\partial z_{\gamma}} \delta^{4}(u-z)+ \\
& +\frac{\partial^{2} \mathfrak{L}}{\partial \mathfrak{G}^{2}}\left(\tilde{F}_{\alpha \mu} \epsilon_{\beta \tau \gamma \sigma}+\tilde{F}_{\beta \tau} \epsilon_{\alpha \mu \gamma \sigma}\right) \frac{\partial}{\partial z_{\gamma}} \delta^{4}(u-z)+ \\
& +\frac{\partial^{2} \mathfrak{L}}{\partial \mathfrak{F} \partial \mathfrak{G}}\left[F_{\alpha \mu} \epsilon_{\beta \tau \gamma \sigma} \frac{\partial}{\partial z_{\gamma}}+\tilde{F}_{\beta \tau}\left(\eta_{\mu \sigma} \frac{\partial}{\partial z^{\alpha}}-\eta_{\alpha \sigma} \frac{\partial}{\partial z^{\mu}}\right)+\tilde{F}_{\alpha \mu}\left(\eta_{\tau \sigma} \frac{\partial}{\partial z^{\beta}}-\eta_{\beta \sigma} \frac{\partial}{\partial z^{\tau}}\right)+\right. \\
& \left.+F_{\beta \tau} \epsilon_{\alpha \mu \gamma \sigma} \frac{\partial}{\partial z_{\gamma}}\right] \delta^{4}(u-z)+ \\
& +\left(F_{\alpha \mu} \tilde{F}_{\beta \tau}+\tilde{F}_{\alpha \mu} F_{\beta \tau}\right)\left(\frac{\partial^{3} \mathfrak{L}}{\partial \mathfrak{F}^{2} \partial \mathfrak{G}} F_{\gamma \sigma}+\frac{\partial^{3} \mathfrak{L}}{\partial \mathfrak{F} \partial \mathfrak{G}^{2}} \tilde{F}_{\gamma \sigma}\right) \\
& \left.\frac{\partial}{\partial z_{\gamma}} \delta^{4}(u-z)\right\}\left(\frac{\partial}{\partial z_{\alpha}} \delta^{4}(x-z)\right)\left(\frac{\partial}{\partial z_{\beta}} \delta^{4}(y-z)\right) \cdot
\end{aligned}
$$

\section{A.2.4 Quarta ordem}

E finalmente, a resposta não linear do vácuo, que é a quarta derivada variacional da ação efetiva, é dada por (note que há termos que não dependem de $F_{\mu \nu}$, ou seja, existe mesmo no vácuo, e por isso 
o nome):

$$
\begin{aligned}
& \frac{\delta^{4} \Gamma}{\delta A^{\mu}(x) \delta A^{\tau}(y) \delta A^{\sigma}(u) \delta A^{\rho}(v)}= \\
& =\int \mathrm{d}^{4} z\left\{\frac { \partial ^ { 2 } \mathfrak { L } } { \partial \mathfrak { F } ^ { 2 } } \left[\left(\eta_{\alpha \lambda} \eta_{\rho \mu}-\eta_{\mu \lambda} \eta_{\alpha \rho}\right)\left(\eta_{\tau \sigma} \eta_{\beta \gamma}-\eta_{\beta \sigma} \eta_{\tau \gamma}\right)\right.\right. \\
& \left.+\left(\eta_{\beta \lambda} \eta_{\rho \tau}-\eta_{\tau \lambda} \eta_{\beta \rho}\right)\left(\eta_{\mu \sigma} \eta_{\alpha \gamma}-\eta_{\alpha \sigma} \eta_{\mu \gamma}\right)+\left(\eta_{\gamma \lambda} \eta_{\rho \sigma}-\eta_{\sigma \lambda} \eta_{\gamma \rho}\right)\left(\eta_{\mu \tau} \eta_{\alpha \beta}-\eta_{\mu \beta} \eta_{\alpha \tau}\right)\right] \\
& +\frac{\partial^{2} \mathfrak{L}}{\partial \mathfrak{F} \partial \mathfrak{G}}\left[\left(\eta_{\mu \tau} \eta_{\alpha \beta}-\eta_{\mu \beta} \eta_{\alpha \tau}\right) \epsilon_{\lambda \rho \gamma \sigma}+\left(\eta_{\gamma \lambda} \eta_{\rho \sigma}-\eta_{\sigma \lambda} \eta_{\gamma \rho}\right) \epsilon_{\alpha \mu \beta \tau}+\left(\eta_{\alpha \lambda} \eta_{\rho \mu}-\eta_{\mu \lambda} \eta_{\alpha \rho}\right) \epsilon_{\beta \tau \gamma \sigma}\right. \\
& \left.+\left(\eta_{\beta \lambda} \eta_{\rho \tau}-\eta_{\tau \lambda} \eta_{\beta \rho}\right) \epsilon_{\alpha \mu \gamma \sigma}+\left(\eta_{\mu \sigma} \eta_{\alpha \gamma}-\eta_{\alpha \sigma} \eta_{\mu \sigma}\right) \epsilon_{\lambda \rho \beta \tau}+\left(\eta_{\tau \sigma} \eta_{\beta \gamma}-\eta_{\beta \sigma} \eta_{\tau \gamma}\right) \epsilon_{\lambda \rho \alpha \mu}\right] \\
& +\frac{\partial^{2} \mathfrak{L}}{\partial \mathfrak{G}^{2}}\left(\epsilon_{\alpha \mu \beta \tau} \epsilon_{\lambda \rho \gamma \sigma}+\epsilon_{\lambda \rho \alpha \mu} \epsilon_{\beta \tau \gamma \sigma}+\epsilon_{\lambda \rho \beta \tau} \epsilon_{\alpha \mu \gamma \sigma}\right) \\
& +\frac{\partial^{3} \mathfrak{L}}{\partial \mathfrak{F}^{3}}\left[F_{\alpha \mu} F_{\beta \tau}\left(\eta_{\gamma \lambda} \eta_{\rho \sigma}-\eta_{\sigma \lambda} \eta_{\gamma \rho}\right)+F_{\beta \tau} F_{\gamma \sigma}\left(\eta_{\alpha \lambda} \eta_{\rho \mu}-\eta_{\mu \lambda} \eta_{\alpha \rho}\right)+F_{\alpha \mu} F_{\gamma \sigma}\left(\eta_{\beta \lambda} \eta_{\rho \tau}-\eta_{\tau \lambda} \eta_{\beta \rho}\right)\right. \\
& \left.+F_{\alpha \mu} F_{\lambda \rho}\left(\eta_{\tau \sigma} \eta_{\beta \gamma}-\eta_{\beta \sigma} \eta_{\tau \gamma}\right)+F_{\beta \tau} F_{\lambda \rho}\left(\eta_{\mu \sigma} \eta_{\alpha \gamma}-\eta_{\alpha \sigma} \eta_{\mu \gamma}\right)+F_{\gamma \sigma} F_{\lambda \rho}\left(\eta_{\mu \tau} \eta_{\alpha \beta}-\eta_{\mu \beta} \eta_{\alpha \tau}\right)\right] \\
& +\frac{\partial^{3} \mathfrak{L}}{\partial \mathfrak{F}^{2} \partial \mathfrak{G}}\left[\left(F_{\alpha \mu} \tilde{F}_{\beta \tau}+\tilde{F}_{\alpha \mu} F_{\beta \tau}\right)\left(\eta_{\gamma \lambda} \eta_{\rho \sigma}-\eta_{\sigma \lambda} \eta_{\gamma \rho}\right)+\left(F_{\beta \tau} \tilde{F}_{\gamma \sigma}+F_{\gamma \sigma} \tilde{F}_{\beta \tau}\right)\left(\eta_{\alpha \lambda} \eta_{\rho \mu}-\eta_{\mu \lambda} \eta_{\alpha \rho}\right)\right. \\
& +\left(F_{\gamma \sigma} \tilde{F}_{\lambda \rho}+\tilde{F}_{\gamma \sigma} F_{\lambda \rho}\right)\left(\eta_{\mu \tau} \eta_{\alpha \beta}-\eta_{\mu \beta} \eta_{\alpha \tau}\right)+\left(F_{\alpha \mu} \tilde{F}_{\gamma \sigma}+F_{\gamma \sigma} \tilde{F}_{\alpha \mu}\right)\left(\eta_{\beta \lambda} \eta_{\rho \tau}-\eta_{\tau \lambda} \eta_{\beta \rho}\right) \\
& +F_{\alpha \mu} \tilde{F}_{\lambda \rho}\left(\eta_{\tau \sigma} \eta_{\beta \gamma}-\eta_{\beta \sigma} \eta_{\tau \gamma}\right)+F_{\beta \tau} \tilde{F}_{\lambda \rho}\left(\eta_{\mu \sigma} \eta_{\alpha \gamma}-\eta_{\alpha \sigma} \eta_{\mu \gamma}\right) \\
& \left.+F_{\alpha \mu} F_{\beta \tau} \epsilon_{\lambda \rho \gamma \sigma}+F_{\beta \tau} F_{\gamma \sigma} \epsilon_{\lambda \rho \alpha \mu}+F_{\gamma \sigma} F_{\lambda \rho} \epsilon_{\alpha \mu \beta \tau}+F_{\alpha \mu} \epsilon_{\lambda \rho \beta \tau}\right] \\
& +\frac{\partial^{3} \mathfrak{L}}{\partial \mathfrak{F} \partial \mathfrak{G}^{2}}\left[\tilde{F}_{\alpha \mu} \tilde{F}_{\beta \tau}\left(\eta_{\gamma \lambda} \eta_{\rho \sigma}-\eta_{\sigma \lambda} \eta_{\gamma \rho}\right)+\tilde{F}_{\alpha \mu} \tilde{F}_{\lambda \rho}\left(\eta_{\tau \sigma} \eta_{\beta \gamma}-\eta_{\beta \sigma} \eta_{\tau \gamma}\right)\right. \\
& +\tilde{F}_{\beta \tau} \tilde{F}_{\lambda \rho}\left(\eta_{\mu \sigma} \eta_{\alpha \gamma}-\eta_{\alpha \sigma} \eta_{\mu \sigma}\right)+\tilde{F}_{\gamma \sigma} \tilde{F}_{\lambda \rho}\left(\eta_{\mu \tau} \eta_{\alpha \beta}-\eta_{\mu \beta} \eta_{\alpha \tau}\right)+\left(F_{\gamma \sigma} \tilde{F}_{\lambda \rho}+\tilde{F}_{\gamma \sigma} F_{\lambda \rho}\right) \epsilon_{\alpha \mu \beta \tau} \\
& \left.+F_{\gamma \sigma} \tilde{F}_{\alpha \mu} \epsilon_{\lambda \rho \beta \tau}+F_{\gamma \sigma} \tilde{F}_{\beta \tau} \epsilon_{\lambda \rho \alpha \mu}+F_{\alpha \mu} \tilde{F}_{\lambda \rho} \epsilon_{\beta \tau \gamma \sigma}+F_{\beta \tau} \tilde{F}_{\lambda \rho} \epsilon_{\alpha \mu \gamma \sigma}\right]+\frac{\partial^{4} \mathfrak{L}}{\partial \mathfrak{F}^{4}}\left(F_{\alpha \mu} F_{\beta \tau} F_{\gamma \sigma} F_{\lambda \rho}\right) \\
& +\frac{\partial^{4} \mathfrak{L}}{\partial \mathfrak{F}^{3} \partial \mathfrak{G}}\left(F_{\alpha \mu} F_{\beta \tau} F_{\gamma \sigma} \tilde{F}_{\lambda \rho}+F_{\alpha \mu} F_{\beta \tau} F_{\lambda \rho} \tilde{F}_{\gamma \sigma}+F_{\alpha \mu} F_{\gamma \sigma} F_{\lambda \rho} \tilde{F}_{\beta \tau}+F_{\beta \tau} F_{\gamma \sigma} F_{\lambda \rho} \tilde{F}_{\alpha \mu}\right) \\
& +\frac{\partial^{4} \mathfrak{L}}{\partial \mathfrak{F}^{2} \partial \mathfrak{G}^{2}}\left(F_{\gamma \sigma} F_{\lambda \rho} \tilde{F}_{\alpha \mu} \tilde{F}_{\beta \tau}+F_{\alpha \mu} F_{\beta \tau} \tilde{F}_{\gamma \sigma} \tilde{F}_{\lambda \rho}+F_{\alpha \mu} F_{\gamma \sigma} \tilde{F}_{\beta \tau} \tilde{F}_{\lambda \rho}\right. \\
& \left.+F_{\alpha \mu} F_{\lambda \rho} \tilde{F}_{\gamma \sigma} \tilde{F}_{\beta \tau}+F_{\beta \tau} F_{\lambda \rho} \tilde{F}_{\gamma \sigma} \tilde{F}_{\alpha \mu}+F_{\gamma \sigma} F_{\beta \tau} \tilde{F}_{\alpha \mu} \tilde{F}_{\lambda \rho}\right) \\
& +\frac{\partial^{4} \mathfrak{L}}{\partial \mathfrak{F} \partial \mathfrak{G}^{3}}\left(F_{\alpha \mu} \tilde{F}_{\beta \tau} \tilde{F}_{\gamma \sigma} \tilde{F}_{\lambda \rho}+F_{\beta \tau} \tilde{F}_{\alpha \mu} \tilde{F}_{\gamma \sigma} \tilde{F}_{\lambda \rho}+F_{\gamma \sigma} \tilde{F}_{\alpha \mu} \tilde{F}_{\beta \tau} \tilde{F}_{\lambda \rho}+F_{\lambda \rho} \tilde{F}_{\alpha \mu} \tilde{F}_{\beta \tau} \tilde{F}_{\gamma \sigma}\right) \\
& \left.+\frac{\partial^{4} \mathfrak{L}}{\partial \mathfrak{G}^{4}} \tilde{F}_{\alpha \mu} \tilde{F}_{\beta \tau} \tilde{F}_{\gamma \sigma} \tilde{F}_{\lambda \rho}\right\} \frac{\partial \delta^{4}(x-z)}{\partial z_{\alpha}} \frac{\partial \delta^{4}(y-z)}{\partial z_{\beta}} \frac{\partial \delta^{4}(u-z)}{\partial z_{\gamma}} \frac{\partial \delta^{4}(v-z)}{\partial z_{\lambda}} .
\end{aligned}
$$




\section{Apêndice B}

\section{Existência das soluções das equações de Maxwell extacionárias: Os operadores de projeção}

\section{B.1 Operador de projeção longitudinal}

Seja F (r) um campo vetorial. Então vamos mostrar que sua projeção longitudinal $\mathbf{F}^{\|}$(r) é dada, em coordenadas cartesianas, por

$$
F_{i}^{\|}(\mathbf{r})=-\frac{\nabla_{i} \nabla_{j}}{4 \pi} \int \frac{F_{j}\left(\mathbf{r}^{\prime}\right)}{\left|\mathbf{r}-\mathbf{r}^{\prime}\right|} \mathrm{d} \mathbf{r}^{\prime}
$$

O operador diferencial $\nabla_{j}$ atua somente na função de Green

$$
F_{i}^{\|}(\mathbf{r})=-\frac{\nabla_{i}}{4 \pi} \int F_{j}\left(\mathbf{r}^{\prime}\right) \nabla_{j}\left(\frac{1}{\left|\mathbf{r}-\mathbf{r}^{\prime}\right|}\right) \mathrm{d} \mathbf{r}^{\prime} .
$$

Pela regra da cadeia, podemos trocar $\nabla_{j} \leftrightarrow-\nabla_{j}^{\prime}$

$$
F_{i}^{\|}(\mathbf{r})=\frac{\nabla_{i}}{4 \pi} \int F_{j}\left(\mathbf{r}^{\prime}\right) \nabla_{j}^{\prime}\left(\frac{1}{\left|\mathbf{r}-\mathbf{r}^{\prime}\right|}\right) \mathrm{d} \mathbf{r}^{\prime} .
$$

Por integração por partes, supondo que $\lim _{r \rightarrow \infty} r \mathbf{F}(\mathbf{r})=0$,

$$
F_{i}^{\|}(\mathbf{r})=-\frac{\nabla_{i}}{4 \pi} \int \frac{\nabla_{j}^{\prime} F_{j}\left(\mathbf{r}^{\prime}\right)}{\left|\mathbf{r}-\mathbf{r}^{\prime}\right|} \mathrm{d} \mathbf{r}^{\prime}
$$

Enfim, na forma vetorial,

$$
\mathbf{F}^{\|}(\mathbf{r})=-\frac{1}{4 \pi} \nabla \int \frac{\nabla^{\prime} \cdot \mathbf{F}\left(\mathbf{r}^{\prime}\right)}{\left|\mathbf{r}-\mathbf{r}^{\prime}\right|} \mathrm{d} \mathbf{r}^{\prime}
$$

Para notar que $\mathbf{F}^{\|}(\mathbf{r})$, dado por (B.1), é de fato a projeção longitudinal de $\mathbf{F}$ (r), basta notar que

$$
\begin{aligned}
\nabla \times \mathbf{F}^{\|}(\mathbf{r}) & =\mathbf{0} \\
\boldsymbol{\nabla} \cdot \mathbf{F}^{\|}(\mathbf{r}) & =\boldsymbol{\nabla} \cdot \mathbf{F}(\mathbf{r}) .
\end{aligned}
$$


A primeira equação é garantida pelo fato de que o rotacional de um gradiente de um campo escalar qualquer é sempre zero, e a segunda equação

$$
\begin{aligned}
& \boldsymbol{\nabla} \cdot\left(-\frac{1}{4 \pi} \boldsymbol{\nabla} \int \frac{\boldsymbol{\nabla}^{\prime} \cdot \mathbf{F}\left(\mathbf{r}^{\prime}\right)}{\left|\mathbf{r}-\mathbf{r}^{\prime}\right|} \mathrm{d} \mathbf{r}^{\prime}\right)=-\frac{1}{4 \pi} \int \nabla^{2} \frac{\nabla^{\prime} \cdot \mathbf{F}\left(\mathbf{r}^{\prime}\right)}{\left|\mathbf{r}-\mathbf{r}^{\prime}\right|} \mathrm{d} \mathbf{r}^{\prime} \\
= & \int \boldsymbol{\nabla}^{\prime} \cdot \mathbf{F}\left(\mathbf{r}^{\prime}\right) \delta\left(\mathbf{r}-\mathbf{r}^{\prime}\right) \mathrm{d} \mathbf{r}^{\prime}=\boldsymbol{\nabla} \cdot \mathbf{F}(\mathbf{r}),
\end{aligned}
$$

é devida à relação

$$
\nabla^{2}\left(\frac{1}{\left|\mathbf{r}-\mathbf{r}^{\prime}\right|}\right)=-4 \pi \delta\left(\mathbf{r}-\mathbf{r}^{\prime}\right)
$$

\section{B.2 Operador de projeção transversal}

Devido a complementaridade, a projeção transversal de um campo vetorial $\mathbf{F}(\mathbf{r})$, denotada por $\mathbf{F}^{\perp}(\mathbf{r})$, é tal que sua soma com $\mathbf{F}^{\|}(\mathbf{r})$, dada por (B.1), é o próprio campo $\mathbf{F}(\mathbf{r})$, i.e., $\mathbf{F}(\mathbf{r})=$ $\mathbf{F}^{\|}(\mathbf{r})+\mathbf{F}^{\perp}(\mathbf{r})$ :

$$
\mathbf{F}^{\perp}(\mathbf{r})=\mathbf{F}(\mathbf{r})+\frac{1}{4 \pi} \nabla \int \frac{\nabla^{\prime} \cdot \mathbf{F}\left(\mathbf{r}^{\prime}\right)}{\left|\mathbf{r}-\mathbf{r}^{\prime}\right|} \mathrm{d} \mathbf{r}^{\prime} .
$$

Para notar que $\mathbf{F}^{\perp}(\mathbf{r})$, dado por (B.4), é de fato a projeção transversal de $\mathbf{F}$ (r), basta notar que

$$
\begin{aligned}
\nabla \cdot \mathbf{F}^{\perp}(\mathbf{r}) & =0 \\
\nabla \times \mathbf{F}^{\perp}(\mathbf{r}) & =\boldsymbol{\nabla} \times \mathbf{F}(\mathbf{r}),
\end{aligned}
$$

pois o rotacional de um gradiente de um campo escalar qualquer (segundo termo) é sempre zero, e $\boldsymbol{\nabla} \cdot \mathbf{F}^{\perp}(\mathbf{r})=\boldsymbol{\nabla} \cdot \mathbf{F}(\mathbf{r})-\boldsymbol{\nabla} \cdot \mathbf{F}^{\|}(\mathbf{r})=0$, devido à segunda equação de (B.2).

Há uma expressão alternativa para o campo $\mathbf{F}^{\perp}(\mathbf{r})$ que permite definir seu potencial. É dada por

$$
\mathbf{F}^{\perp}(\mathbf{r})=\frac{1}{4 \pi} \nabla \times \int \frac{\nabla^{\prime} \times \mathbf{F}\left(\mathbf{r}^{\prime}\right)}{\left|\mathbf{r}-\mathbf{r}^{\prime}\right|} \mathrm{d} \mathbf{r}^{\prime}
$$

Para mostrar que (B.6), satisfaz (B.5), basta notar que a primeira equação é verificada porque o divergente de um rotacional de um campo vetorial qualquer é sempre zero.

Mostremos agora a segunda equação. Dado um campo vetorial $\mathbf{A}(\mathbf{r})$, pela identidade vetorial $\boldsymbol{\nabla} \times[\boldsymbol{\nabla} \times \mathbf{A}(\mathbf{r})]=\boldsymbol{\nabla}(\boldsymbol{\nabla} \cdot \mathbf{A}(\mathbf{r}))-\boldsymbol{\nabla}^{2} \mathbf{A}(\mathbf{r})$

$$
\boldsymbol{\nabla} \times \mathbf{F}^{\perp}(\mathbf{r})=\frac{1}{4 \pi} \boldsymbol{\nabla}\left[\boldsymbol{\nabla} \cdot \int \frac{\boldsymbol{\nabla}^{\prime} \times \mathbf{F}\left(\mathbf{r}^{\prime}\right)}{\left|\mathbf{r}-\mathbf{r}^{\prime}\right|} \mathrm{d} \mathbf{r}^{\prime}\right]-\frac{1}{4 \pi} \nabla^{2} \int \frac{\boldsymbol{\nabla}^{\prime} \times \mathbf{F}\left(\mathbf{r}^{\prime}\right)}{\left|\mathbf{r}-\mathbf{r}^{\prime}\right|} \mathrm{d} \mathbf{r}^{\prime} .
$$

Aplicando o divergente no integrando do primeiro termo, e atuando o laplaciano na função de Green no segundo termo, usando (B.3),

$$
\boldsymbol{\nabla} \times \mathbf{F}^{\perp}(\mathbf{r})=\frac{1}{4 \pi} \boldsymbol{\nabla}\left[\int\left[\nabla^{\prime} \times \mathbf{F}\left(\mathbf{r}^{\prime}\right)\right] \cdot \nabla\left(\frac{1}{\left|\mathbf{r}-\mathbf{r}^{\prime}\right|}\right) \mathrm{d} \mathbf{r}^{\prime}\right]+\int\left[\nabla^{\prime} \times \mathbf{F}\left(\mathbf{r}^{\prime}\right)\right] \delta\left(\mathbf{r}-\mathbf{r}^{\prime}\right) \mathrm{d} \mathbf{r}^{\prime} .
$$

Trocando $\nabla \leftrightarrow-\nabla^{\prime}$ e resolvendo a segunda integral obtemos

$$
\boldsymbol{\nabla} \times \mathbf{F}^{\perp}(\mathbf{r})=-\frac{1}{4 \pi} \boldsymbol{\nabla}\left[\int\left[\boldsymbol{\nabla}^{\prime} \times \mathbf{F}\left(\mathbf{r}^{\prime}\right)\right] \cdot \nabla^{\prime}\left(\frac{1}{\left|\mathbf{r}-\mathbf{r}^{\prime}\right|}\right) \mathrm{d} \mathbf{r}^{\prime}\right]+\nabla \times \mathbf{F}(\mathbf{r}) .
$$


Usando a identidade vetorial $\mathbf{A}(\mathbf{r}) \cdot \boldsymbol{\nabla} \psi(\mathbf{r})=\psi(\mathbf{r}) \boldsymbol{\nabla} \cdot \mathbf{A}(\mathbf{r})-\boldsymbol{\nabla} \cdot[\psi(\mathbf{r}) \mathbf{A}(\mathbf{r})]$, dado um campo escalar $\psi(\mathbf{r})$,

$$
\boldsymbol{\nabla} \times \mathbf{F}^{\perp}(\mathbf{r})=-\frac{1}{4 \pi} \boldsymbol{\nabla}\left[\int \frac{\boldsymbol{\nabla}^{\prime} \cdot\left[\boldsymbol{\nabla}^{\prime} \times \mathbf{F}\left(\mathbf{r}^{\prime}\right)\right]}{\left|\mathbf{r}-\mathbf{r}^{\prime}\right|} \mathrm{d} \mathbf{r}^{\prime}\right]+\frac{1}{4 \pi} \boldsymbol{\nabla}\left[\int \nabla^{\prime} \cdot\left(\frac{\nabla^{\prime} \times \mathbf{F}\left(\mathbf{r}^{\prime}\right)}{\left|\mathbf{r}-\mathbf{r}^{\prime}\right|}\right) \mathrm{d} \mathbf{r}^{\prime}\right]+\boldsymbol{\nabla} \times \mathbf{F}(\mathbf{r}) .
$$

A primeira integral é zero porque o divergente do rotacional de um campo vetorial qualquer é nulo, e a segunda integral é zero pelo teorema de Gauss:

$$
\int \boldsymbol{\nabla}^{\prime} \cdot\left(\frac{\boldsymbol{\nabla}^{\prime} \times \mathbf{F}\left(\mathbf{r}^{\prime}\right)}{\left|\mathbf{r}-\mathbf{r}^{\prime}\right|}\right) \mathrm{d} \mathbf{r}^{\prime}=\oint \frac{\nabla^{\prime} \times \mathbf{F}\left(\mathbf{r}^{\prime}\right)}{\left|\mathbf{r}-\mathbf{r}^{\prime}\right|} \cdot \mathrm{d} \mathbf{S}^{\prime}=0,
$$

se respeitada a condição $\lim _{r \rightarrow \infty} r \mathbf{F}(\mathbf{r})=0$.

Portanto, de fato,

$$
\boldsymbol{\nabla} \times \mathbf{F}^{\perp}(\mathbf{r})=\boldsymbol{\nabla} \times \mathbf{F}(\mathbf{r})
$$

q.e.d. 


\section{Apêndice $\mathrm{C}$}

\section{Unicidade das soluções das equações de Maxwell estacionárias}

Admitindo a existência de um campo $\mathbf{F}$ (r) que satisfaz as equações

$$
\begin{aligned}
\boldsymbol{\nabla} \cdot \mathbf{F}(\mathbf{r}) & =C(\mathbf{r}), \\
\boldsymbol{\nabla} \times \mathbf{F}(\mathbf{r}) & =\mathbf{D}(\mathbf{r}),
\end{aligned}
$$

dadas as fontes $C(\mathbf{r})$ e $\mathbf{D}(\mathbf{r})$, tais que $\lim _{r \rightarrow \infty} r^{2} C(\mathbf{r})=0$ e $\lim _{r \rightarrow \infty} r^{2} \mathbf{D}(\mathbf{r})=\mathbf{0}$, e sujeito à condição

$$
\lim _{r \rightarrow \infty} r \mathbf{F}(\mathbf{r})=0,
$$

vamos demonstrar que $\mathbf{F}$ (r) é único. De fato, seja $\mathbf{F}_{0}(\mathbf{r})$ outra solução para (C.1), $\log$ o $\boldsymbol{\nabla} \times \mathbf{F}_{0}(\mathbf{r})=$ $\mathbf{C}(\mathbf{r})$, e $\boldsymbol{\nabla} \cdot \mathbf{F}_{0}(\mathbf{r})=\mathbf{D}(\mathbf{r})$. Vamos definir $\mathbf{V}(\mathbf{r})=\mathbf{F}(\mathbf{r})-\mathbf{F}_{0}(\mathbf{r})$, logo

$$
[\boldsymbol{\nabla} \times \mathbf{V}(\mathbf{r})]=\left[\boldsymbol{\nabla} \times\left(\mathbf{F}(\mathbf{r})-\mathbf{F}_{0}(\mathbf{r})\right)\right]=\mathbf{C}(\mathbf{r})-\mathbf{C}(\mathbf{r})=0,
$$

e

$$
\boldsymbol{\nabla} \cdot \mathbf{V}(\mathbf{r})=\boldsymbol{\nabla} \cdot\left(\mathbf{F}(\mathbf{r})-\mathbf{F}_{0}(\mathbf{r})\right)=\mathbf{D}(\mathbf{r})-\mathbf{D}(\mathbf{r})=0 .
$$

(C.3) nos permite definir um escalar $\phi(\mathbf{r})$ tal que $\mathbf{V}(\mathbf{r})=-\boldsymbol{\nabla} \phi(\mathbf{r})$, e substituindo isto em (C.4),

$$
\boldsymbol{\nabla} \cdot \mathbf{V}(\mathbf{r})=-\nabla^{2} \phi(\mathbf{r})=0 .
$$

Fazendo-se uso da identidade $\boldsymbol{\nabla} \cdot(\phi \boldsymbol{\nabla} \phi)=\phi \nabla^{2} \phi+(\nabla \phi)^{2}$, integramos sobre uma esfera de volume $V$, e superfície $S$, cujo raio tende ao infinito:

$$
\int_{V} \boldsymbol{\nabla} \cdot(\phi \boldsymbol{\nabla} \phi) \mathrm{d} \mathbf{r}^{\prime}=\int_{V} \phi \nabla^{2} \phi \mathrm{d} \mathbf{r}^{\prime}+\int_{V}(\nabla \phi)^{2} \mathrm{~d} \mathbf{r}^{\prime} .
$$

Pelo teorema de Gauss, a primeira integral é dupla na superfície fechada de $V$ :

$$
\oint_{\sigma[V]}(\phi \boldsymbol{\nabla} \phi) \mathrm{d} \mathbf{a}=\int_{V} \phi \nabla^{2} \phi \mathrm{d} \mathbf{r}^{\prime}+\int_{V}(\nabla \phi)^{2} \mathrm{~d} \mathbf{r}^{\prime} .
$$

A condição (C.2) impõe que a primeira integral vai a zero, bem como a segunda integral, devido a (C.5). Lembrando que $\nabla \phi=-\mathbf{V}(\mathbf{r})$,

$$
\int_{V}[\mathbf{V}(\mathbf{r})]^{2} \mathrm{~d} \mathbf{r}^{\prime}=0 .
$$

Esta igualdade deve ser zero para qualquer volume $V, \log \mathbf{F}(\mathbf{r})-\mathbf{F}_{0}(\mathbf{r})=\mathbf{V}(\mathbf{r})=0$. Portanto $\mathbf{F}_{0}(\mathbf{r})=\mathbf{F}(\mathbf{r})$, e $\mathbf{F}(\mathbf{r})$ é a solução única, q.e.d. 


\section{Apêndice D}

\section{Solução de equações cúbicas: A fórmula de Tartaglia-Cardano}

Calculemos a solução analítica para a equação:

$$
\mathbf{E}(\mathbf{r})=\mathbf{E}^{\operatorname{lin}}(\mathbf{r})-\frac{\gamma}{2} \mathbf{E}(\mathbf{r}) E^{2}(r) .
$$

Como $\mathbf{E}(\mathbf{r})=E(r) \mathbf{e}_{r}$ e $\mathbf{E}^{\operatorname{lin}}(\mathbf{r})=E_{\text {lin }}(r) \mathbf{e}_{r}$,

$$
E=E_{\text {lin }}-\frac{\gamma}{2} E^{3}
$$

Vamos subdividir o campo $E$ em duas partes:

$$
E=E^{+}+E^{-} .
$$

tais que:

$$
E^{+} E^{-}=-\frac{2}{3 \gamma}
$$

(D.2) pode ser escrita da seguinte forma:

$$
E^{3}+\frac{2}{\gamma} E-\frac{2}{\gamma} E_{\text {lin }}=0 .
$$

Logo, usando (D.3)

$$
\left(E^{+}+E^{-}\right)^{3}+\frac{2}{\gamma}\left(E^{+}+E^{-}\right)-\frac{2}{\gamma} E_{\text {lin }}=0 .
$$

Expandimos o termo cúbico, e obtemos:

$$
\left(E^{+}\right)^{3}+\left(E^{-}\right)^{3}+\left(3 E^{+} E^{-}+\frac{2}{\gamma}\right)\left(E^{+}+E^{-}\right)-\frac{2}{\gamma} E_{\operatorname{lin}}=0 .
$$

Pelas equações (D.4) e (D.7), obtemos um sistema de duas equações:

$$
\begin{aligned}
\left(E^{+}\right)^{3}+\left(E^{-}\right)^{3} & =\frac{2}{\gamma} E_{\text {lin }}, \\
\left(E^{+}\right)^{3}\left(E^{-}\right)^{3} & =-\frac{8}{27} \frac{1}{\gamma^{3}} .
\end{aligned}
$$


A segunda equação de (D.8) nos fornece:

$$
\left(E^{\mp}\right)^{3}=-\frac{8}{27} \frac{1}{\gamma^{3}} \frac{1}{\left(E^{ \pm}\right)^{3}}
$$

Pela substituição da primeira equação de (D.8), obtemos uma equação de sexto grau em $E$ :

$$
\left(E^{ \pm}\right)^{6}-\frac{2}{\gamma} E_{\text {lin }}\left(E^{ \pm}\right)^{3}-\frac{8}{27} \frac{1}{\gamma^{3}}=0 .
$$

Esta é uma equação quadrada em $\left(E^{ \pm}\right)^{3}$, que nos fornece duas possíveis soluções:

$$
\left(E^{ \pm}\right)^{3}=\frac{E_{\text {lin }}}{\gamma} \pm \sqrt{\left(\frac{E_{\text {lin }}}{\gamma}\right)^{2}+\left(\frac{2}{3 \gamma}\right)^{3}}
$$

Portanto, tendo em mente que queremos soluções reais para $E$, e observando que o discriminante é sempre positivo, podemos tomar a raiz cúbica real como sendo a única solução:

$$
E^{ \pm}=\sqrt[3]{\frac{E_{\text {lin }}}{\gamma} \pm \sqrt{\left(\frac{E_{\text {lin }}}{\gamma}\right)^{2}+\left(\frac{2}{3 \gamma}\right)^{3}}}
$$

Logo, por (D.3) obtemos a solução analítica para o campo não linear em termos do campo linear:

$$
E(r)=\sqrt[3]{\frac{E_{\operatorname{lin}}(r)}{\gamma}+\sqrt{\left(\frac{E_{\operatorname{lin}}(r)}{\gamma}\right)^{2}+\left(\frac{2}{3 \gamma}\right)^{3}}}+\sqrt[3]{\frac{E_{\operatorname{lin}}(r)}{\gamma}-\sqrt{\left(\frac{E_{\operatorname{lin}}(r)}{\gamma}\right)^{2}+\left(\frac{2}{3 \gamma}\right)^{3}}} .
$$




\section{Referências Bibliográficas}

[1] C. V. Costa, D.M. Gitman, and A.E.Shabad, Phys. Rev. D 88, 085026 (2013).

[2] V. B. Berestetsky, E. M. Lifshitz, and L. P. Pitayevsky, Quantum Electrodynamics (Nauka, Moscow, 1989; Pergamon Press Oxford, New York, 1982).

[3] S. Weinberg, The Quantum Theory of Fields (University Press, Cambridge, 2001).

[4] D. M. Gitman and I. V. Tyutin, Quantization of Fields with Constraints (Springer-Verlag, Berlim, 1990).

[5] F. Sauter, Über das Verhalten eines Elektrons im homogenen elektrischen Feld nach der relativistischen Theorie Diracs, Zeitschrift für Physik, 82 (1931).

[6] D. M. Gitman, A. E. Shabad, Nonlinear (magnetic) correction to the field of a static charge in an external field, Phys. Rev. D, 86, 12 (2012).

[7] B.J. Ahmedov, B.B. Ahmedov, A.A. Abdujabbarov, arXiv:1110.6586 [astro-ph.SR] 338, 157 (2012).

[8] Jerzy Plebański, Lectures on Nonlinear Electrodynamics (Nordita, Copenhagen, 1970).

[9] M. Born and L. Infeld, Proc. Roy. Soc. A, 144, 425 (1934).

[10] A. Stern, Phys. Rev. Lett. 100, 061601 (2008).

[11] T. C. Adorno, D. M. Gitman, A. E. Shabad, D. V. Vassilevich, Phys.Rev. D, 84, 085031 (2011); 84, 065003 (2011); T. C. Adorno, D. M. Gitman, A. E. Shabad, Phys. Rev. D, 86, 027702 (2012).

[12] T. Vachaspati, Phys. Lett. B, 265, 258 (1991); K. Enquist and P. Olesen, Phys. Lett. B, 319, 178 (1993); D. Grasso and H. R. Rubinstein, Phys. Rept. 348, 163 (2001).

[13] R. C. Duncan, C. Thompson, Astro. Phys. J., 392, L9 (1992); J. M. Lattimer, M. Prakash, Phys. Rept., 442, 109 (2007).

[14] A. K. Harding, D. Lai, Physics of strongly magnetized neutron stars, Rep. Prog. Phys. 69, 2631 (2006).

[15] R. P. Negreiros, F. Weber, M. Malheiro, V. Usov, Phys. Rev. D.80, 083006 (2009); R. P. Negreiros, I. N. Mishustin, S. Schramm, F. Weber, Phys. Rev. D, 82, 103010 (2010).

[16] D. E.Kharzeev, L. D. McLerran and H. J. Warringa, Nucl. Phys. A803, 227 (2008); V. S. Skokov, A. Illarionov and V. Toneev, Int. J. Mod. Phys. A24, 5925 (2009). 
[17] J. D. Jackson, Classical Electrodynamics (John Wiley \& Sons, 1962).

[18] G. Arfken, Mathematical methods for physicists (Miami University, Academic Press, Inc., Third edition, 2006).

[19] V. S. Beskin, A. V. Gurevich and Ya. N. Istomin, Physics of the Pulsar Magnetosphere (Cambridge, 1993).

[20] R. Turolla, S. Zane, J. A. Pons, P. Esposito, N. Rea, Is SGR. 0418+5729 indeed a waning magnetar? arXiv:1107.5488 [astro-ph.HE] 740, 105 (2011).

[21] R. X. Xu, $1 E$ 1207.4-5209: a low-mass bare strange star?, arXiv:astro-ph/0402659v23.

[22] V. M. Kaspi, M. E. Gonzalez, F. Camilo, B. M. Gaensler, M. J. Pivovaroff, PSR J1119-6127 and the X-ray Emission from High Magnetic Field Radio Pulsars, arXiv:astro-ph/0610522v2.

[23] F.X. Lee, R. Kelly, L. Zhou, W. Wilcox, Baryon magnetic moments in the background field methods, Phys.Lett. B627, 71 (2005), arXiv: 0509067 [hep-lat]

[24] M.N. Butler, M.J. Savage, and R.P. Springer, Phys. Rev. D 49, 3459 (1994), hep-ph/9308317; L. Geng, arXiv: 1301.7815 [nucl-th]

[25] E. Witten, Phys. Rev. D, 30, 272 (1984).

[26] K. Nakamura et al. [ Particle Data Group Collaboration], J. Phys. G G37, 075021 (2010).

[27] E. S. Fradkin, D. M. Gitman and S. M. Shvartsman, Quantum Electrodynamics with Unstable Vacuum (Springer, Berlin, 1991).

[28] L. P. Linares, M. Malheiro, A. R. Taurines, and M. Fiolhais, Brazilian Journ. Phys. 36, 1391 (2006).

[29] R. P. Negreiros, F. Weber, M. Malheiro, V. Usov, Phys. Rev. D 80, 083006 (2009); R. P. Negreiros, I. N. Mishustin, S. Schramm, F. Weber, Phys. Rev. D 82, 103010 (2010).

[30] T.C. Adorno, D. M. Gitman, A. E. Shabad, Electric charge is a magnetic dipole when placed in a background magnetic field, Phys. Rev. D 86, 125028 (2012).

[31] M. Born and L. Infeld, Nature, 132, 970 (1933); F. Rohrlich, Classical charged particles (World Scientific, 2007).

[32] A.E. Shabad and V.V. Usov, Phys. Rev. Lett. 98, 180403 (2007); arXiv: 0707.3475; Phys. Rev. D 77, 025001 (2008); "String-Like Electrostatic Interaction from QED with Infinite Magnetic Field." in: "Particle Physics on the Eve of LHC"(Proc. of the 13th Lomonosov Conference on Elementary Particle Physics, Moscow, August 2007), Ed. A.I. Studenikin, World Scientific, Singapore, 392 (2009), arXiv:0801.0115 [hep-th].

[33] N. Sadooghi and S. Jalili, Phys. Rev. D 76, 065013 (2007).

[34] B. Machet and M.I. Vysotsky, Phys. Rev. D 83, 025022 (2011); S.I. Godunov, B. Machet, and M. Vysotsky, Phys. Rev. D 85, 044058 (2012).

[35] S. Villalba-Chávez and A.E. Shabad, arXiv:1206.4491. 
[36] A.E. Shabad and V.V. Usov, arXiv: 0991.0640 [hep-th].

[37] A.E. Shabad and V.V. Usov, Phys. Rev. D 83, 105006 (2011).

[38] A.E. Shabad, Polarization of the Vacuum and a Quantum Relativistic Gas in an External Field (Nova Science Publishers, New York, 1991). See also in Polarization effects in external gauge fields. Ed. V.L. Ginzburg, Proc. P.N. Lebedev Phys. Inst. 192, 5 ( Nauka, Moscow, 1988) in Russian).M. Born and L. Infeld, Nature, 132, 970 (1933); F. Rohrlich, Classical charged particles (World Scientific, 2007).

[39] A. I. Milstein, and M. Schumacher, Phys. Rep. 243, 183 (1994); A. Di Piazza, and A. I. Milstein, Phys. Rev. A 77, 042102 (2008); R. N. Lee et al., Phys. Rep. 373, 213 (2003); A. Di Piazza, A. I. Milstein, and C. H. Keitel, Phys. Rev. A 76, 032103 (2007).

[40] A. E. Shabad and V.V. Usov, Phys. Rev. Lett. 98, 180403 (2007); Phys. Rev. D 77, 025001 (2008); arXiv:0801.0115; N. Sadooghi and S. Jalili, Phys. Rev. D 76, 065013 (2007); B. Machet and M.I. Vysotsky, Phys. Rev. D 83, 025022 (2011); S.I. Godunov, B. Machet, and M. Vysotsky, Phys. Rev. D 85, 044058 (2012).

[41] G. Jarlskog et al., Phys. Rev. D 8, 3813 (1973); Sh. Zh. Akhmadaliev et al., Phys. Rev. C 58, 2844 (1998), Phys. Rev. Lett. 89, 061802 (2002).

[42] D. d'Enterria and G. G. Silveira, Phys. Rev. Lett. 111, 080405 (2013).

[43] G. Cardano, Ars magna or The Rules of Algebra (Dover, 1993).

[44] J.M. Dávila, C. Schubert, and M. Anabel Trejo, arXiv:1310.8410 [hep-ph].

[45] S. Weinberg, The Quantum Theory of Fields (University Press, Cambridge, 2001).

[46] D. M. Gitman and A. E. Shabad, Phys. Rev. D 86, 125028 (2012); T.C. Adorno, D. M. Gitman, and A. E. Shabad, arXiv:1311.4081[hep-th].

[47] H.A. Lorentz, Weiterbildung der Maxwell'schen Theorie: Elektronentheorie, Encyklopädie d. Mathematischen Wissenschaften, Band V2, Heft 1, Art. 14, 145-288 (1904); Abraham, M., Theorie der Elektrizität, II (Teubner, Leipzig 1905,1923); Spohn, H., Dynamics of charged particles and their radiation field (Cambridge University Press, Cambridge, 2004).

[48] L. Infeld, Nature 137, 658 (1936).

[49] S.M. Blinder, arXiv:physics/0208072. 